\title{
Grayanane Diterpenoids from the Leaves of Rhododendron auriculatum and Their Analgesic Activities
}

Na Sun, ${ }^{\dagger}$ Guijuan Zheng, ${ }^{\dagger}$ Meijun He, ${ }^{\dagger}$ Yuanyuan Feng, ${ }^{\dagger}$ Junjun Liu, ${ }^{\dagger}$ Meicheng Wang, ${ }^{\dagger}$ Hanqi Zhang, ${ }^{\dagger}$ Junfei Zhou, ${ }^{\dagger}$ and Guangmin Yao ${ }^{*} \dagger$

${ }^{\dagger}$ Hubei Key Laboratory of Natural Medicinal Chemistry and Resource Evaluation, School of Pharmacy, Tongji Medical College, Huazhong University of Science and Technology, Wuhan 430030, People's Republic of China

Institute of Chinese Herbal Medicines, Hubei Academy of Agricultural Sciences, Enshi 445500, People's Republic of China

\section{List of Supporting Information}

Figure S1. ${ }^{1} \mathrm{H}-{ }^{1} \mathrm{H}$ COSY, key HMBC, and NOESY correlations of compound 2...........................6

Figure S2. ${ }^{1} \mathrm{H}-{ }^{1} \mathrm{H}$ COSY, key HMBC, and NOESY correlations of compound 3...........................6

Figure S3. ${ }^{1} \mathrm{H}-{ }^{1} \mathrm{H}$ COSY, key HMBC, and NOESY correlations of compound 4..........................6

Figure S4. ${ }^{1} \mathrm{H}-{ }^{1} \mathrm{H}$ COSY, key HMBC, and NOESY correlations of compound 5..........................6

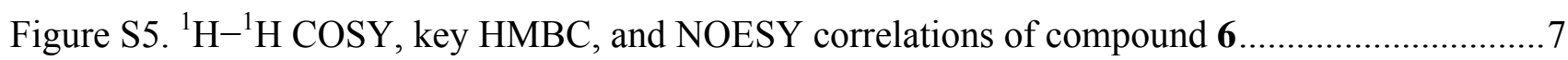

Figure S6. ${ }^{1} \mathrm{H}-{ }^{1} \mathrm{H}$ COSY, key HMBC, and NOESY correlations of compound 7...........................7

Figure S7. ${ }^{1} \mathrm{H}-{ }^{1} \mathrm{H}$ COSY, key HMBC, and NOESY correlations of compound 8...........................7

Figure S8. ${ }^{1} \mathrm{H}-{ }^{1} \mathrm{H}$ COSY, key HMBC, and NOESY correlations of compound 9...........................7

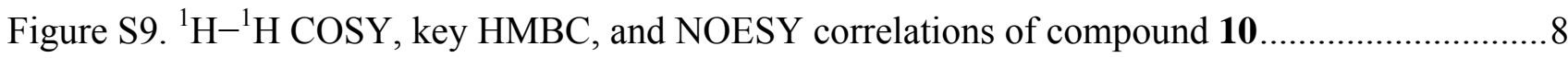

Figure S10. ${ }^{1} \mathrm{H}^{-1} \mathrm{H}$ COSY, key HMBC, and NOESY correlations of compound 11....................... 8

Figure S1 $1 .{ }^{1} \mathrm{H}^{-1} \mathrm{H}$ COSY, key HMBC, and NOESY correlations of compound 12....................... 8

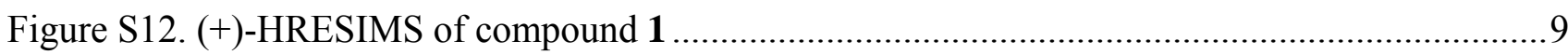

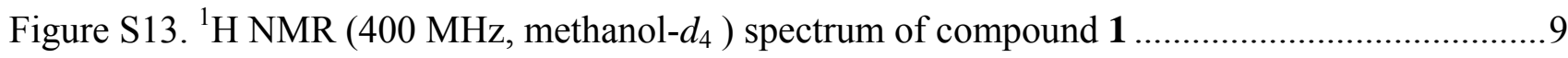

Figure S14. ${ }^{13} \mathrm{C}$ NMR (100 MHz, methanol- $\left.d_{4}\right)$ spectrum of compound 1 .................................... 10

\footnotetext{
*To whom correspondence should be addressed.

E-mail: gyap@mail.hust.edu.cn
} 
Figure S15. DEPT (100 MHz, methanol- $\left.d_{4}\right)$ spectrum of compound 1 10

Figure S16. HSQC (methanol- $\left.d_{4}\right)$ spectrum of compound $1\left({ }^{1} \mathrm{H}: 400 \mathrm{MHz},{ }^{13} \mathrm{C}: 100 \mathrm{MHz}\right) \ldots \ldots \ldots \ldots . .11$

Figure S17. HMBC (methanol- $d_{4}$ ) spectrum of compound $1\left({ }^{1} \mathrm{H}: 400 \mathrm{MHz},{ }^{13} \mathrm{C}: 100 \mathrm{MHz}\right) \ldots \ldots \ldots . .11$

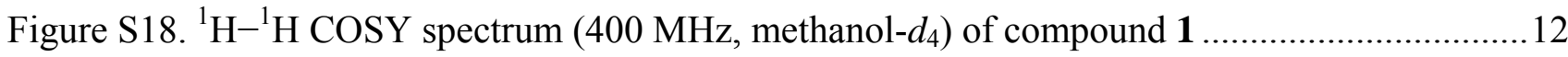

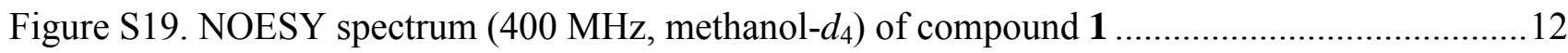

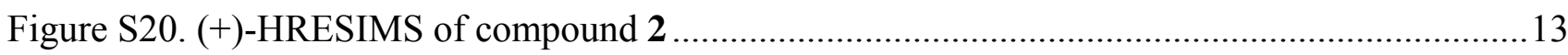

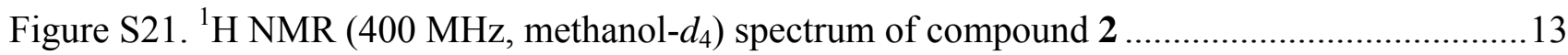

Figure S22. ${ }^{13} \mathrm{C}$ NMR (100 MHz, methanol- $\left.d_{4}\right)$ spectrum of compound 2 ...................................... 14

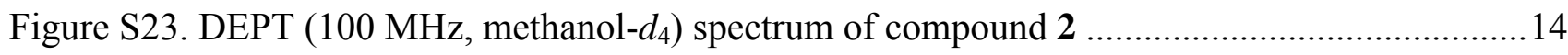

Figure S24. HSQC (methanol- $\left.d_{4}\right)$ spectrum of compound $2\left({ }^{1} \mathrm{H}: 400 \mathrm{MHz},{ }^{13} \mathrm{C}: 100 \mathrm{MHz}\right) \ldots \ldots \ldots \ldots . . . . .15$

Figure S25. HMBC (methanol- $d_{4}$ ) spectrum of compound $2\left({ }^{1} \mathrm{H}: 400 \mathrm{MHz},{ }^{13} \mathrm{C}: 100 \mathrm{MHz}\right) \ldots \ldots \ldots . .15$

Figure S26. ${ }^{1} \mathrm{H}-{ }^{1} \mathrm{H}$ COSY (400 MHz, methanol- $d_{4}$ ) spectrum of compound 2 ............................... 16

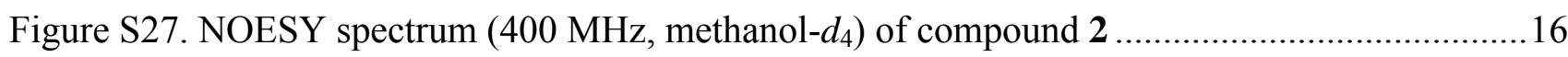

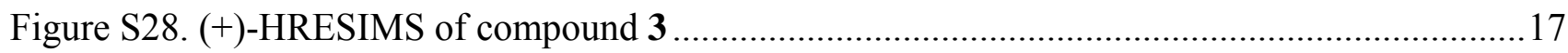

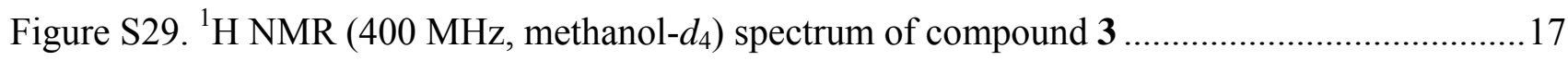

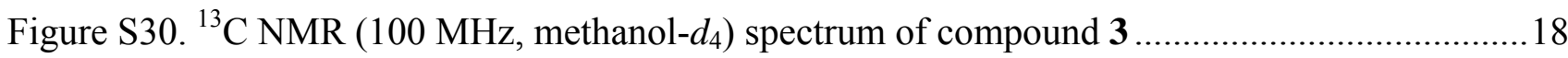

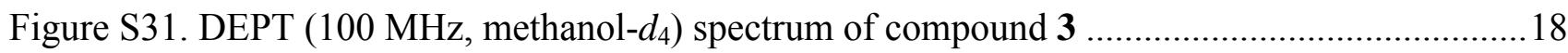

Figure S32. HSQC (methanol-d $\left.d_{4}\right)$ spectrum of compound $3\left({ }^{1} \mathrm{H}: 400 \mathrm{MHz},{ }^{13} \mathrm{C}: 100 \mathrm{MHz}\right) \ldots \ldots \ldots \ldots 19$

Figure S33. HMBC (methanol- $\left.d_{4}\right)$ spectrum of compound $3\left({ }^{1} \mathrm{H}: 400 \mathrm{MHz},{ }^{13} \mathrm{C}: 100 \mathrm{MHz}\right) \ldots \ldots \ldots \ldots . . .19$

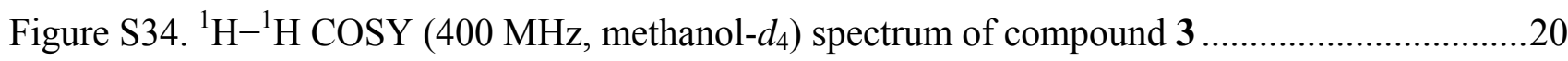

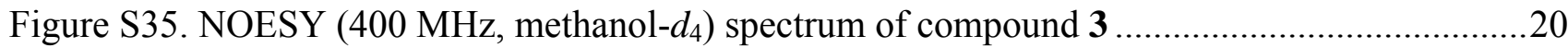

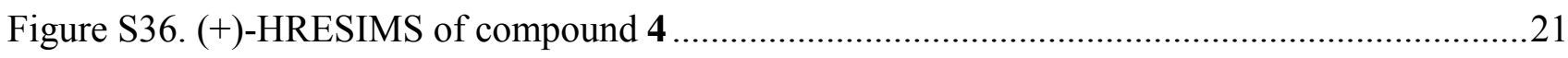

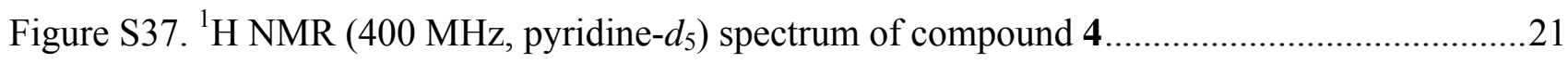

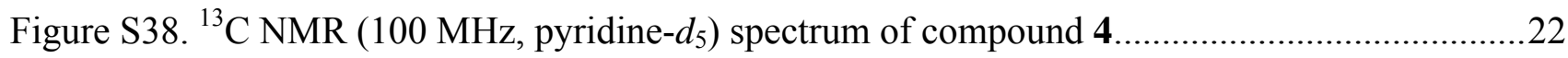

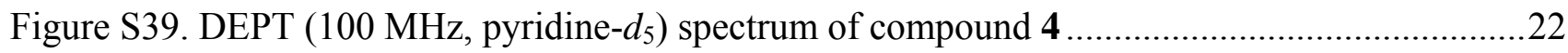

Figure S40. HSQC (pyridine- $d_{5}$ ) spectrum of compound $4\left({ }^{1} \mathrm{H}: 400 \mathrm{MHz},{ }^{13} \mathrm{C}: 100 \mathrm{MHz}\right) \ldots \ldots \ldots \ldots . . .23$

Figure S41. HMBC (pyridine- $d_{5}$ ) spectrum of compound $4\left({ }^{1} \mathrm{H}: 400 \mathrm{MHz},{ }^{13} \mathrm{C}: 100 \mathrm{MHz}\right) \ldots \ldots \ldots \ldots . .23$

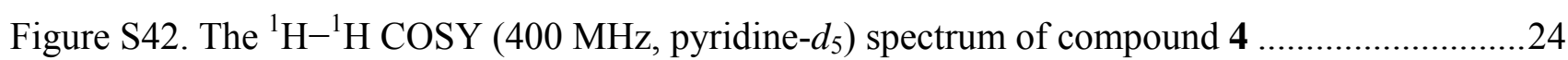

Figure S43. The NOESY (400 MHz, pyridine- $d_{5}$ ) spectrum of compound 4 ...............................24

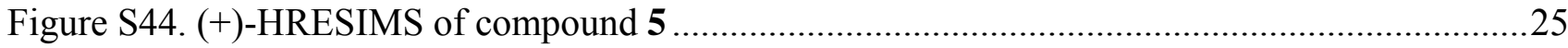




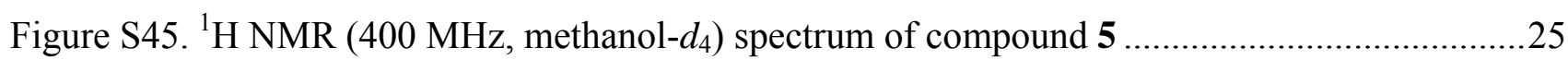

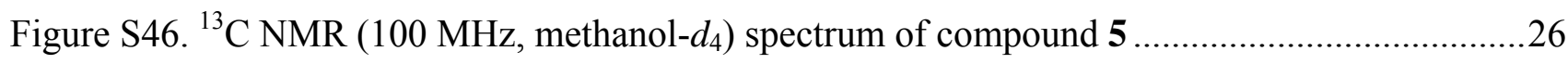

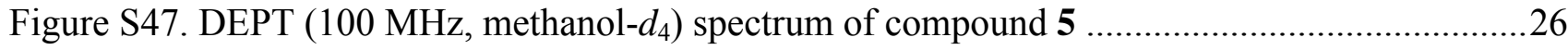

Figure S48. HSQC (methanol- $\left.d_{4}\right)$ spectrum of compound $5\left({ }^{1} \mathrm{H}: 400 \mathrm{MHz},{ }^{13} \mathrm{C}: 100 \mathrm{MHz}\right) \ldots \ldots \ldots \ldots . .27$

Figure S49. HMBC (methanol- $\left.d_{4}\right)$ spectrum of compound $5\left({ }^{1} \mathrm{H}: 400 \mathrm{MHz},{ }^{13} \mathrm{C}: 100 \mathrm{MHz}\right) \ldots \ldots \ldots . .27$

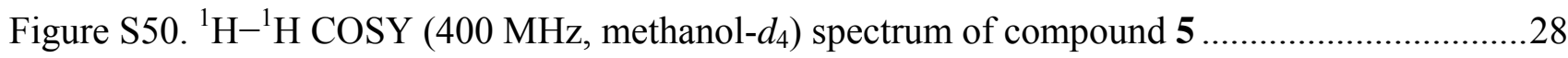

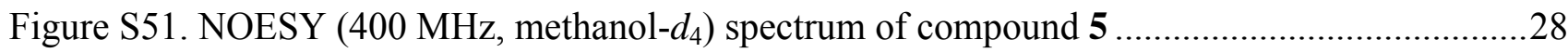

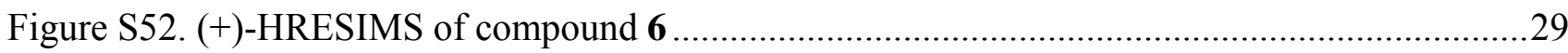

Figure S53. ${ }^{1} \mathrm{H}$ NMR $\left(400 \mathrm{MHz}\right.$, methanol- $\left.d_{4}\right)$ spectrum of compound 6 .....................................29

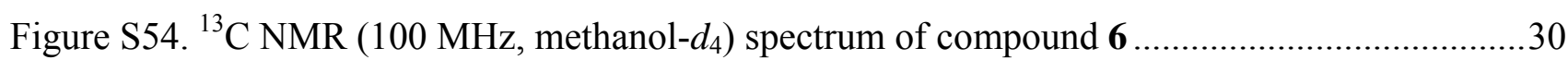

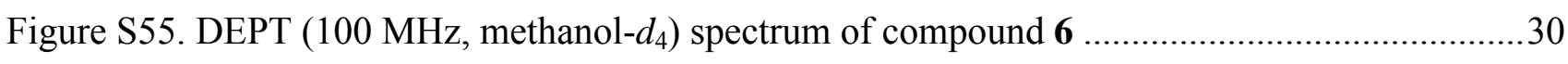

Figure S56. HSQC (methanol- $\left.d_{4}\right)$ spectrum of compound $6\left({ }^{1} \mathrm{H}: 400 \mathrm{MHz},{ }^{13} \mathrm{C}: 100 \mathrm{MHz}\right) \ldots \ldots \ldots . . . .31$

Figure S57. HMBC (methanol- $\left.d_{4}\right)$ spectrum of compound $6\left({ }^{1} \mathrm{H}: 400 \mathrm{MHz},{ }^{13} \mathrm{C}: 100 \mathrm{MHz}\right) \ldots \ldots \ldots . . .31$

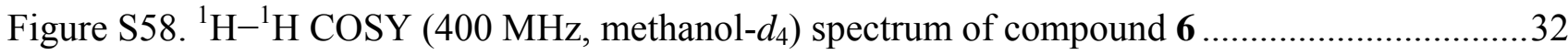

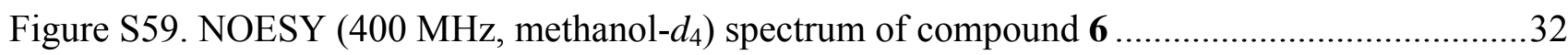

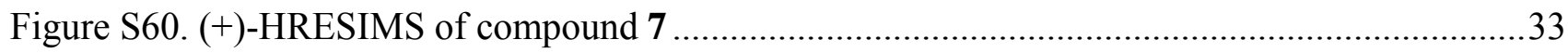

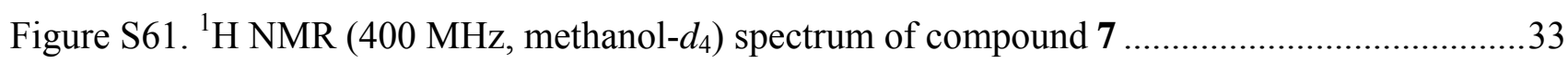

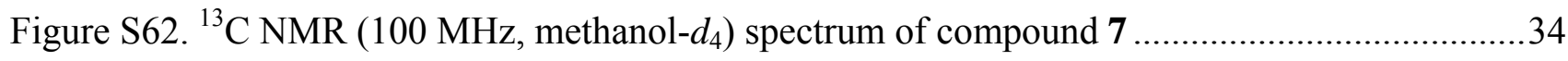

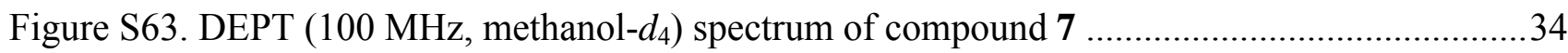

Figure S64. HSQC (methanol- $\left.d_{4}\right)$ spectrum of compound $7\left({ }^{1} \mathrm{H}: 400 \mathrm{MHz},{ }^{13} \mathrm{C}: 100 \mathrm{MHz}\right) \ldots \ldots \ldots \ldots . . . . .35$

Figure S65. HMBC (methanol- $\left.d_{4}\right)$ spectrum of compound $7\left({ }^{1} \mathrm{H}: 400 \mathrm{MHz},{ }^{13} \mathrm{C}: 100 \mathrm{MHz}\right) \ldots \ldots \ldots \ldots . . . .35$

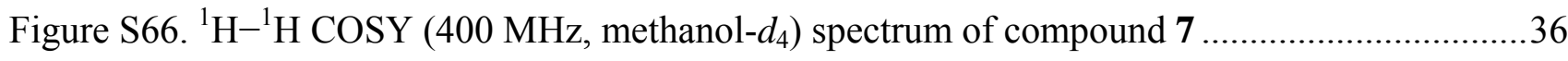

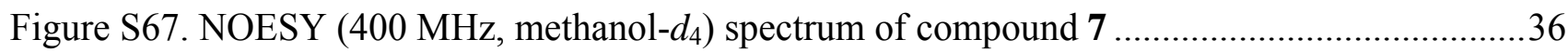

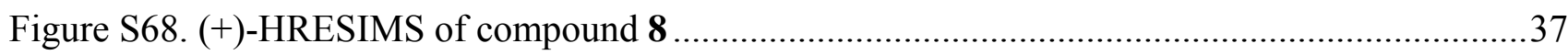

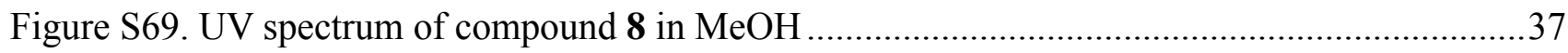

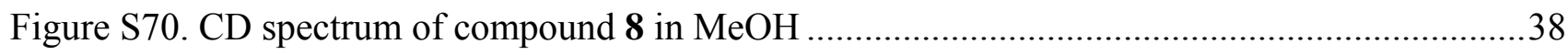

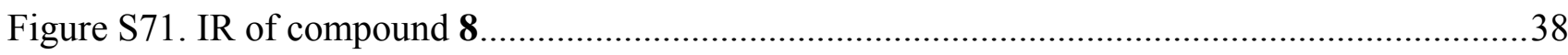

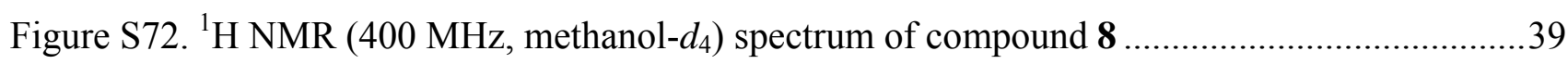

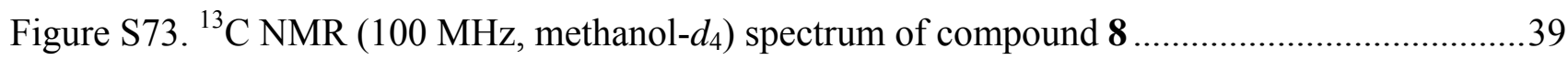

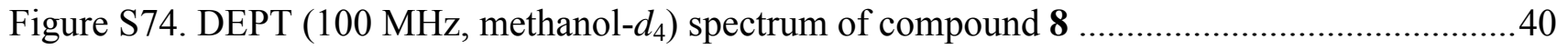


Figure S75. HSQC (methanol- $\left.d_{4}\right)$ spectrum of compound $8\left({ }^{1} \mathrm{H}: 400 \mathrm{MHz},{ }^{13} \mathrm{C}: 100 \mathrm{MHz}\right) \ldots \ldots \ldots \ldots . . . .40$

Figure S76. HMBC (methanol- $\left.d_{4}\right)$ spectrum of compound $8\left({ }^{1} \mathrm{H}: 400 \mathrm{MHz},{ }^{13} \mathrm{C}: 100 \mathrm{MHz}\right) \ldots \ldots \ldots . . .41$

Figure S77. ${ }^{1} \mathrm{H}-{ }^{1} \mathrm{H}$ COSY (400 MHz, methanol-d4) spectrum of compound 8 ............................... 41

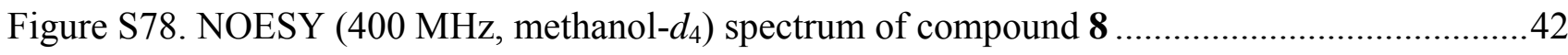

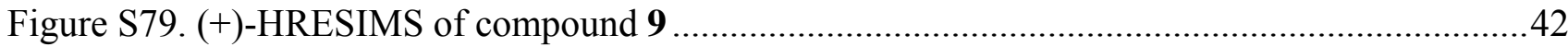

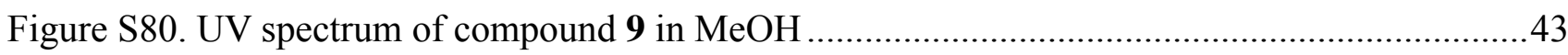

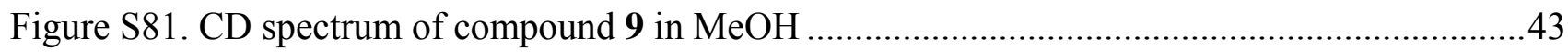

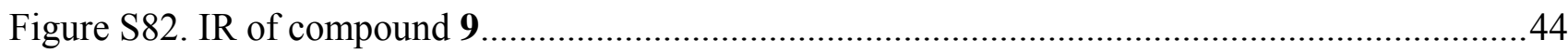

Figure S83. ${ }^{1} \mathrm{H}$ NMR (400 MHz, methanol- $\left.d_{4}\right)$ spectrum of compound 9 .....................................4

Figure S84. ${ }^{13} \mathrm{C}$ NMR (100 MHz, methanol- $\left.d_{4}\right)$ spectrum of compound 9 ......................................45

Figure S85. DEPT (100 MHz, methanol- $\left.d_{4}\right)$ spectrum of compound 9 ........................................45

Figure S86. HSQC (methanol- $\left.d_{4}\right)$ spectrum of compound $9\left({ }^{1} \mathrm{H}: 400 \mathrm{MHz},{ }^{13} \mathrm{C}: 100 \mathrm{MHz}\right) \ldots \ldots \ldots \ldots . . . .46$

Figure S87. HMBC (methanol- $\left.d_{4}\right)$ spectrum of compound $9\left({ }^{1} \mathrm{H}: 400 \mathrm{MHz},{ }^{13} \mathrm{C}: 100 \mathrm{MHz}\right) \ldots \ldots \ldots . . .46$

Figure S88. The ${ }^{1} \mathrm{H}-{ }^{1} \mathrm{H}$ COSY (400 MHz, methanol- $\left.d_{4}\right)$ spectrum of compound $\mathbf{9} \ldots \ldots \ldots \ldots \ldots \ldots \ldots \ldots . . . . . . . . . . . . .47$

Figure S89. The NOESY (400 MHz, methanol- $\left.d_{4}\right)$ spectrum of compound 9 ...............................47

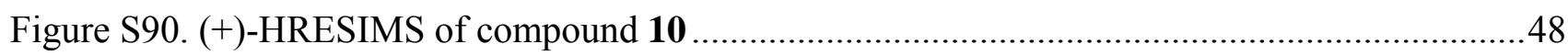

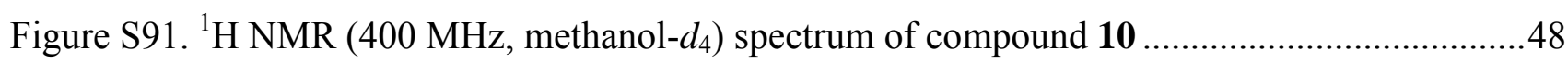

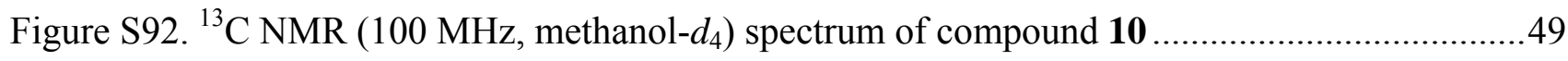

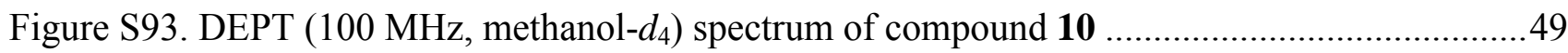

Figure S94. HSQC (methanol-d $\left.d_{4}\right)$ spectrum of compound $10\left({ }^{1} \mathrm{H}: 400 \mathrm{MHz},{ }^{13} \mathrm{C}: 100 \mathrm{MHz}\right) \ldots \ldots \ldots . . . .50$

Figure S95. HMBC (methanol- $\left.d_{4}\right)$ spectrum of compound $10\left({ }^{1} \mathrm{H}: 400 \mathrm{MHz},{ }^{13} \mathrm{C}: 100 \mathrm{MHz}\right) \ldots \ldots \ldots . . .50$

Figure S96. The ${ }^{1} \mathrm{H}-{ }^{1} \mathrm{H}$ COSY (400 MHz, methanol- $\left.d_{4}\right)$ spectrum of compound $\mathbf{1 0} \ldots \ldots \ldots \ldots \ldots \ldots \ldots . . . . . . . . . . .51$

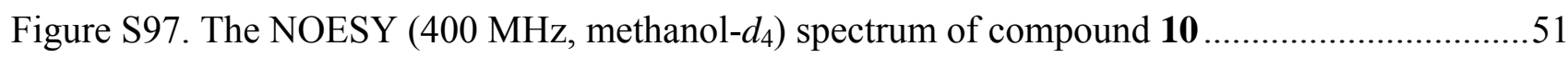

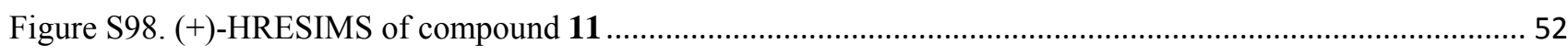

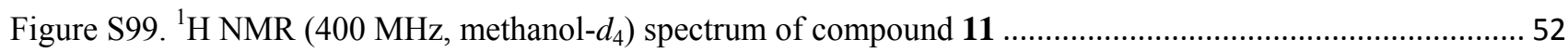

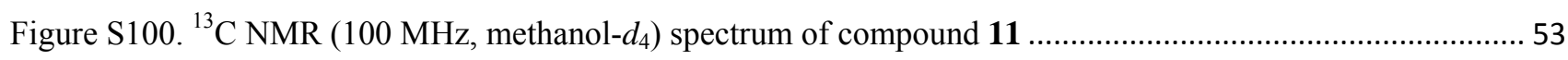

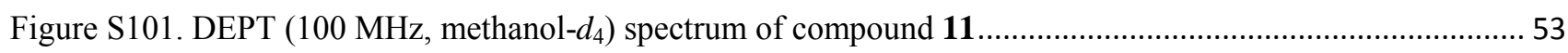

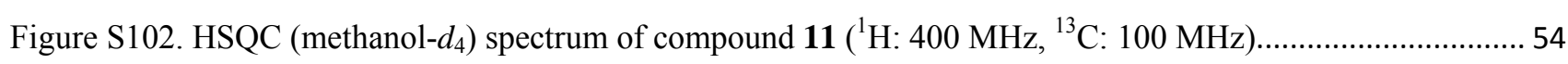

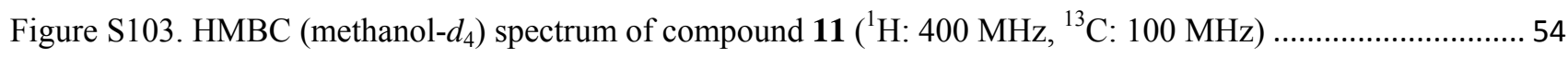

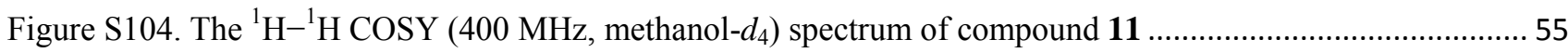


Figure S105. The NOESY (400 MHz, methanol-d $\left.d_{4}\right)$ spectrum of compound 11 55

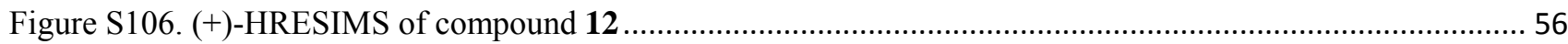

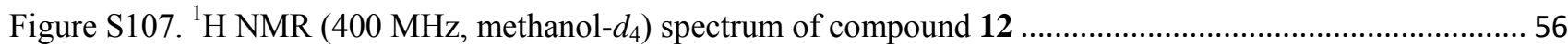

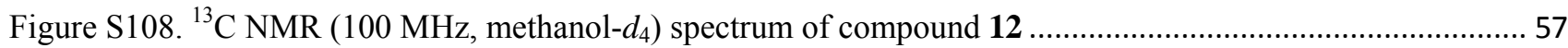

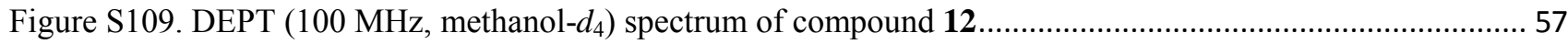

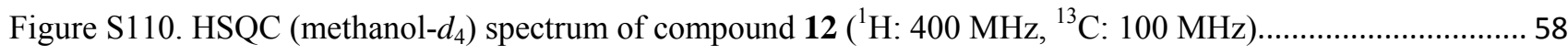

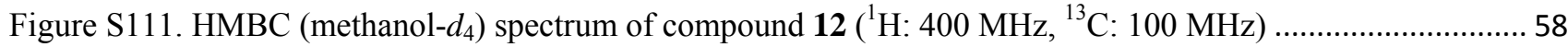

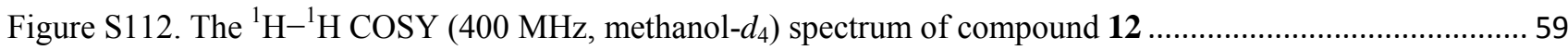

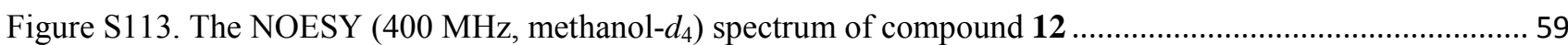



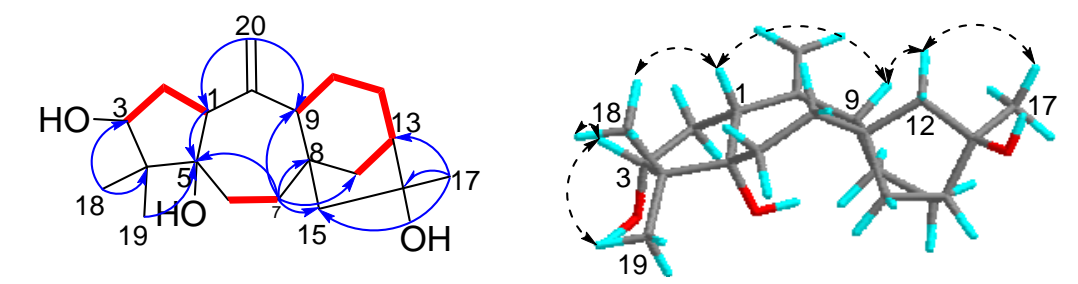

${ }^{1} \mathrm{H}^{1}{ }^{\mathrm{H}}$ COSY: - HMBC: $\overparen{\mathrm{H} \mathrm{C}}$

NOESY: $\mathrm{H}^{-\cdots}$

Figure S1. ${ }^{1} \mathrm{H}-{ }^{1} \mathrm{H}$ COSY, key HMBC, and NOESY correlations of compound 2

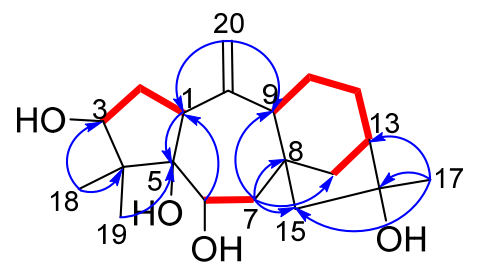

${ }^{1} \mathrm{H}^{-1} \mathrm{H}$ COSY: $-\mathrm{HMBC}: \overparen{\mathrm{HC}}$

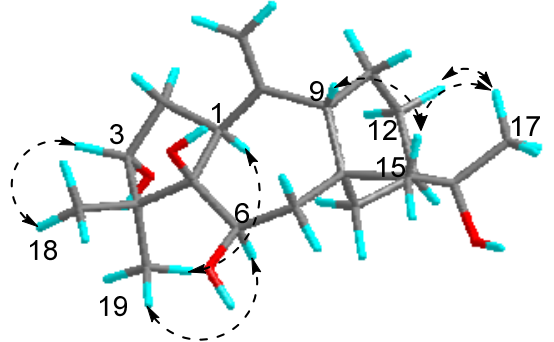

NOESY: $\mathrm{H}^{-\cdots} \mathrm{H}$

Figure S2. ${ }^{1} \mathrm{H}-{ }^{1} \mathrm{H}$ COSY, key HMBC, and NOESY correlations of compound 3

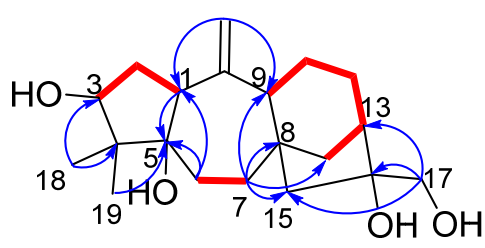

${ }^{1} \mathrm{H}-{ }^{1} \mathrm{H}$ COSY: - HMBC: $\overparen{\mathrm{HC}}$

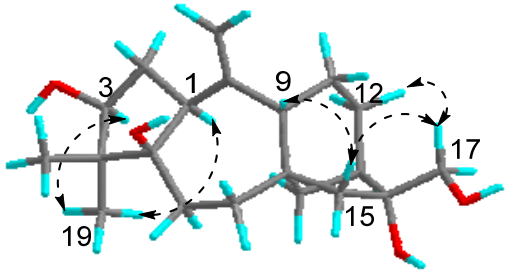

NOESY: $\mathrm{H}^{--} \mathrm{H}$

Figure $\mathrm{S} 3 .{ }^{1} \mathrm{H}-{ }^{1} \mathrm{H}$ COSY, key HMBC, and NOESY correlations of compound 4

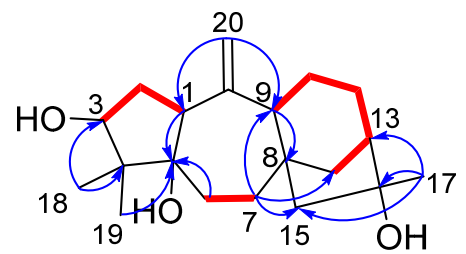

${ }^{1} \mathrm{H}-{ }^{1} \mathrm{H}$ COSY: - HMBC: $\overparen{\mathrm{HC}}$

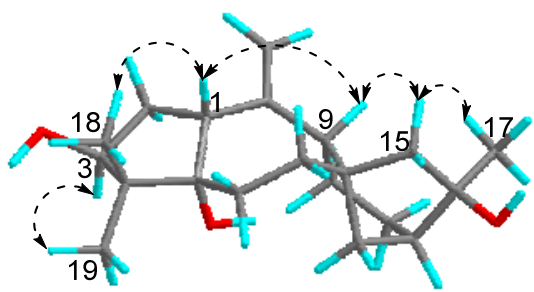

NOESY: $\mathrm{H}^{-\cdots} \mathrm{H}$

Figure S4. ${ }^{1} \mathrm{H}-{ }^{1} \mathrm{H}$ COSY, key HMBC, and NOESY correlations of compound 5 


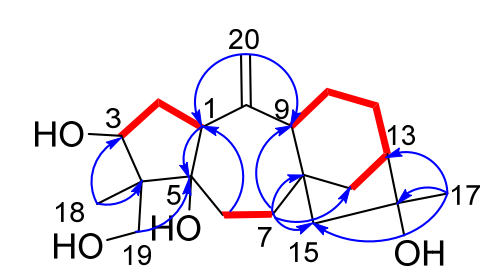

${ }^{1} \mathrm{H}-{ }^{1} \mathrm{H}$ COSY: $-\mathrm{HMBC}: \overparen{\mathrm{HC}}$

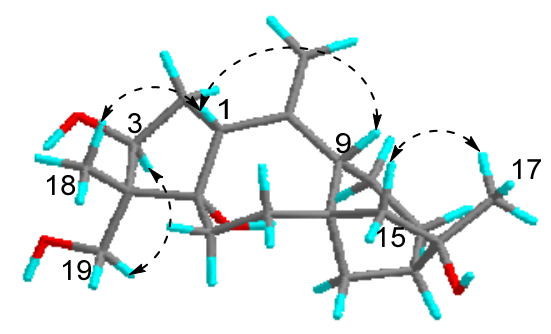

NOESY: $\mathrm{H}^{--} \mathrm{H}$

Figure S5. ${ }^{1} \mathrm{H}-{ }^{1} \mathrm{H}$ COSY, key HMBC, and NOESY correlations of compound 6

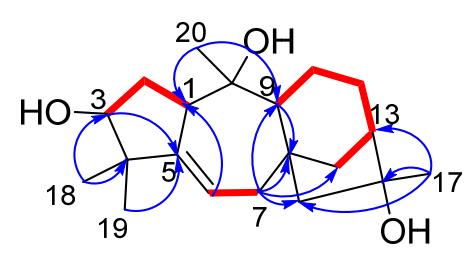

${ }^{1} \mathrm{H}-{ }^{1} \mathrm{H}$ COSY: $-\mathrm{HMBC}: \overparen{\mathrm{HC}}$

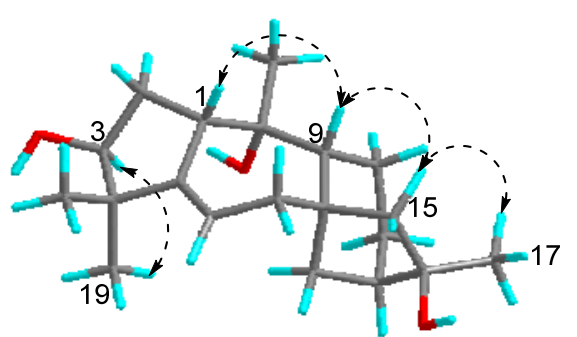

NOESY: $\mathrm{H}^{--} \mathrm{H}$

Figure S6. ${ }^{1} \mathrm{H}-{ }^{1} \mathrm{H}$ COSY, key HMBC, and NOESY correlations of compound 7

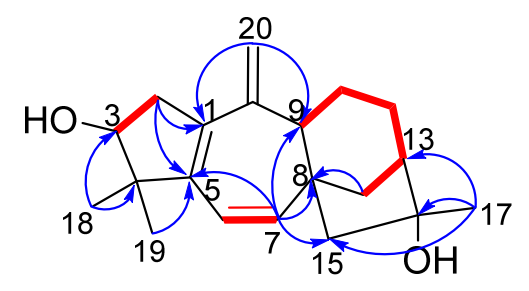

${ }^{1} \mathrm{H}-{ }^{1} \mathrm{H}$ COSY: - HMBC: $\overparen{\mathrm{HC}}$

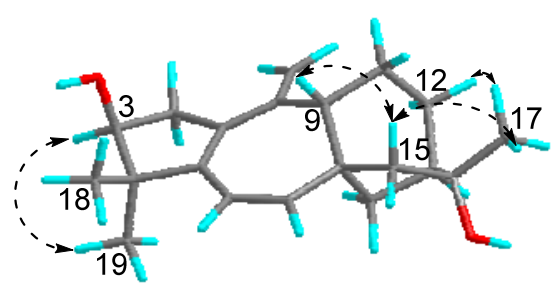

NOESY: $\mathrm{H}^{--} \mathrm{H}$

Figure S7. ${ }^{1} \mathrm{H}^{-1} \mathrm{H}$ COSY, key HMBC, and NOESY correlations of compound 8

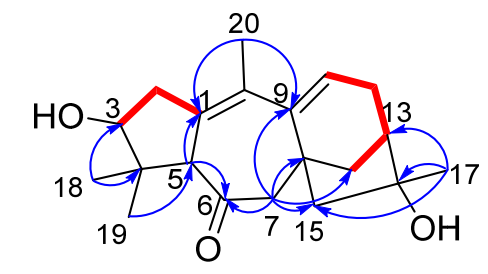

${ }^{1} \mathrm{H}^{-1} \mathrm{H}$ COSY: $-\mathrm{HMBC}: \overparen{\mathrm{HC}}$

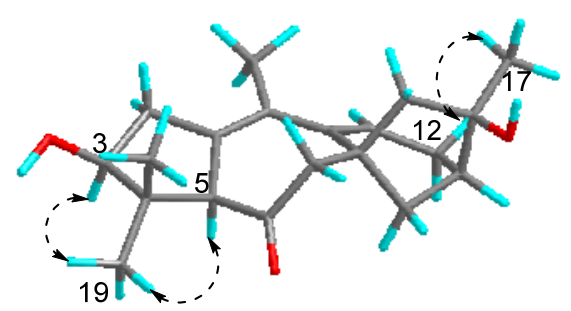

NOESY: $\mathrm{H}^{-\cdots} \mathrm{H}$

Figure S8. ${ }^{1} \mathrm{H}-{ }^{1} \mathrm{H}$ COSY, key HMBC, and NOESY correlations of compound 9 

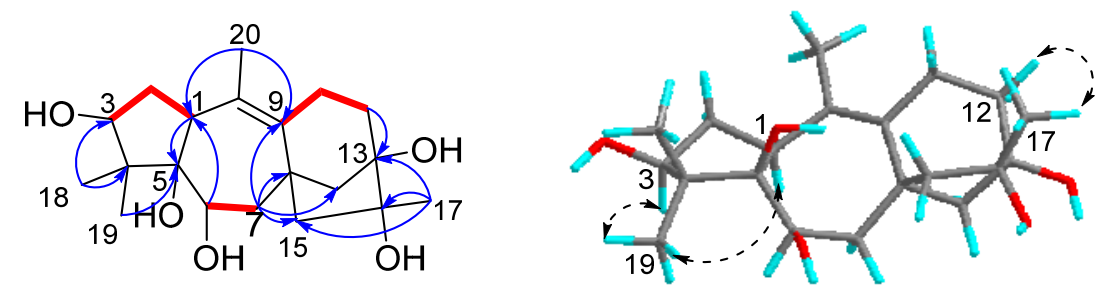

${ }^{1} \mathrm{H}-{ }^{1} \mathrm{H}$ COSY: - HMBC: $\overparen{\mathrm{HC}}$

NOESY: $\mathrm{H}^{-\cdots} \mathrm{H}$

Figure S9. ${ }^{1} \mathrm{H}-{ }^{1} \mathrm{H}$ COSY, key HMBC, and NOESY correlations of compound $\mathbf{1 0}$

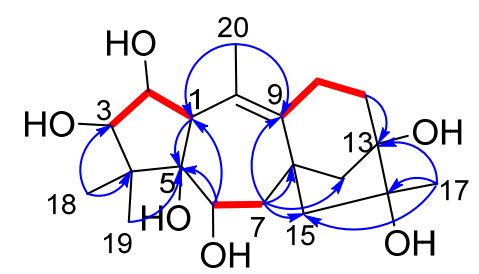

${ }^{1} \mathrm{H}-{ }^{1} \mathrm{H}$ COSY: - HMBC: $\overparen{\mathrm{HC}}$

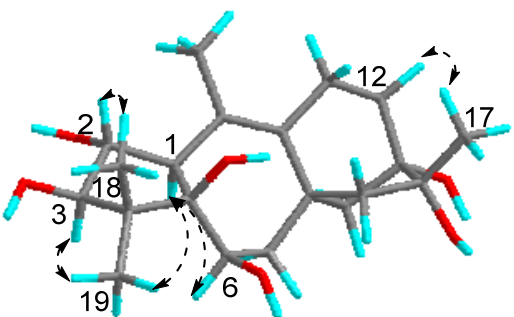

NOESY: H $^{-\cdots}$ Hे

Figure S10. ${ }^{1} \mathrm{H}^{-1} \mathrm{H}$ COSY, key HMBC, and NOESY correlations of compound 11

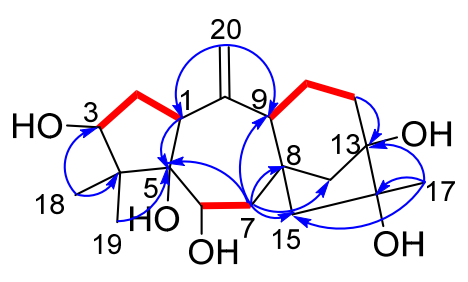

${ }^{1} \mathrm{H}-{ }^{1} \mathrm{H}$ COSY: $-\mathrm{HMBC}: \overparen{\mathrm{HC}}$

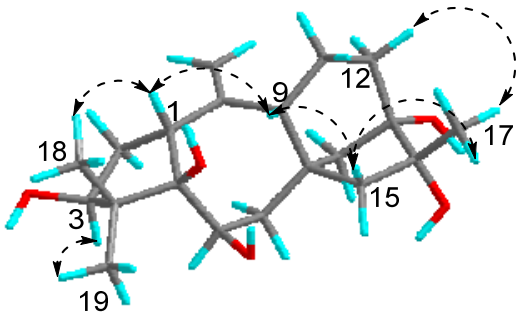

NOESY: $\mathrm{H}^{--} \mathrm{H}$

Figure S11. ${ }^{1} \mathrm{H}-{ }^{1} \mathrm{H}$ COSY, key HMBC, and NOESY correlations of compound 12 


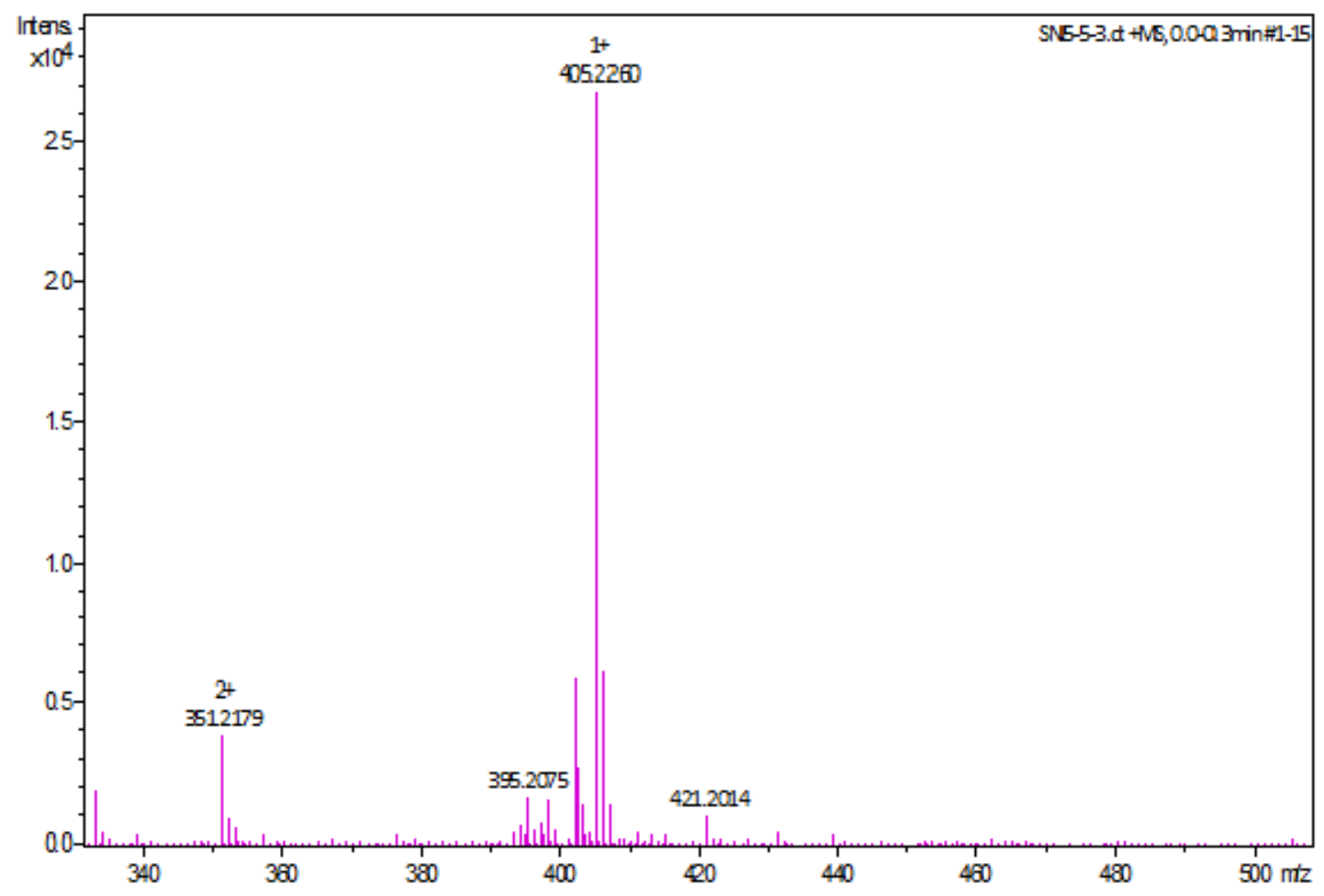

Figure S12. (+)-HRESIMS of compound 1
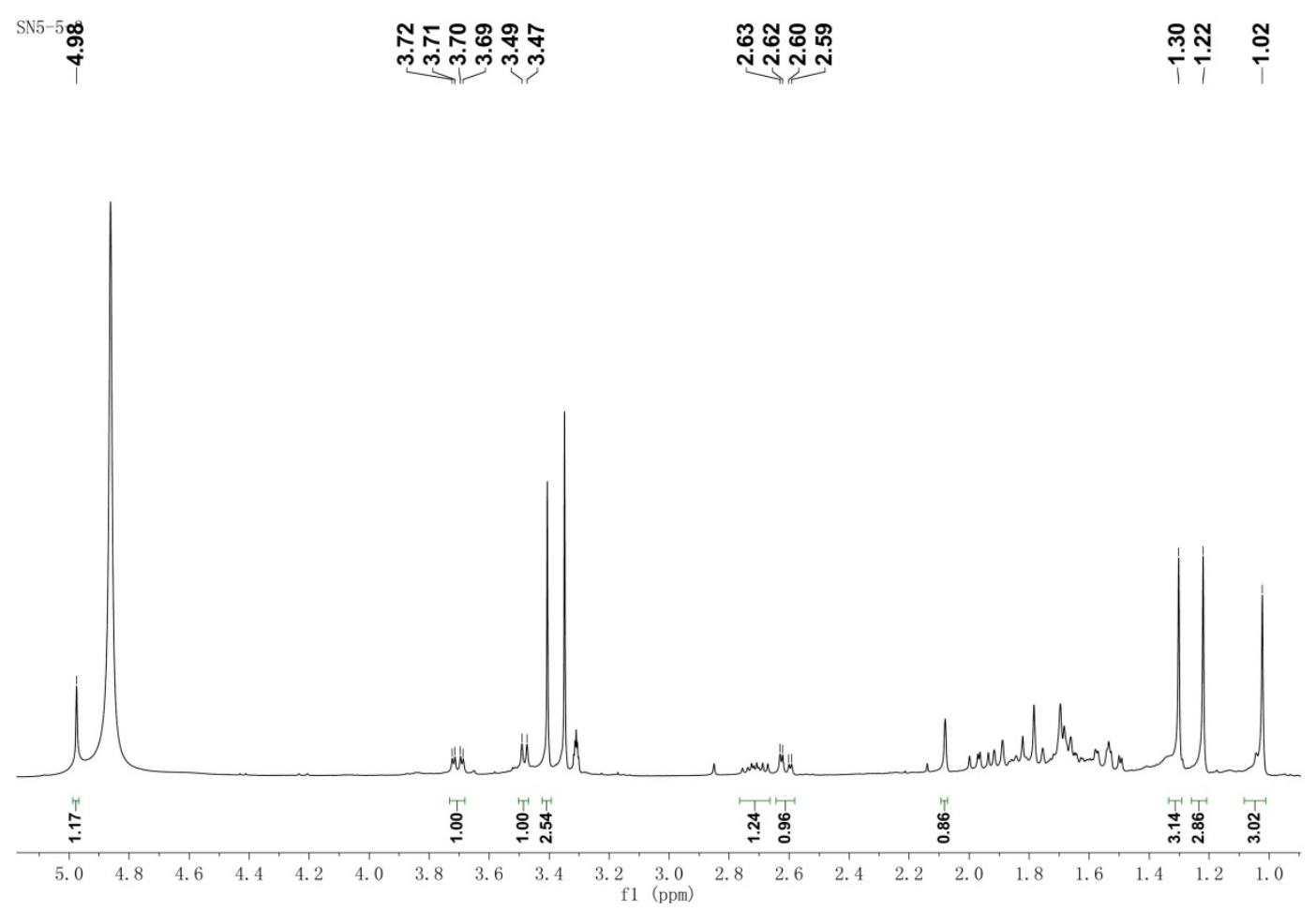

Figure S13. ${ }^{1} \mathrm{H}$ NMR (400 MHz, methanol- $d_{4}$ ) spectrum of compound 1 


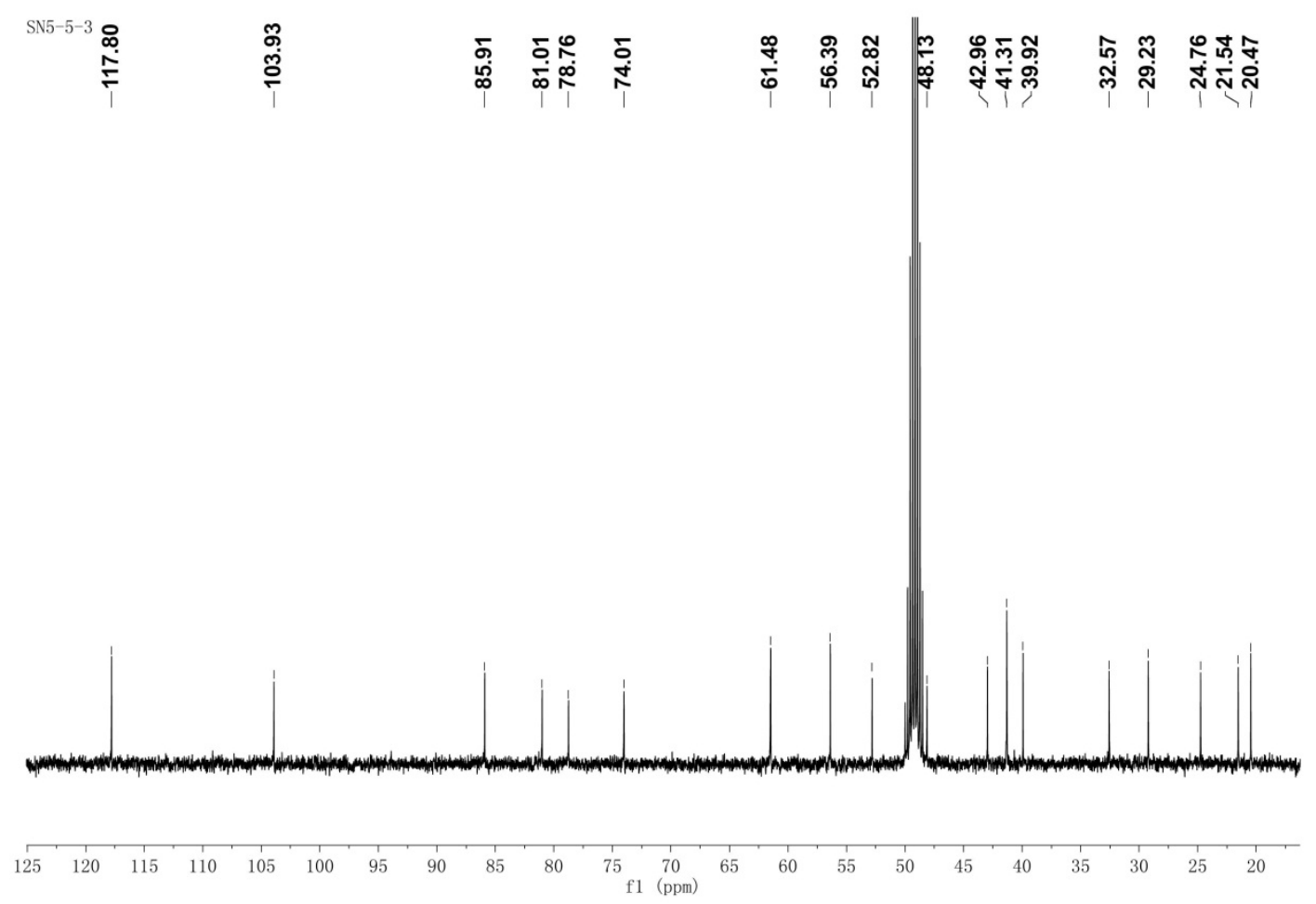

Figure S14. ${ }^{13} \mathrm{C}$ NMR (100 MHz, methanol- $\left.d_{4}\right)$ spectrum of compound 1
$\stackrel{\text { SN5-5 }}{\stackrel{5}{\stackrel{1}{\kappa}}}$
万.
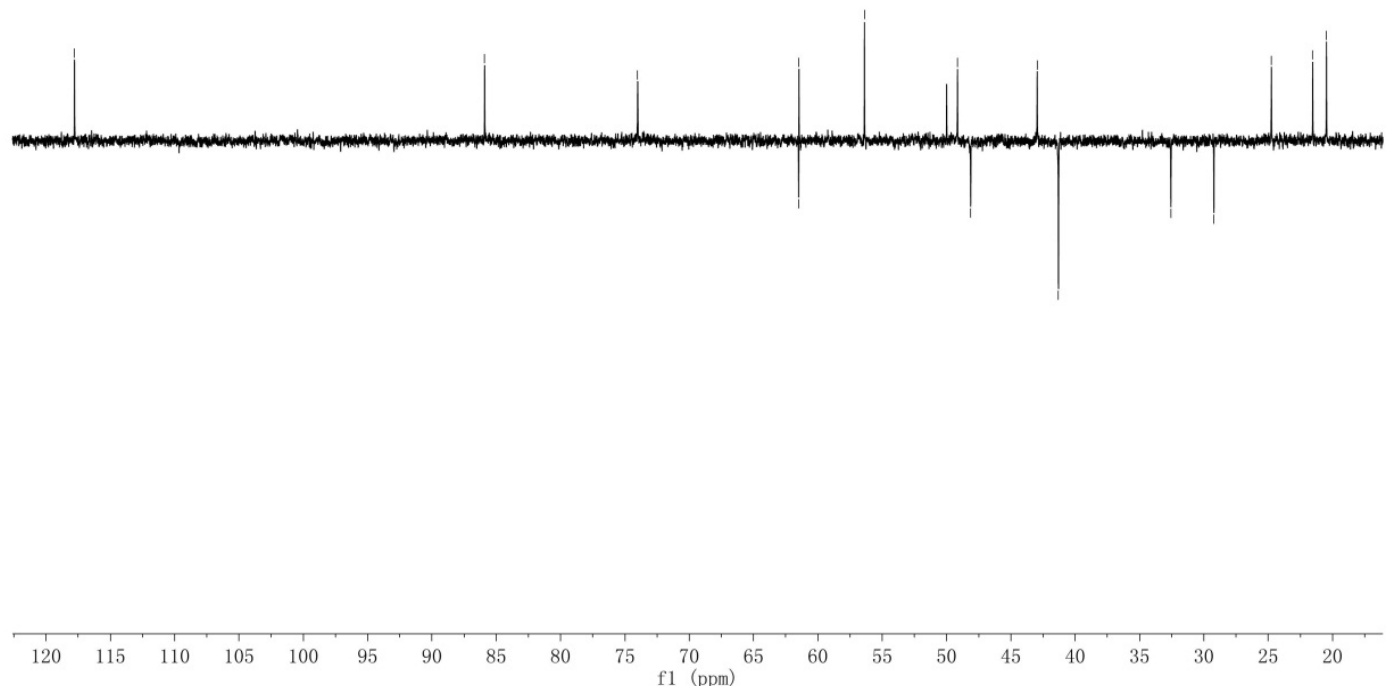

Figure S15. DEPT (100 MHz, methanol- $\left.d_{4}\right)$ spectrum of compound 1 


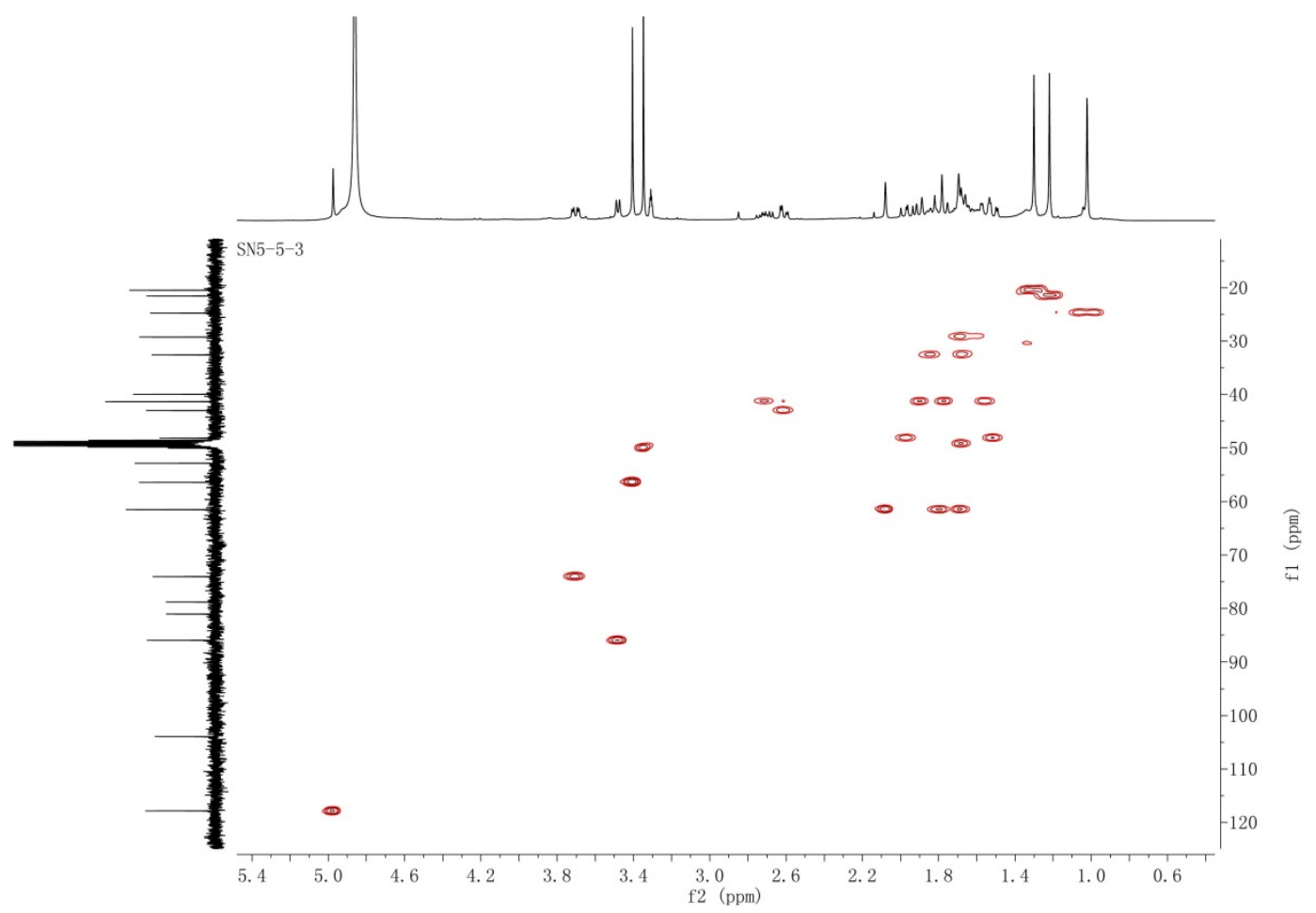

Figure S16. HSQC (methanol- $\left.d_{4}\right)$ spectrum of compound $1\left({ }^{1} \mathrm{H}: 400 \mathrm{MHz},{ }^{13} \mathrm{C}: 100 \mathrm{MHz}\right)$

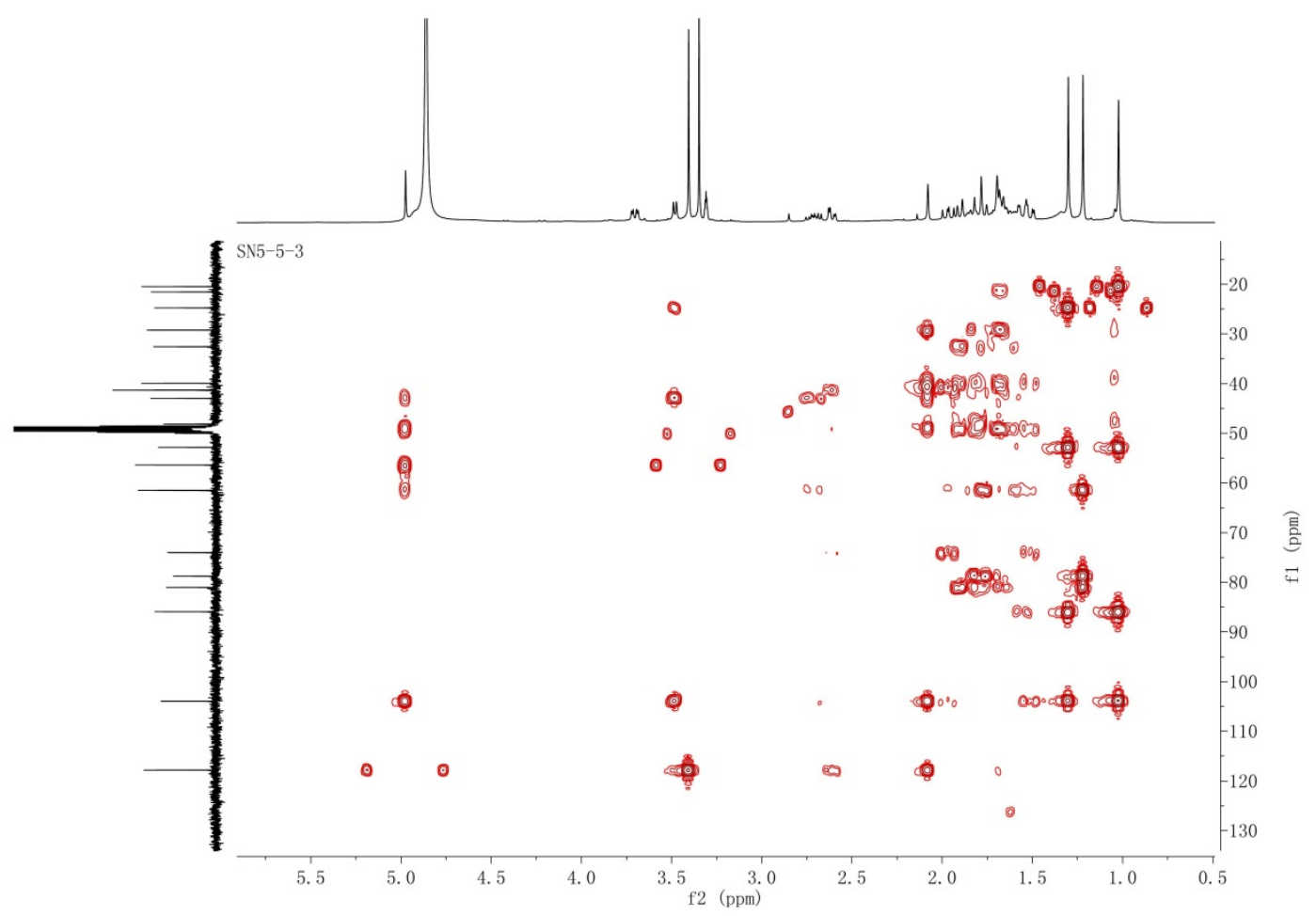

Figure S17. HMBC (methanol- $\left.d_{4}\right)$ spectrum of compound $\mathbf{1}\left({ }^{1} \mathrm{H}: 400 \mathrm{MHz},{ }^{13} \mathrm{C}: 100 \mathrm{MHz}\right)$ 


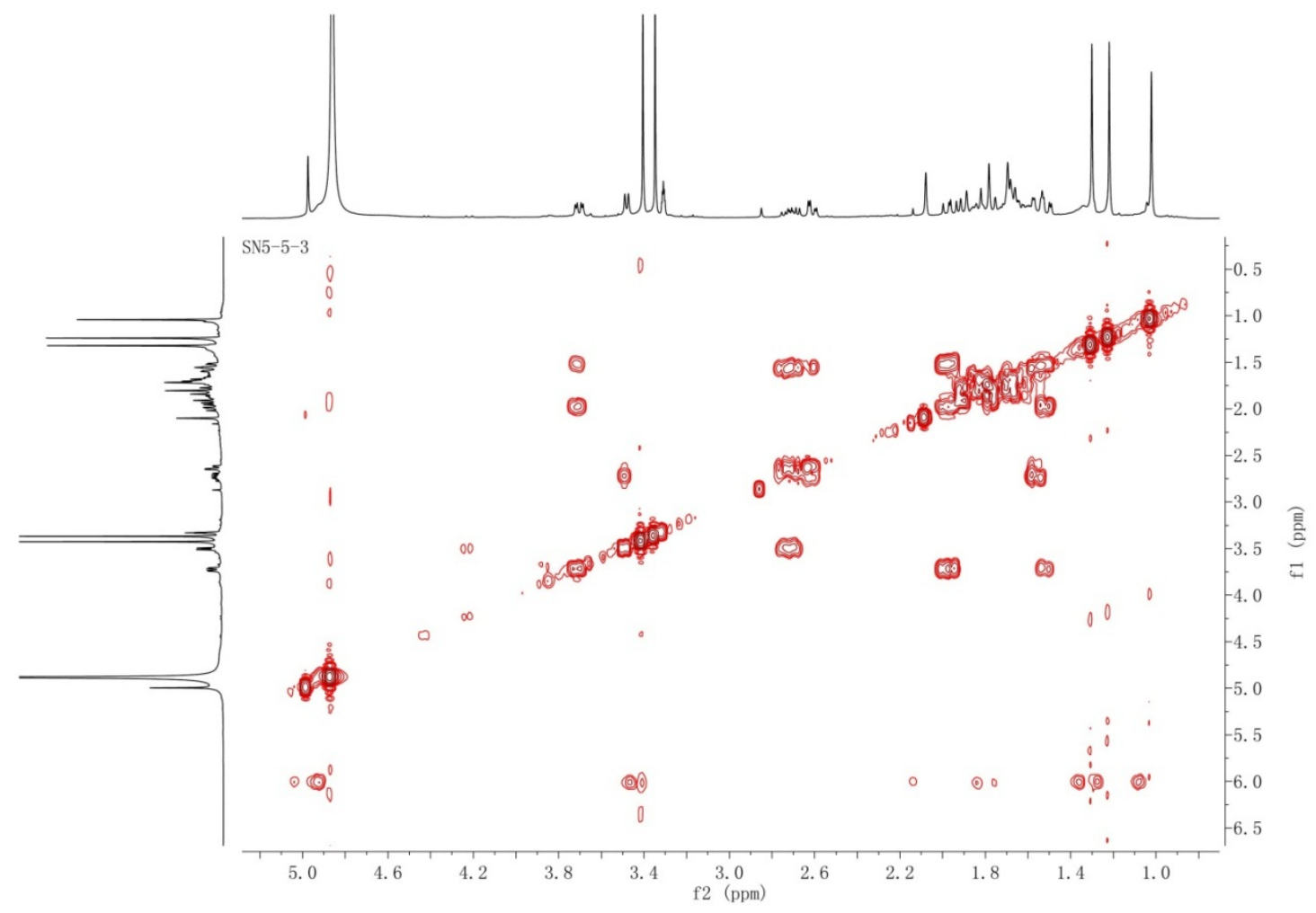

Figure S18. ${ }^{1} \mathrm{H}^{-1} \mathrm{H}$ COSY spectrum (400 MHz, methanol- $d_{4}$ ) of compound 1

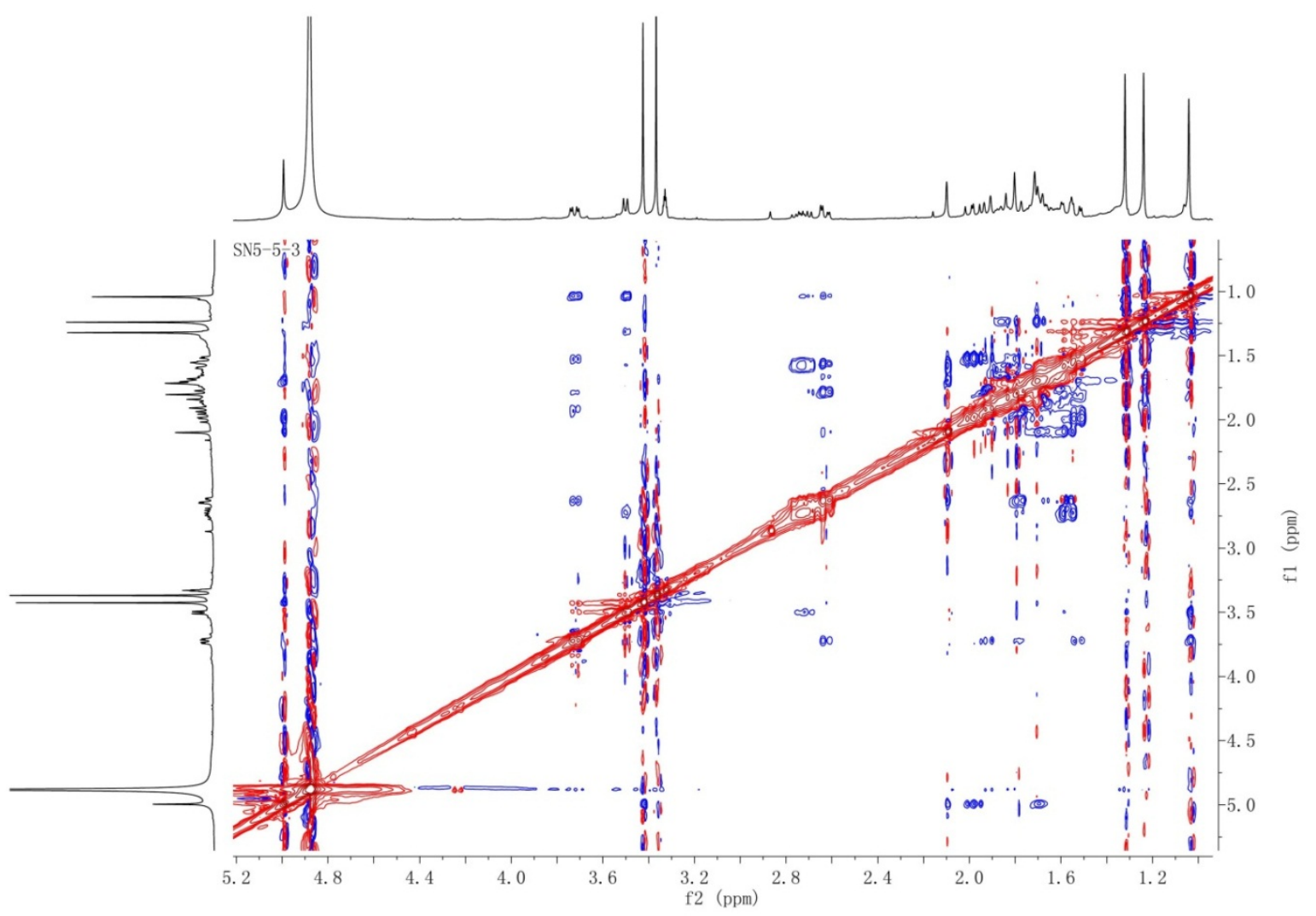

Figure S19. NOESY spectrum (400 MHz, methanol- $d_{4}$ ) of compound 1 


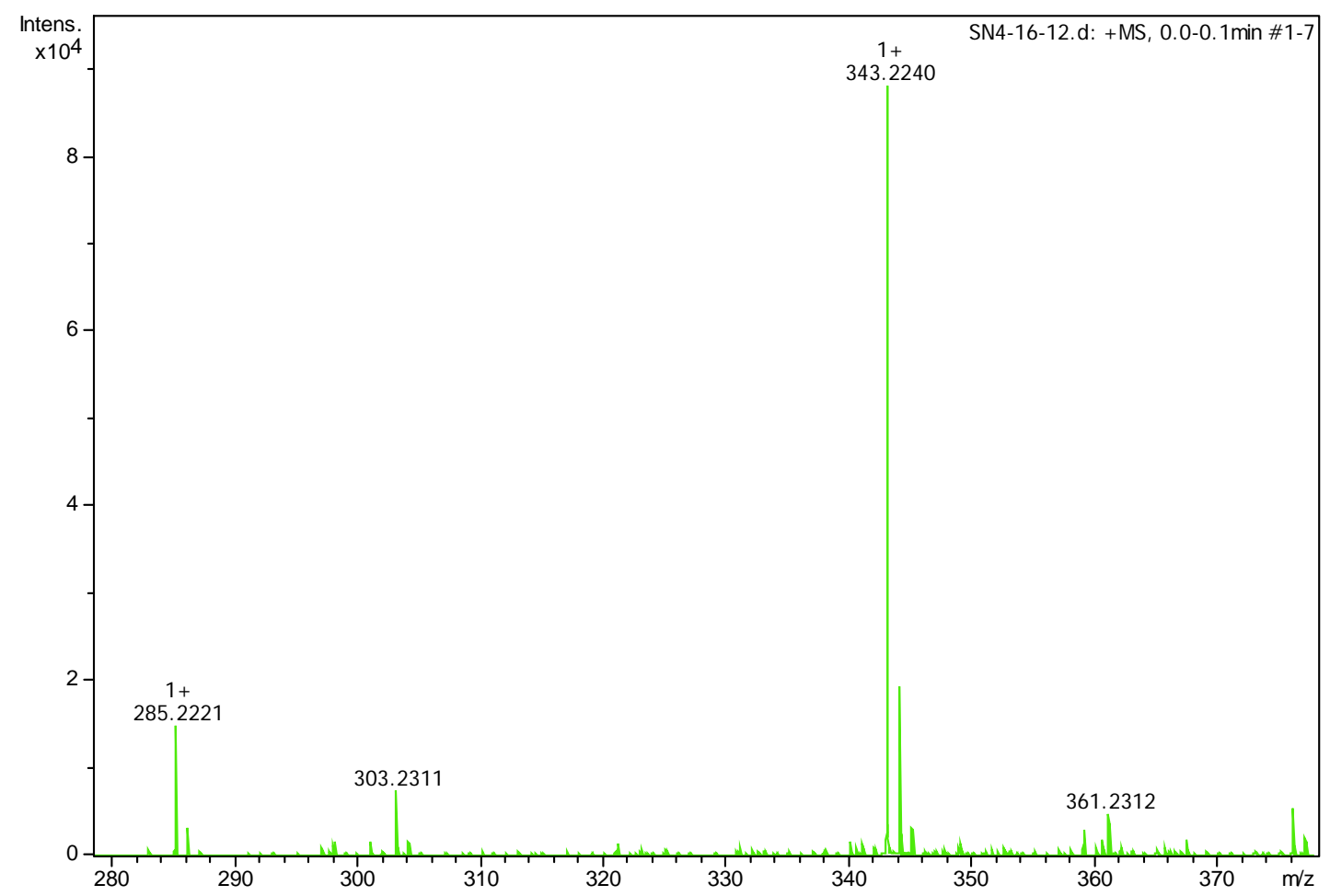

Figure S20. (+)-HRESIMS of compound 2

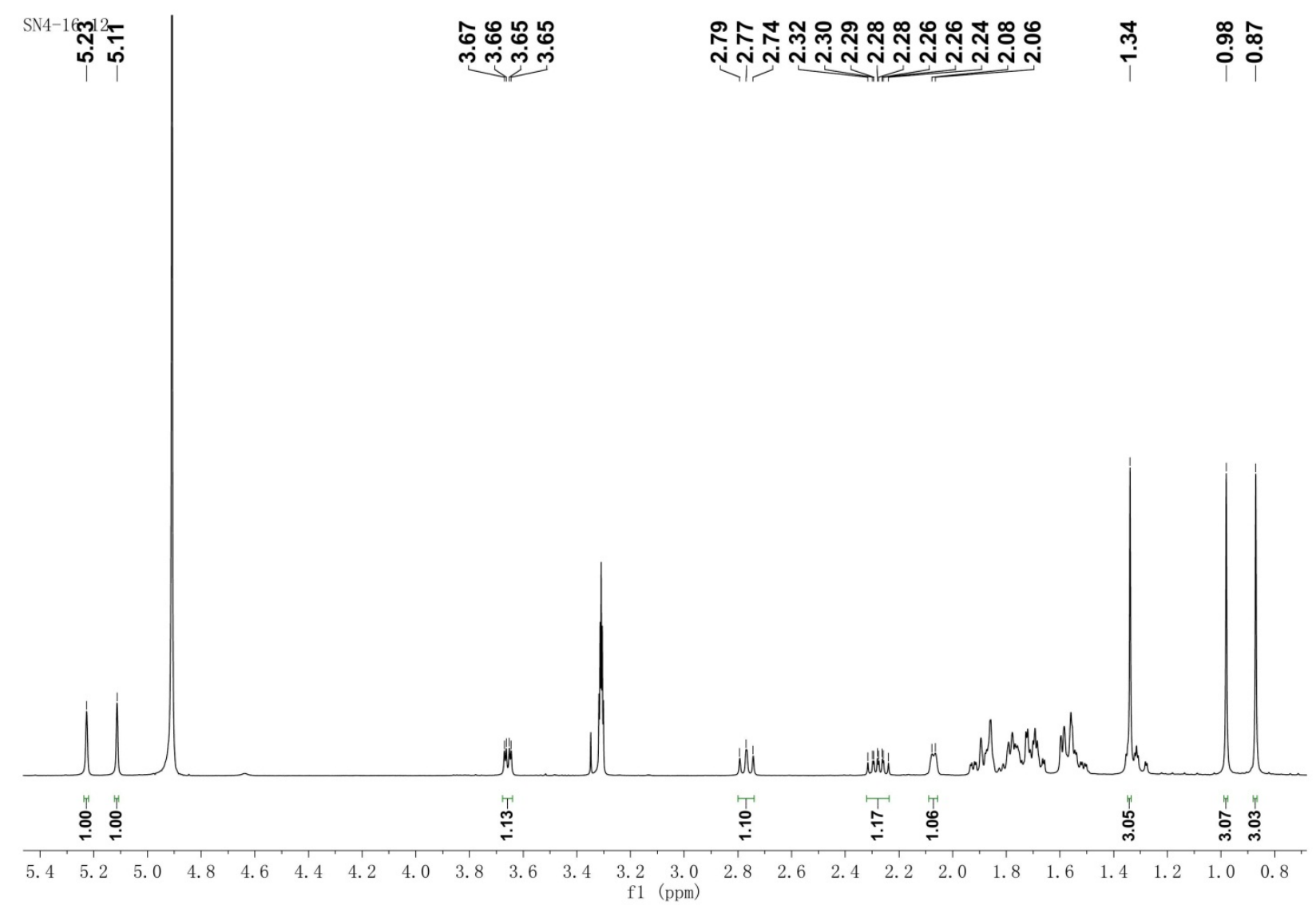

Figure S21. ${ }^{1} \mathrm{H}$ NMR (400 MHz, methanol- $\left.d_{4}\right)$ spectrum of compound 2 


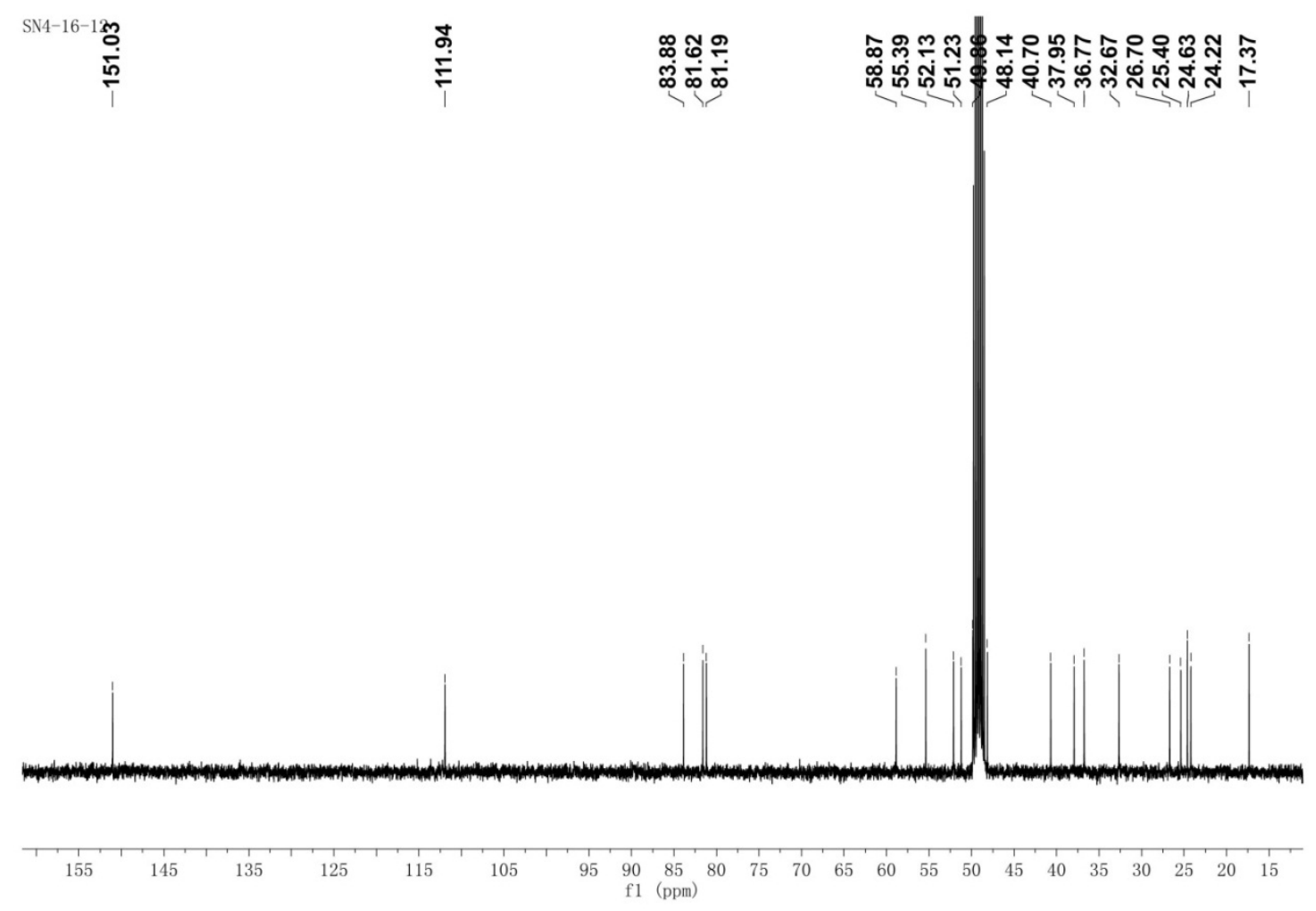

Figure S22. ${ }^{13} \mathrm{C}$ NMR $\left(100 \mathrm{MHz}\right.$, methanol- $\left.d_{4}\right)$ spectrum of compound 2

SN4-16-1๕0

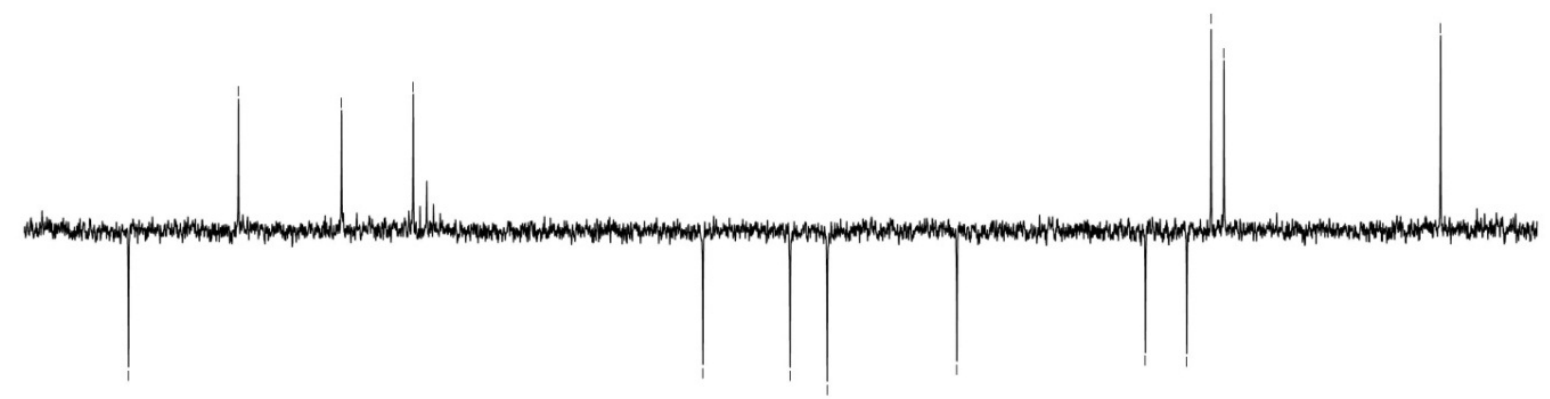

\begin{tabular}{llllllllllllllllllllllllllllll}
\hline 62 & 60 & 58 & 56 & 54 & 52 & 50 & 48 & 46 & 44 & 42 & 40 & 38 & 36 & 34 & 32 & 30 & 28 & 26 & 24 & 22 & 20 & 18 & 16
\end{tabular}

Figure S23. DEPT (100 MHz, methanol- $\left.d_{4}\right)$ spectrum of compound 2 


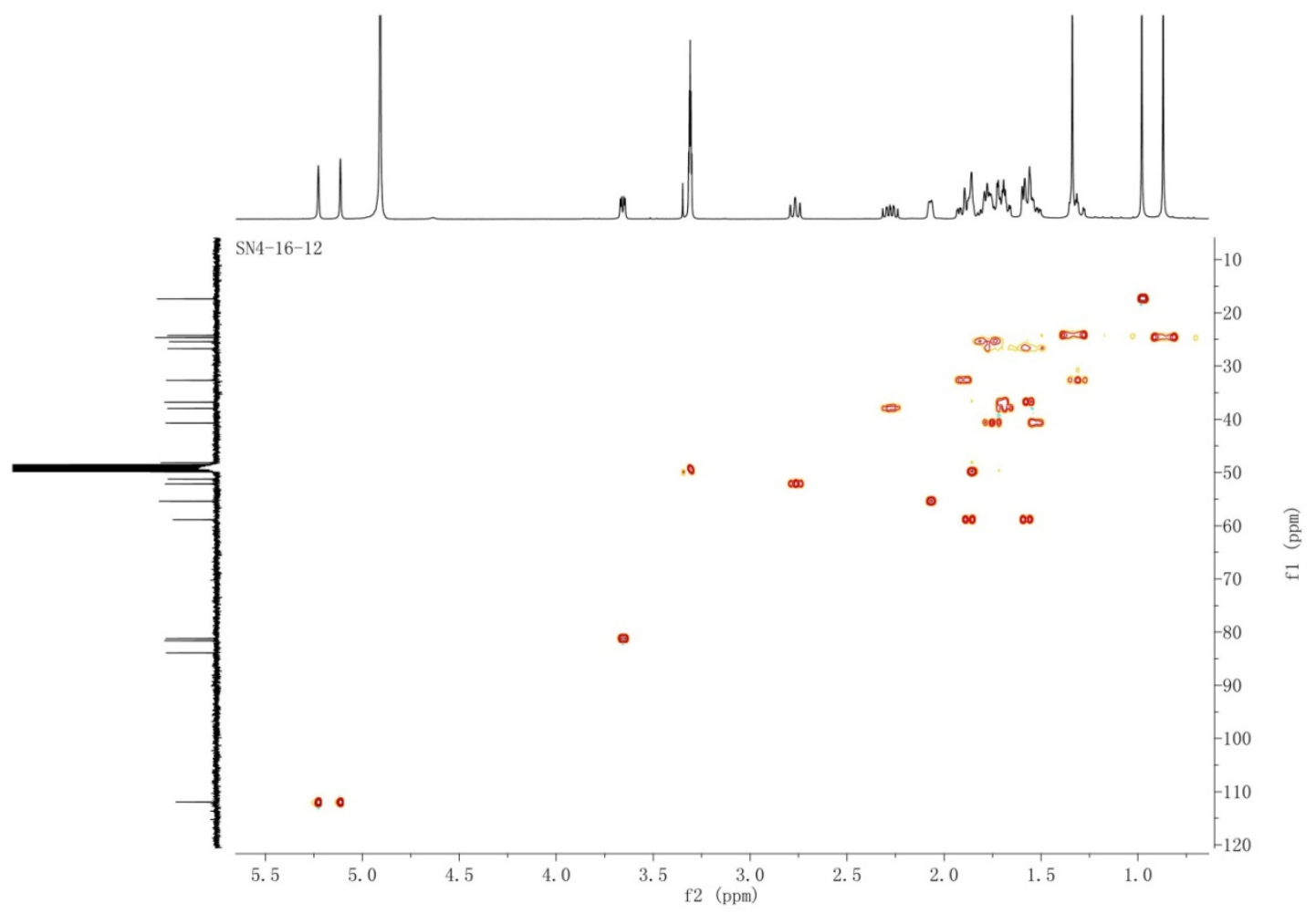

Figure S24. HSQC (methanol- $\left.d_{4}\right)$ spectrum of compound $2\left({ }^{1} \mathrm{H}: 400 \mathrm{MHz},{ }^{13} \mathrm{C}: 100 \mathrm{MHz}\right)$

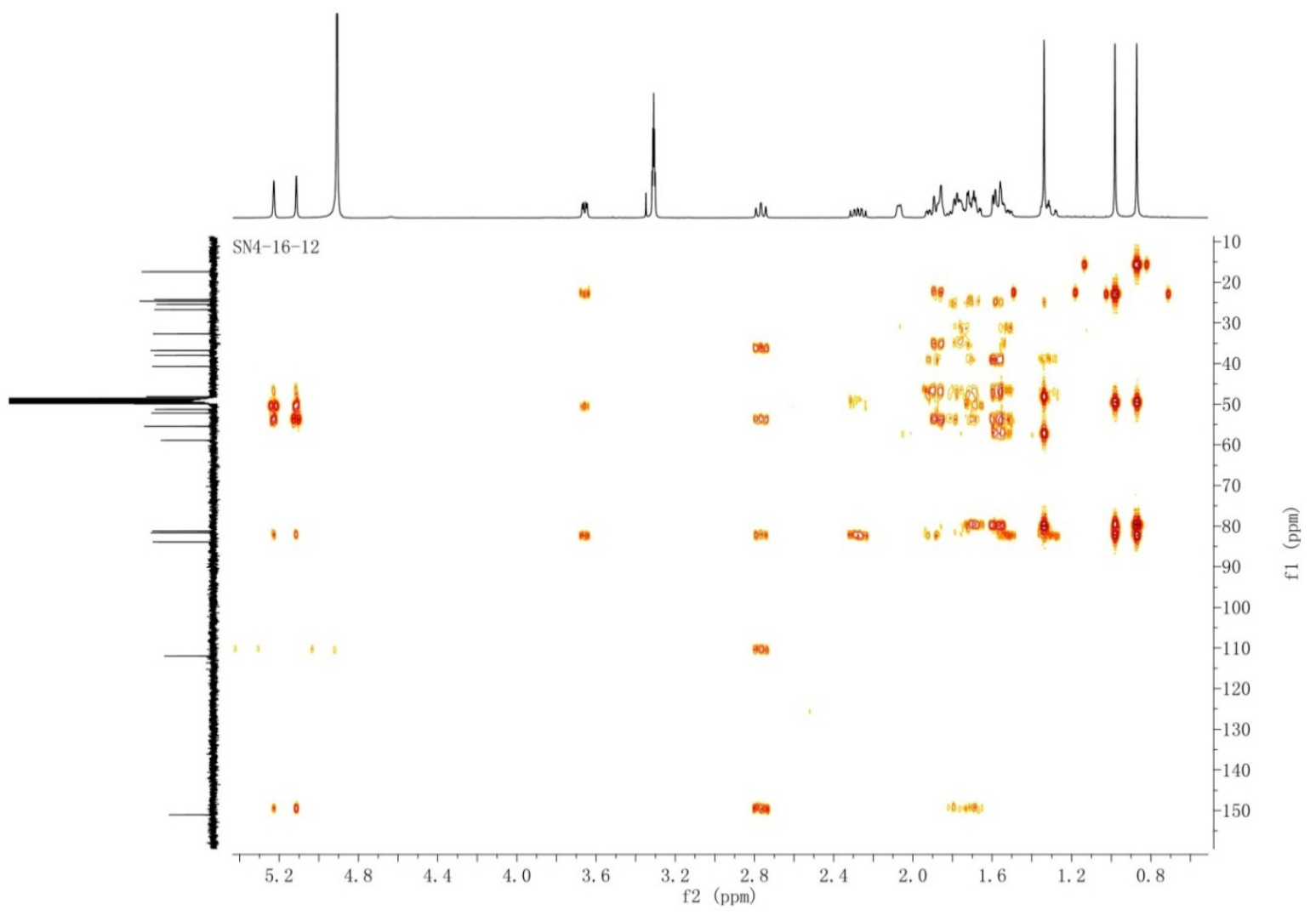

Figure S25. HMBC (methanol- $\left.d_{4}\right)$ spectrum of compound $2\left({ }^{1} \mathrm{H}: 400 \mathrm{MHz},{ }^{13} \mathrm{C}: 100 \mathrm{MHz}\right)$ 


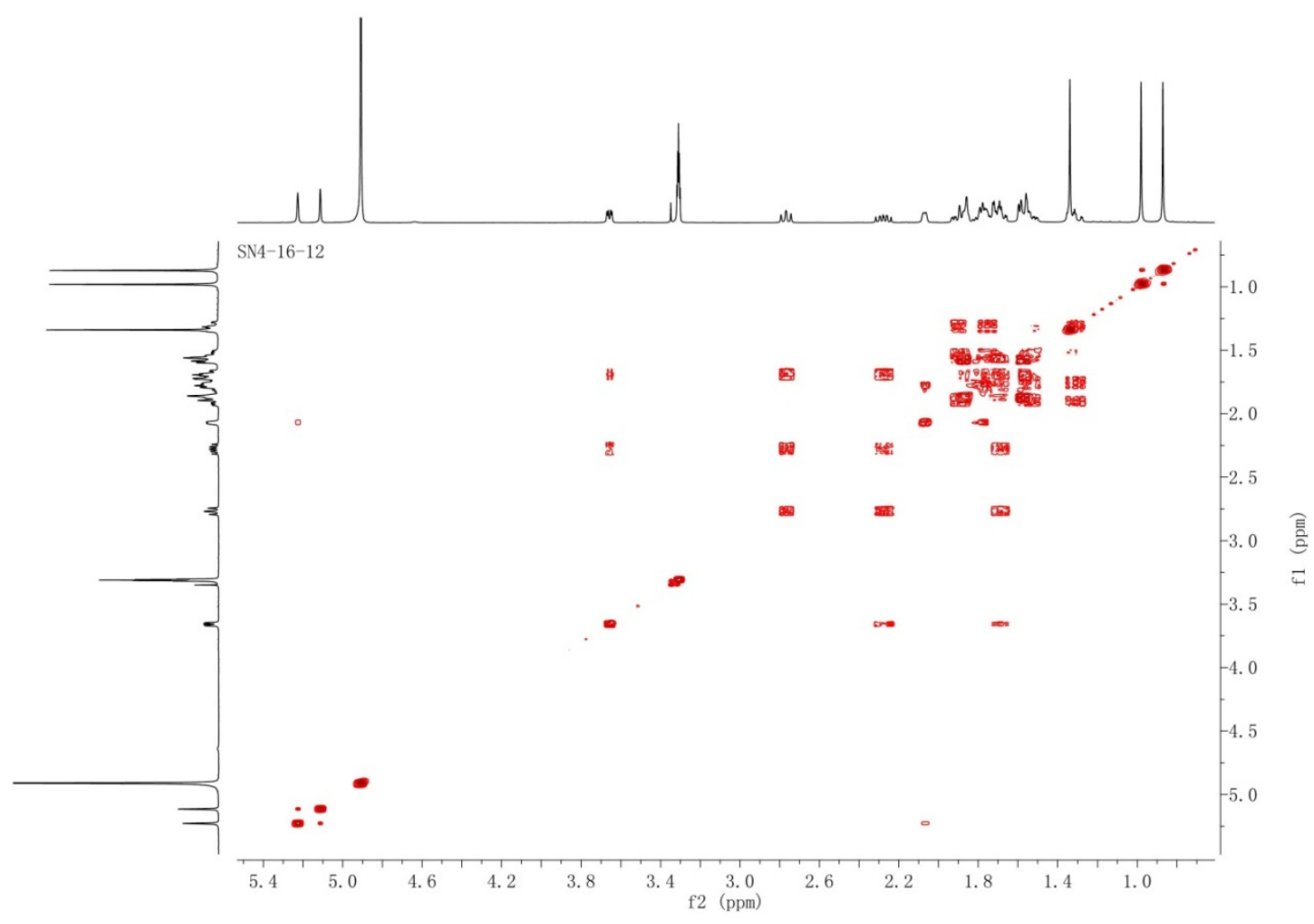

Figure S26. ${ }^{1} \mathrm{H}-{ }^{1} \mathrm{H}$ COSY (400 MHz, methanol- $\left.d_{4}\right)$ spectrum of compound 2

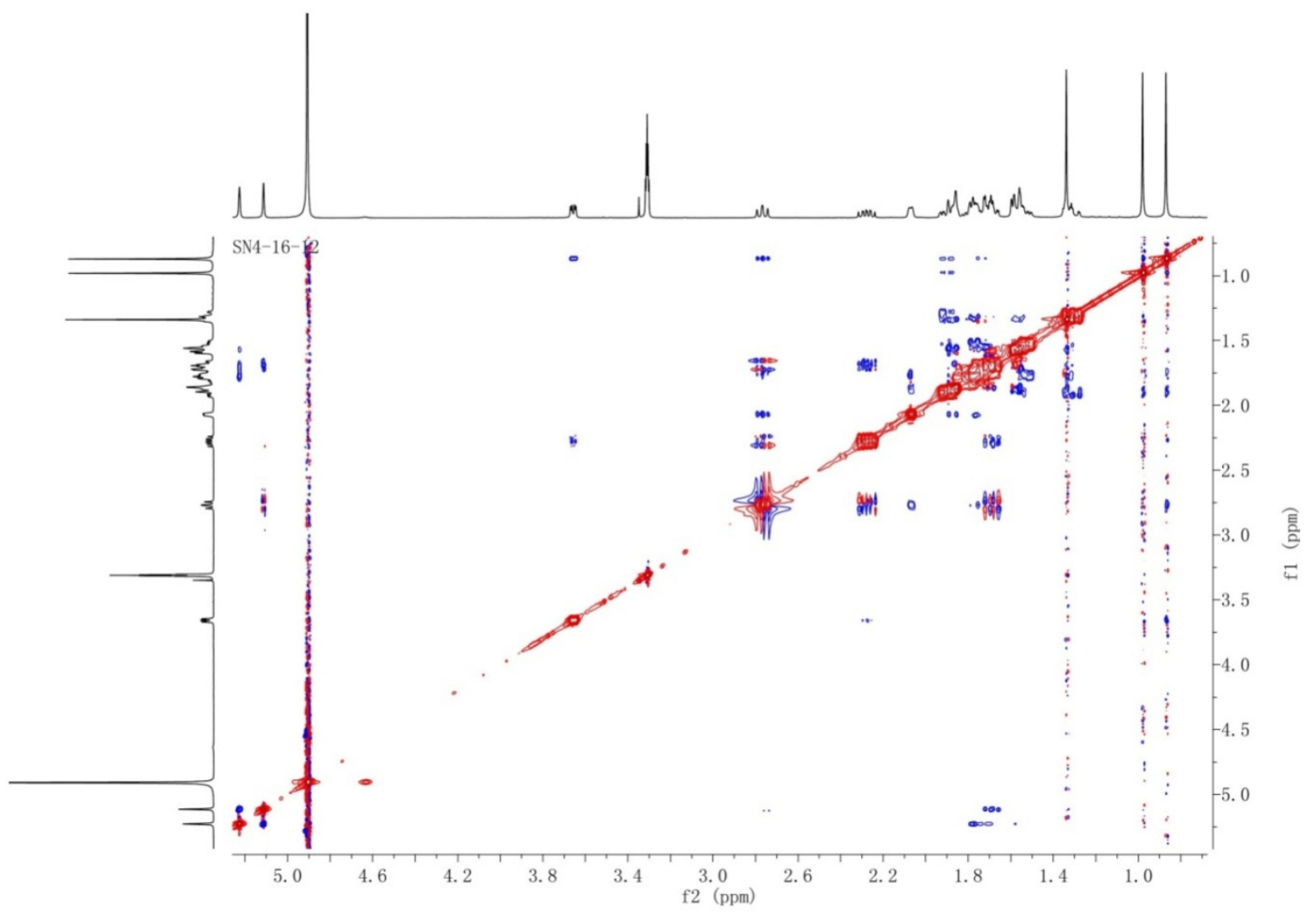

Figure S27. NOESY spectrum (400 MHz, methanol- $d_{4}$ ) of compound 2 


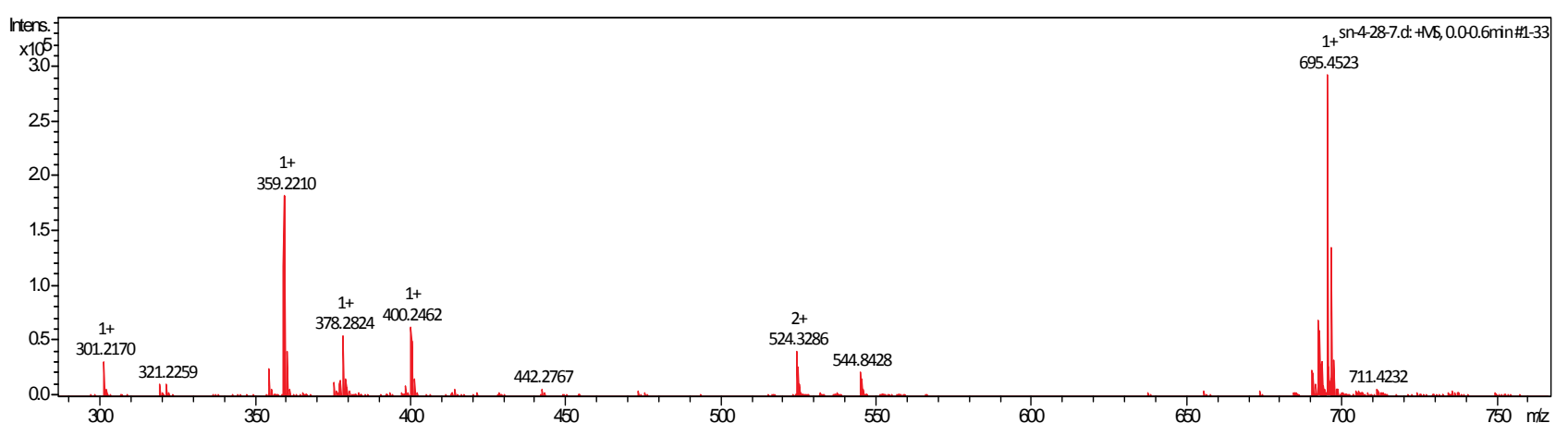

Figure S28. (+)-HRESIMS of compound 3

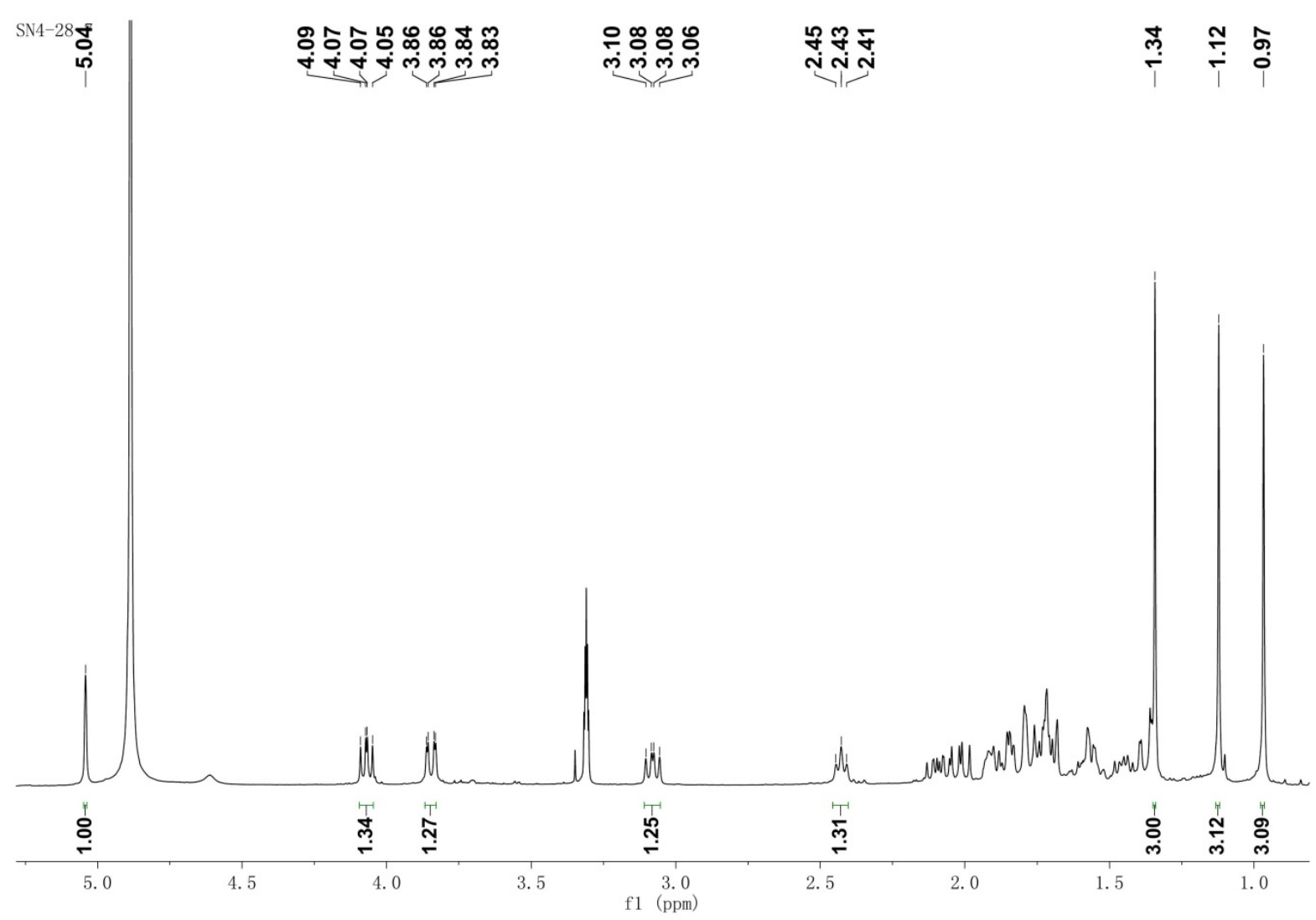

Figure S29. ${ }^{1} \mathrm{H}$ NMR (400 MHz, methanol- $d_{4}$ ) spectrum of compound 3 


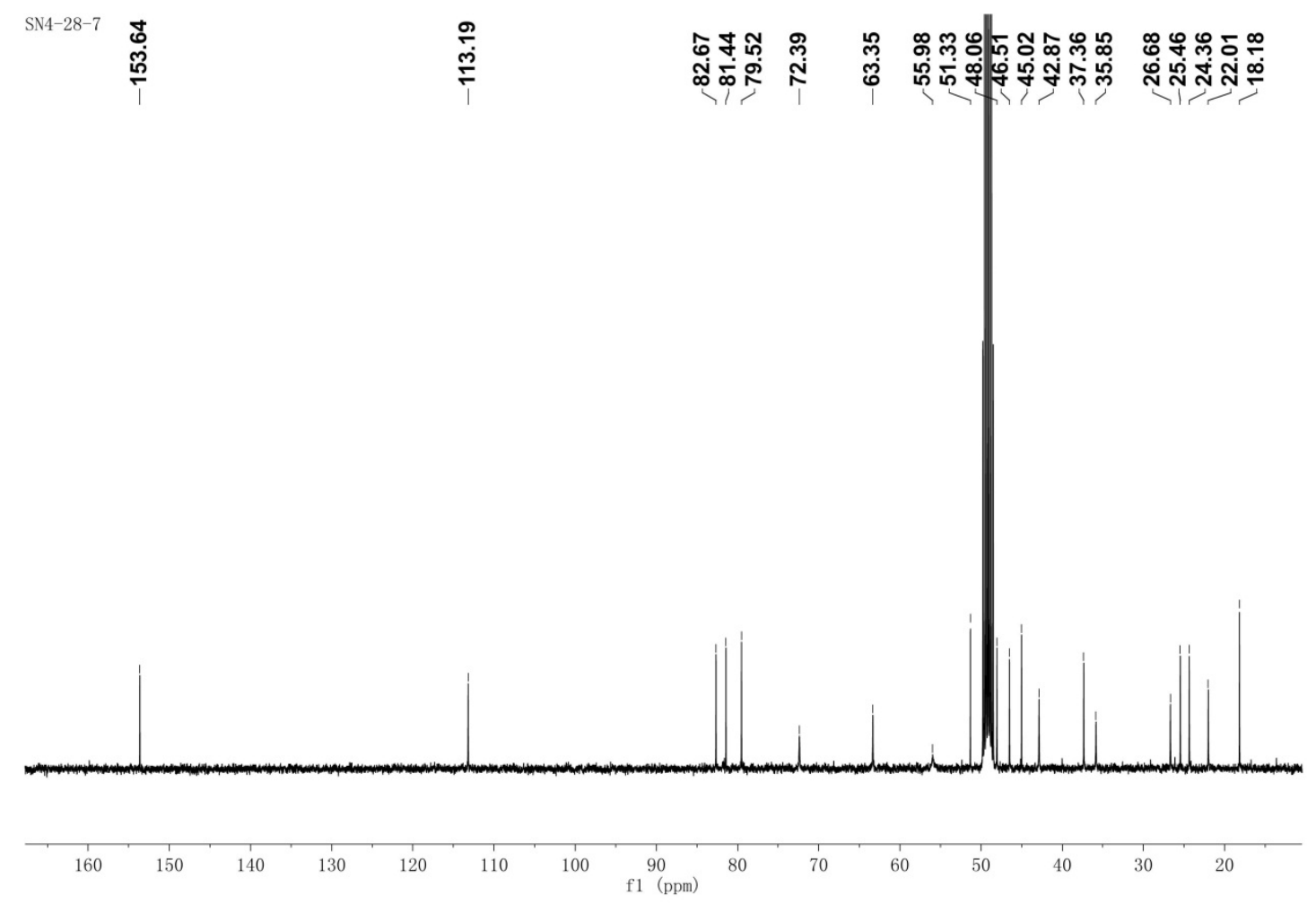

Figure S30. ${ }^{13} \mathrm{C}$ NMR $\left(100 \mathrm{MHz}\right.$, methanol- $\left.d_{4}\right)$ spectrum of compound 3

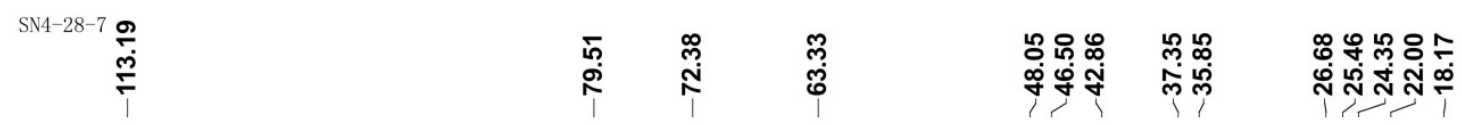

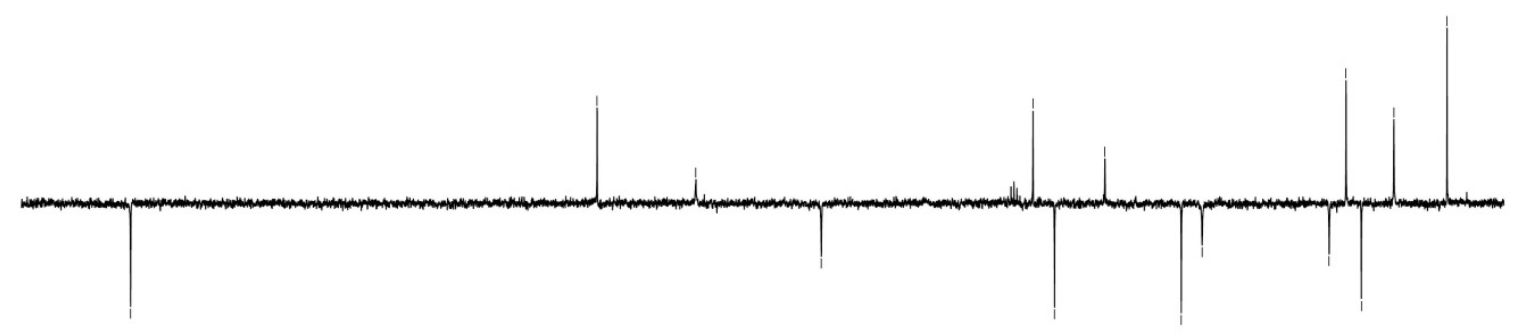

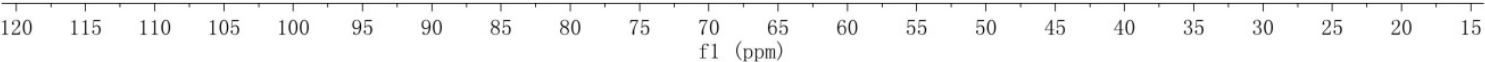

Figure S31. DEPT (100 MHz, methanol- $\left.d_{4}\right)$ spectrum of compound 3 


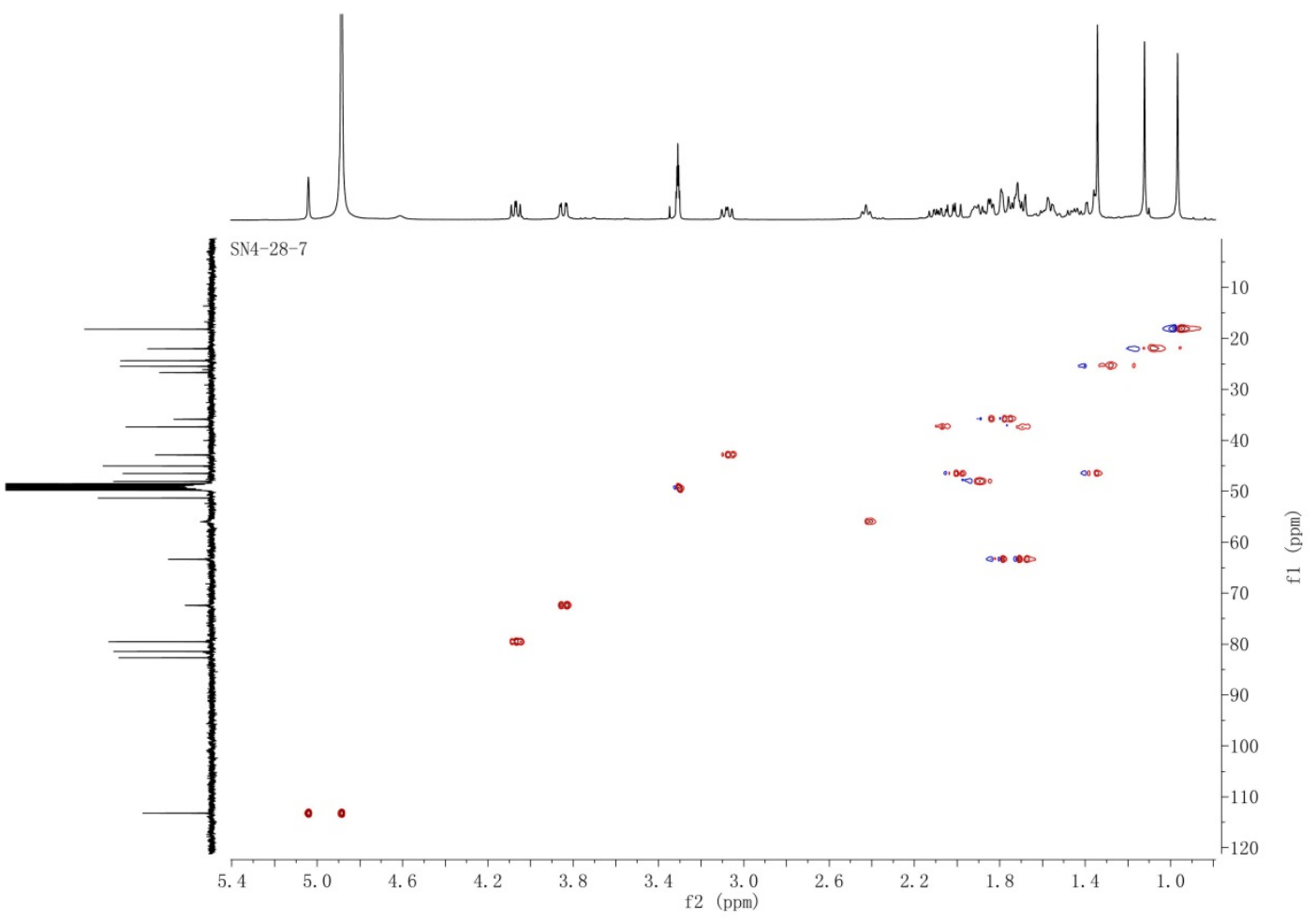

Figure S32. HSQC (methanol- $\left.d_{4}\right)$ spectrum of compound $3\left({ }^{1} \mathrm{H}: 400 \mathrm{MHz},{ }^{13} \mathrm{C}: 100 \mathrm{MHz}\right)$

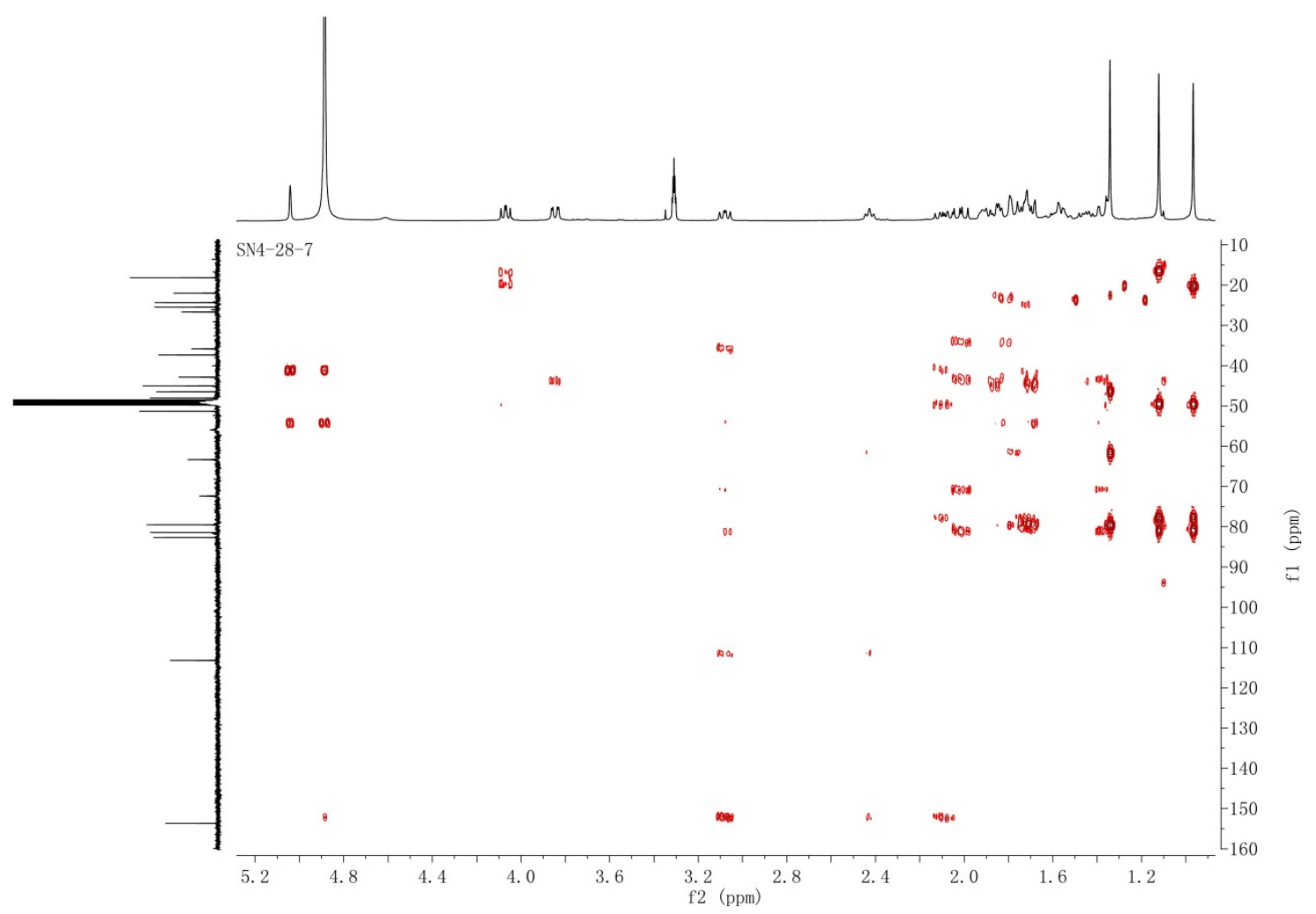

Figure S33. HMBC (methanol- $\left.d_{4}\right)$ spectrum of compound $3\left({ }^{1} \mathrm{H}: 400 \mathrm{MHz},{ }^{13} \mathrm{C}: 100 \mathrm{MHz}\right)$ 


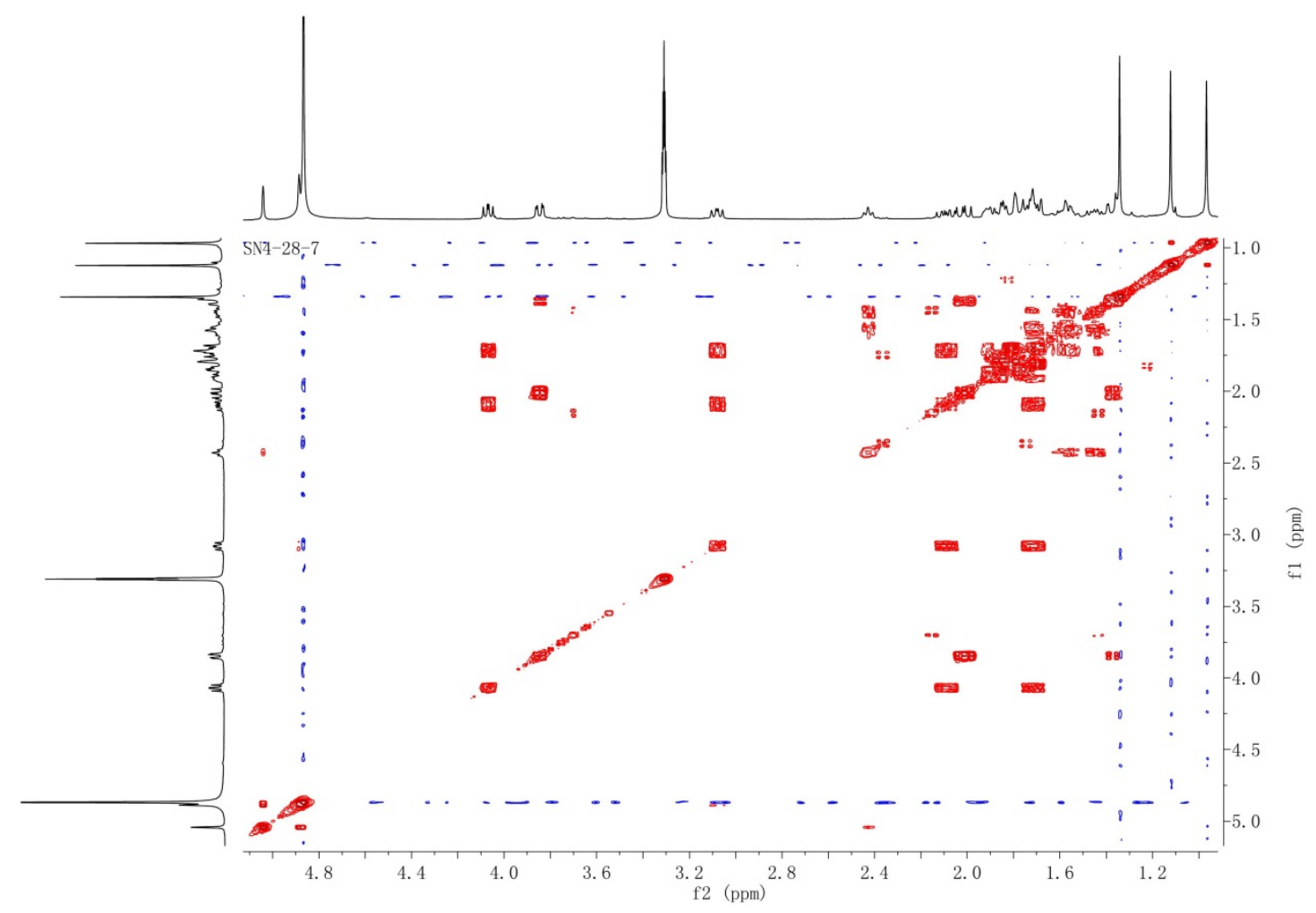

Figure $\mathrm{S} 34 .{ }^{1} \mathrm{H}-{ }^{1} \mathrm{H}$ COSY $\left(400 \mathrm{MHz}\right.$, methanol- $\left.d_{4}\right)$ spectrum of compound 3

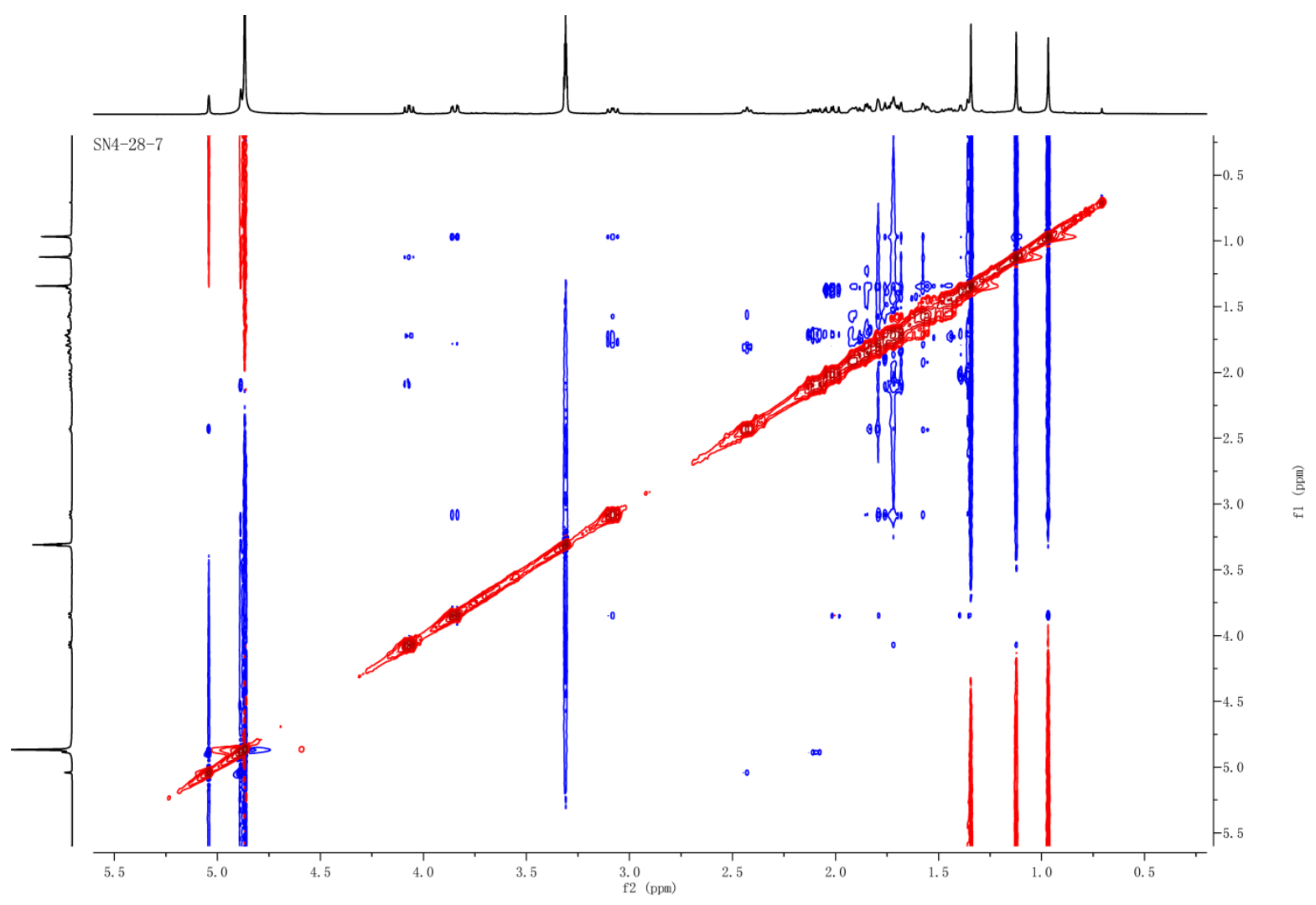

Figure S35. NOESY (400 MHz, methanol- $d_{4}$ ) spectrum of compound 3 


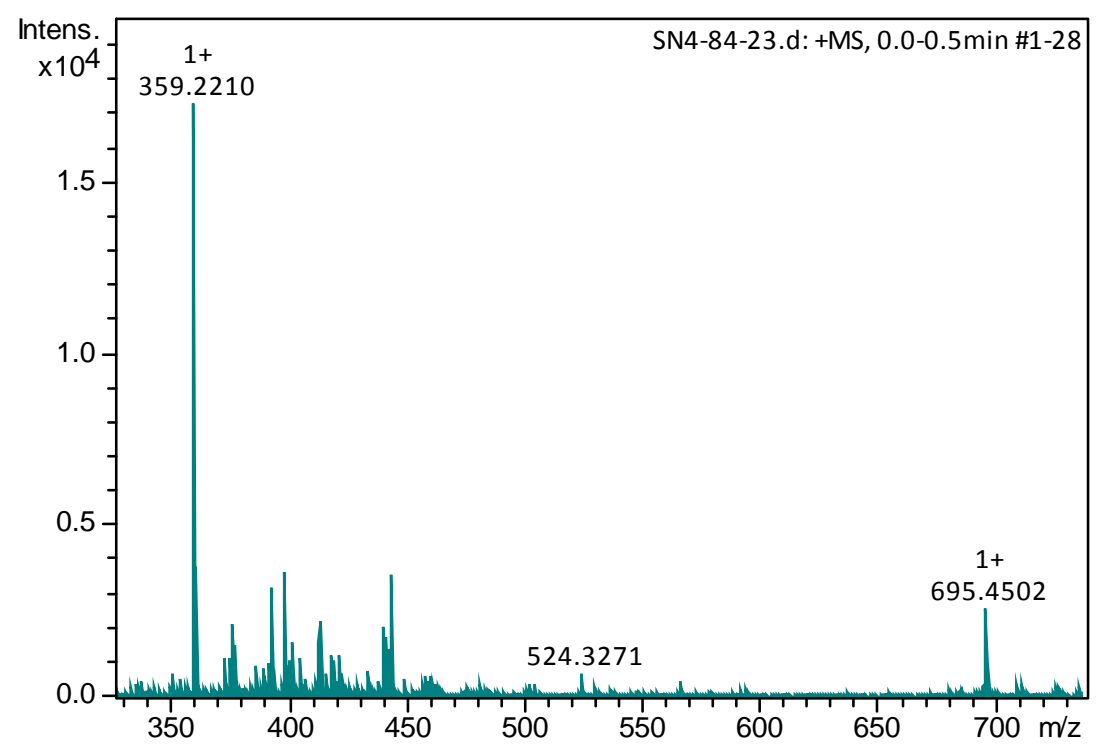

Figure S36. (+)-HRESIMS of compound 4

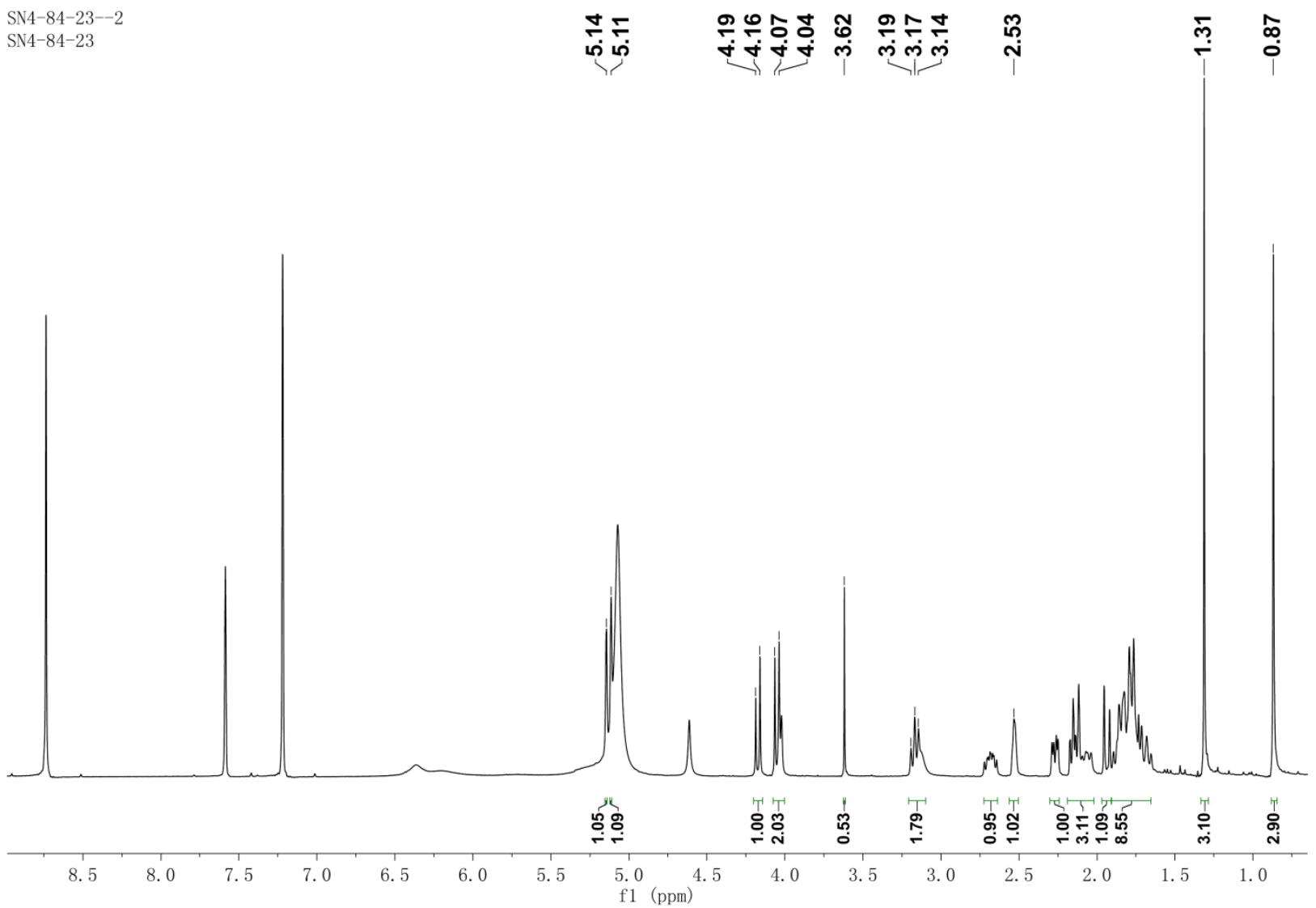

Figure S37. ${ }^{1} \mathrm{H}$ NMR (400 MHz, pyridine- $d_{5}$ ) spectrum of compound 4 

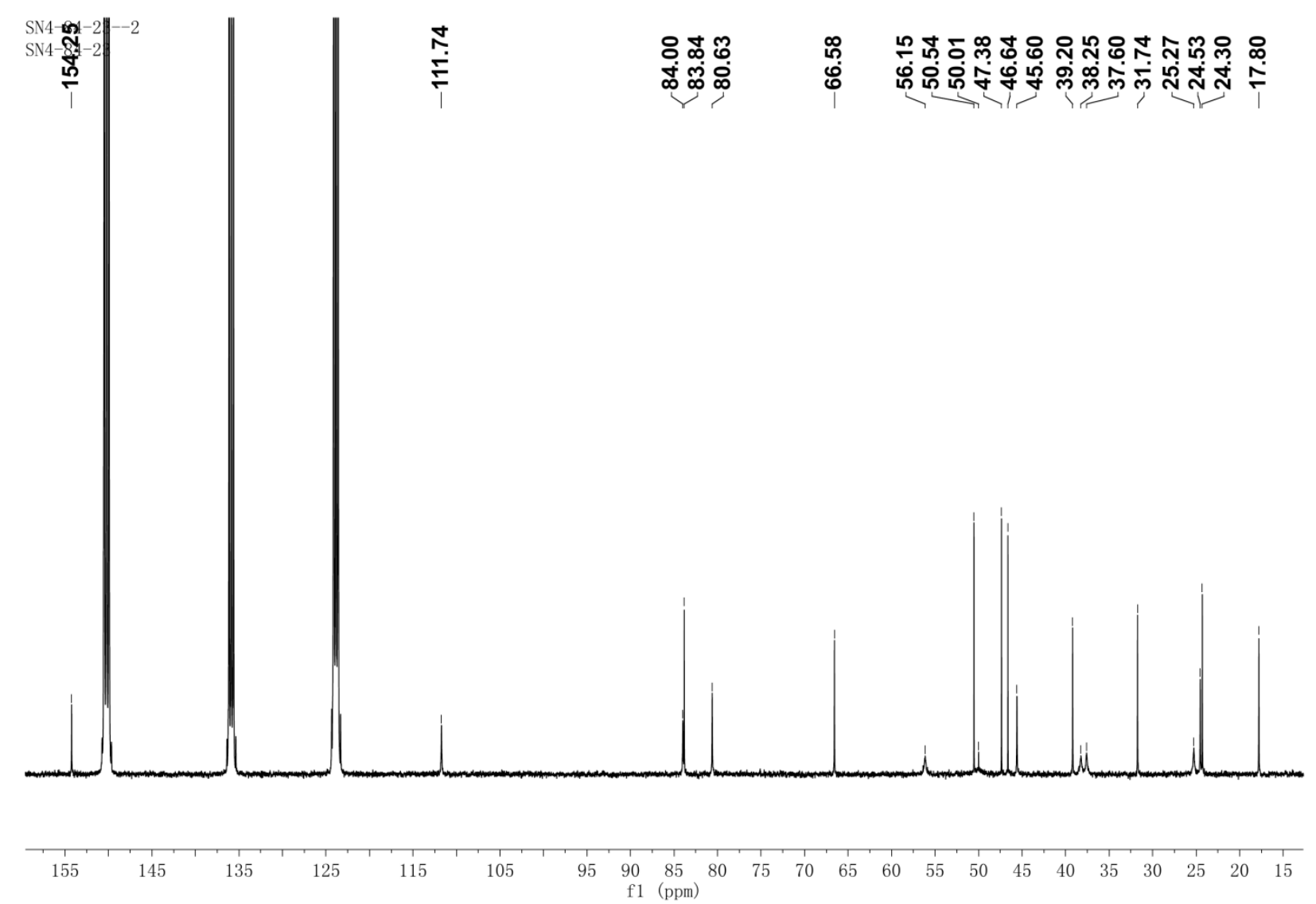

Figure S38. ${ }^{13} \mathrm{C}$ NMR (100 MHz, pyridine- $\left.d_{5}\right)$ spectrum of compound 4

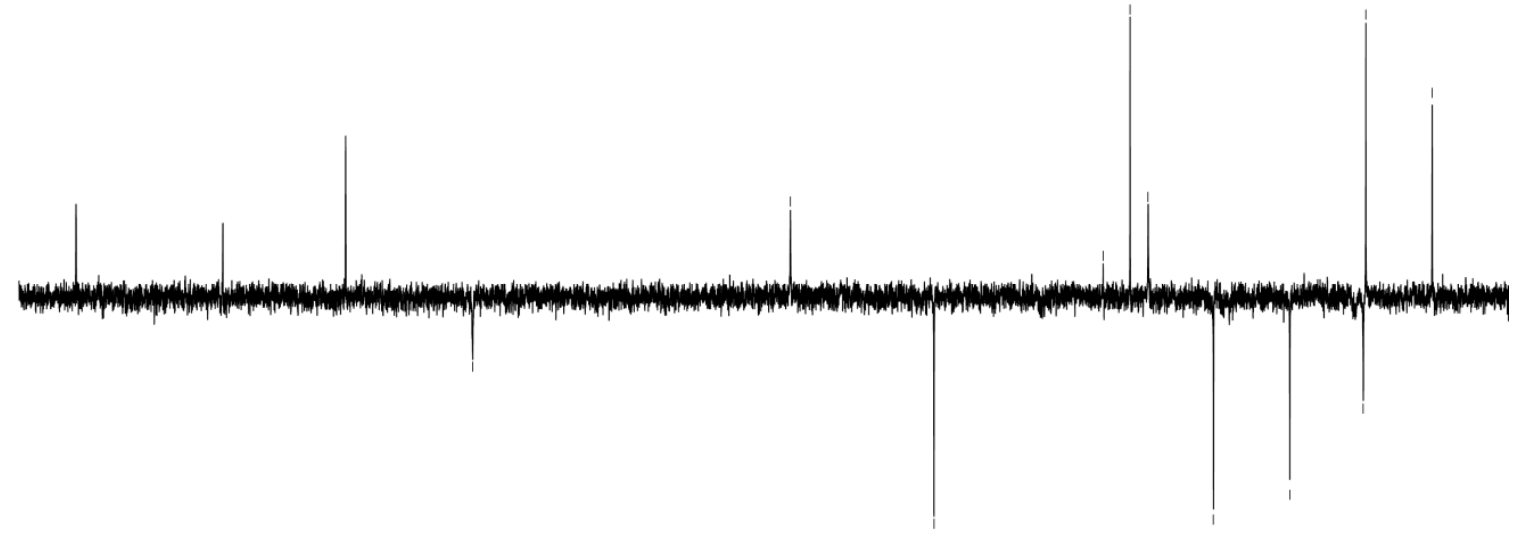

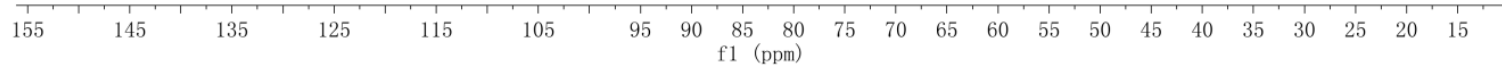

Figure S39. DEPT (100 MHz, pyridine- $\left.d_{5}\right)$ spectrum of compound 4 


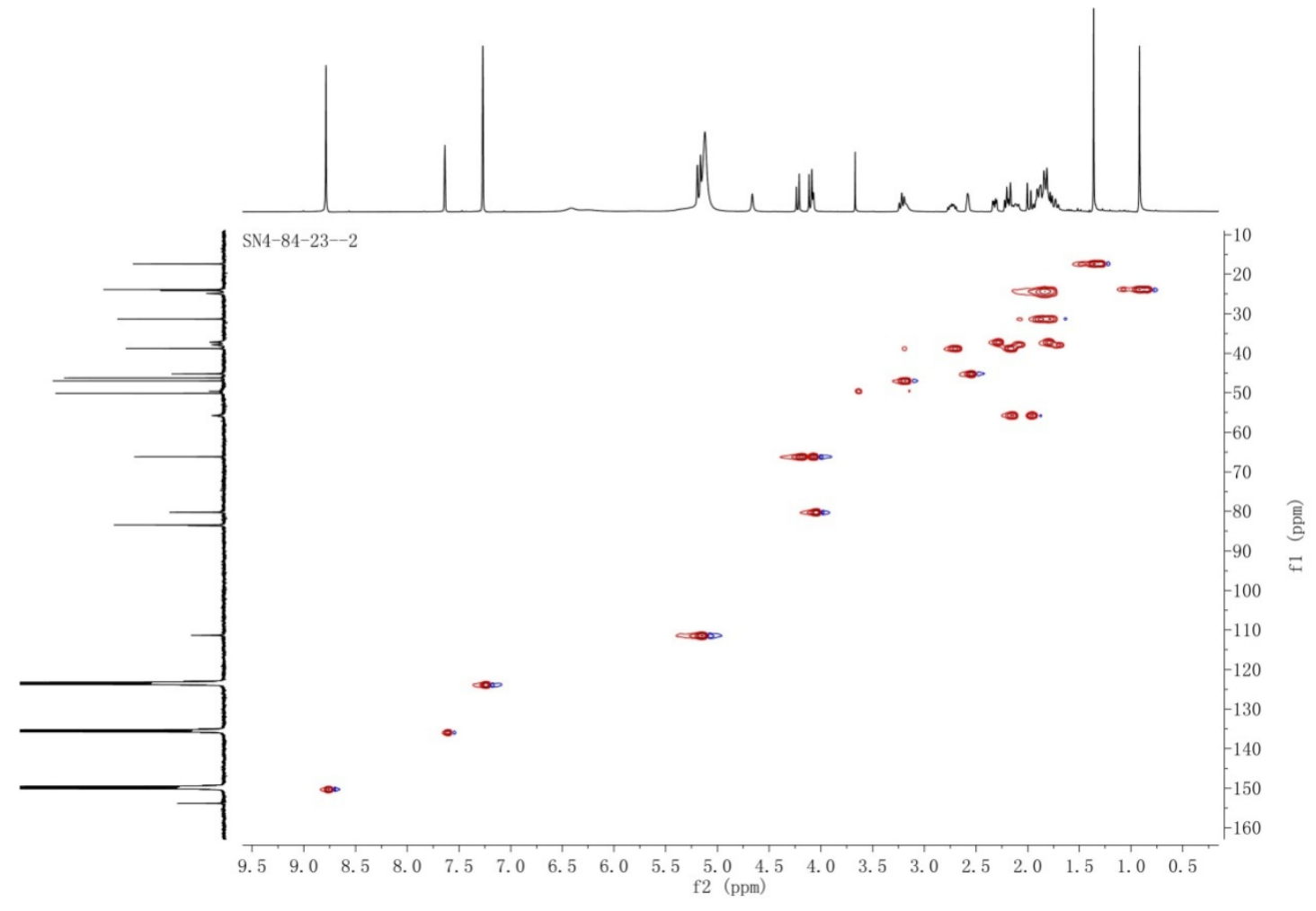

Figure S40. HSQC (pyridine- $d_{5}$ ) spectrum of compound $4\left({ }^{1} \mathrm{H}: 400 \mathrm{MHz},{ }^{13} \mathrm{C}: 100 \mathrm{MHz}\right)$

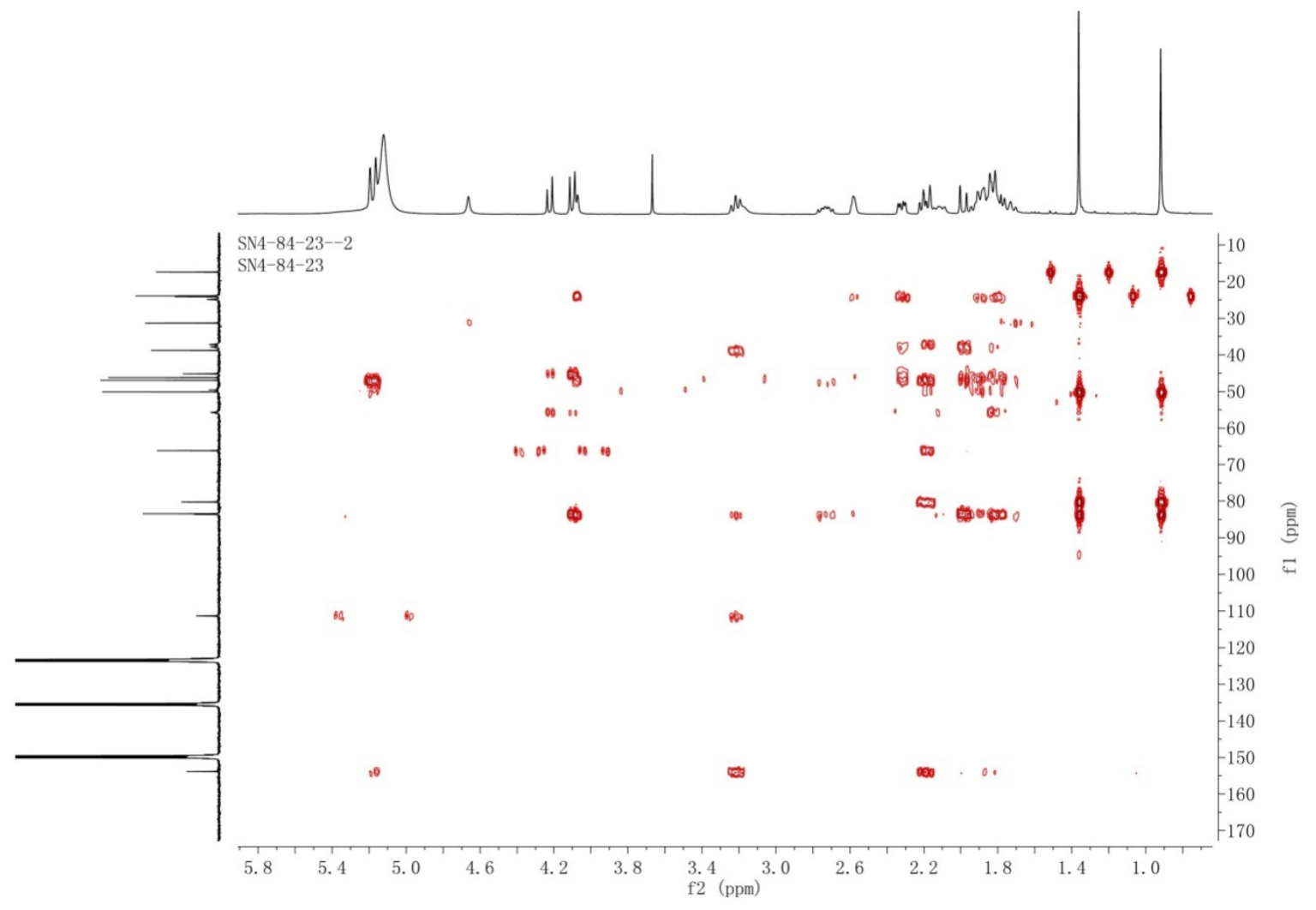

Figure S41. HMBC (pyridine- $d_{5}$ ) spectrum of compound $4\left({ }^{1} \mathrm{H}: 400 \mathrm{MHz},{ }^{13} \mathrm{C}: 100 \mathrm{MHz}\right)$ 


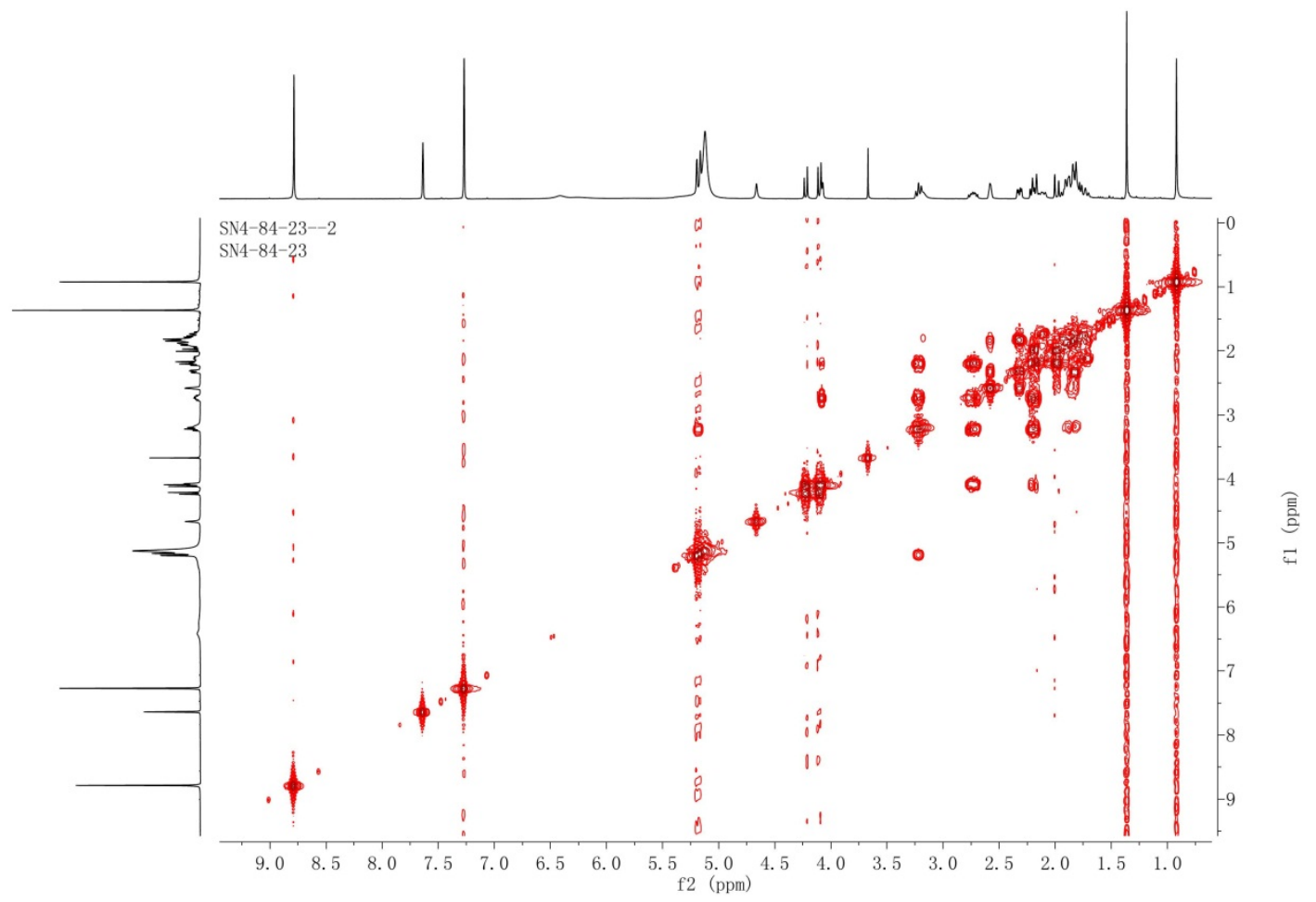

Figure $\mathrm{S} 42$. The ${ }^{1} \mathrm{H}^{-1} \mathrm{H}$ COSY (400 MHz, pyridine- $d_{5}$ ) spectrum of compound 4

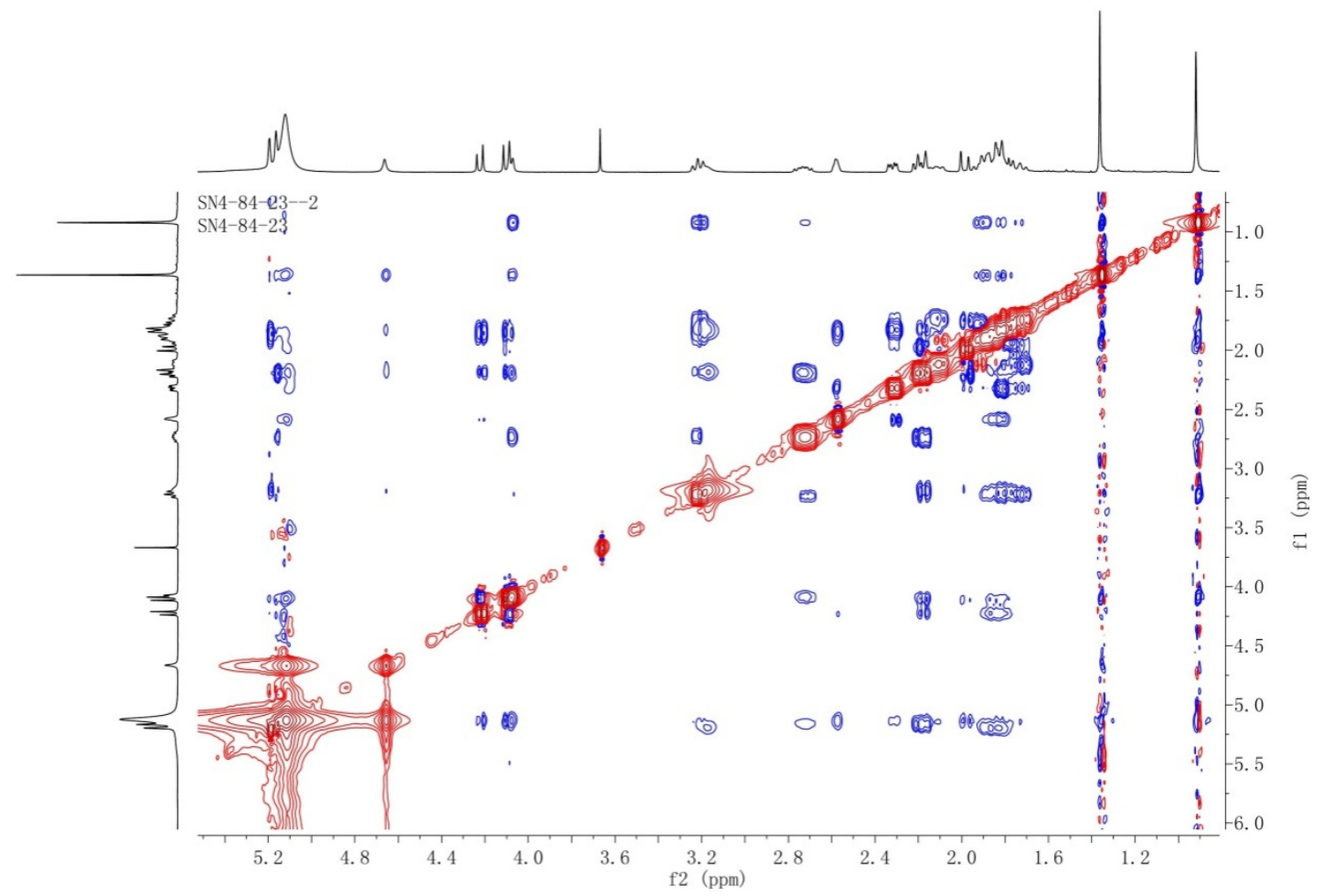

Figure S43. The NOESY ( $400 \mathrm{MHz}$, pyridine- $d_{5}$ ) spectrum of compound 4 


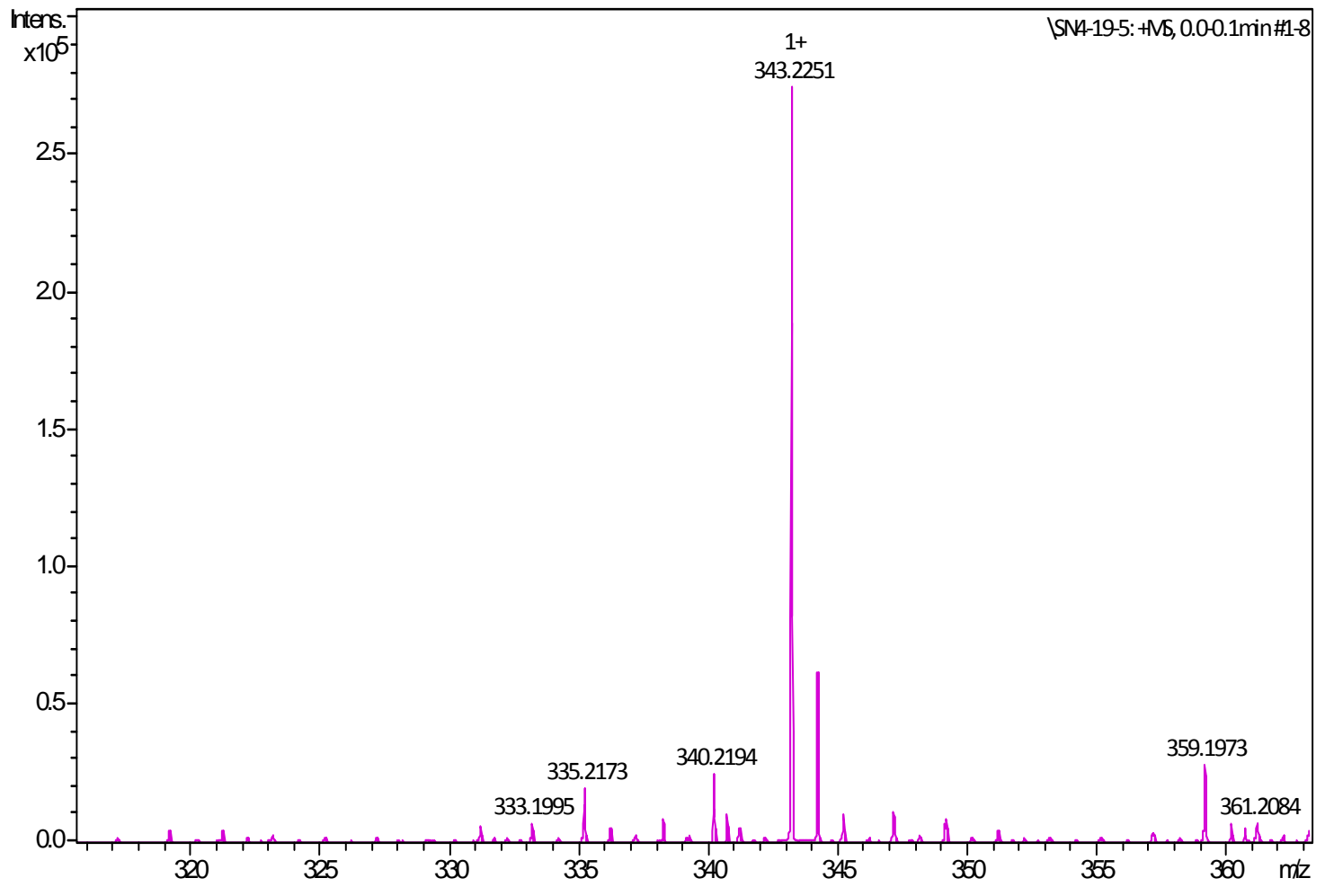

Figure S44. (+)-HRESIMS of compound 5

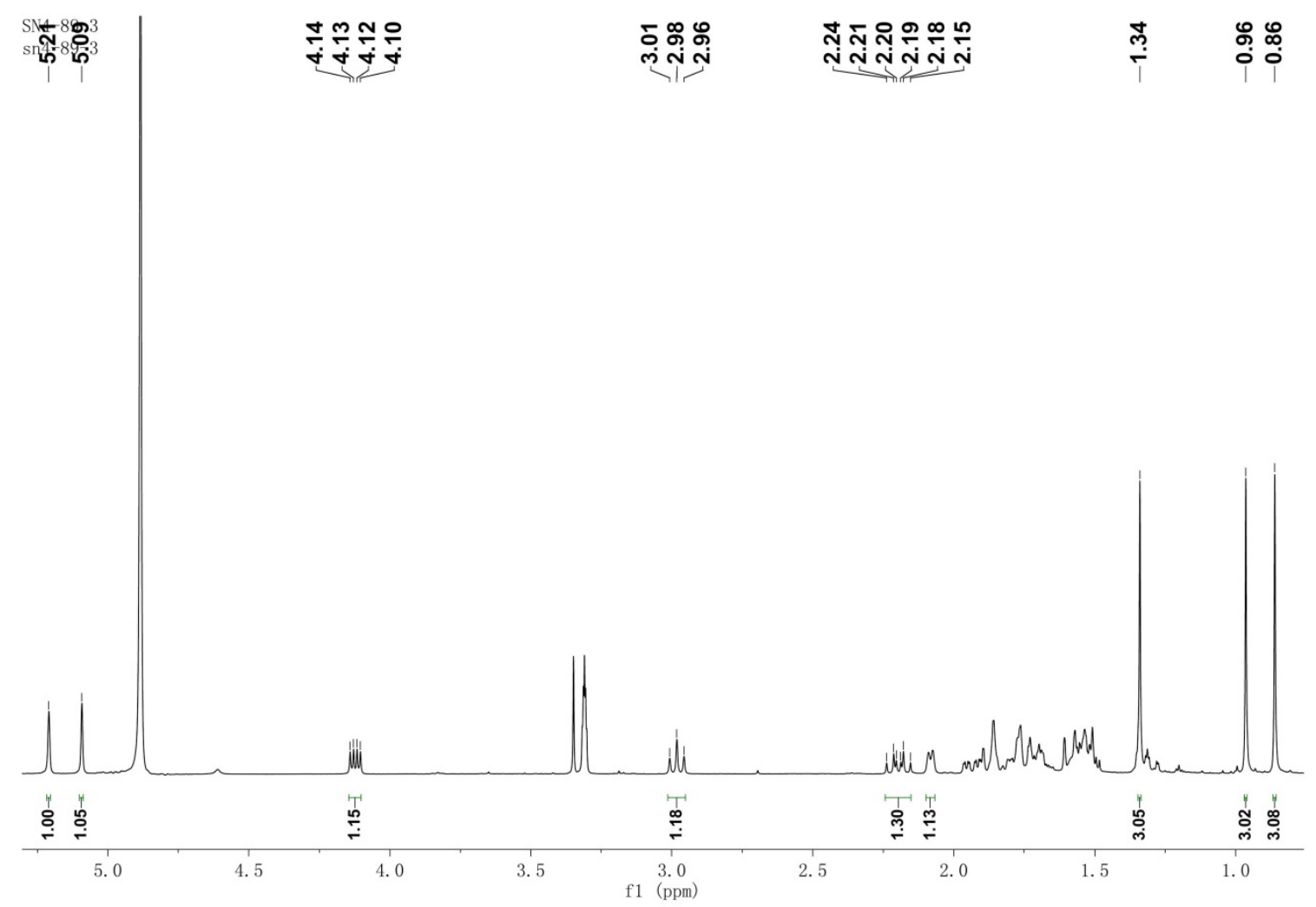

Figure S45. ${ }^{1} \mathrm{H}$ NMR (400 MHz, methanol- $d_{4}$ ) spectrum of compound 5 

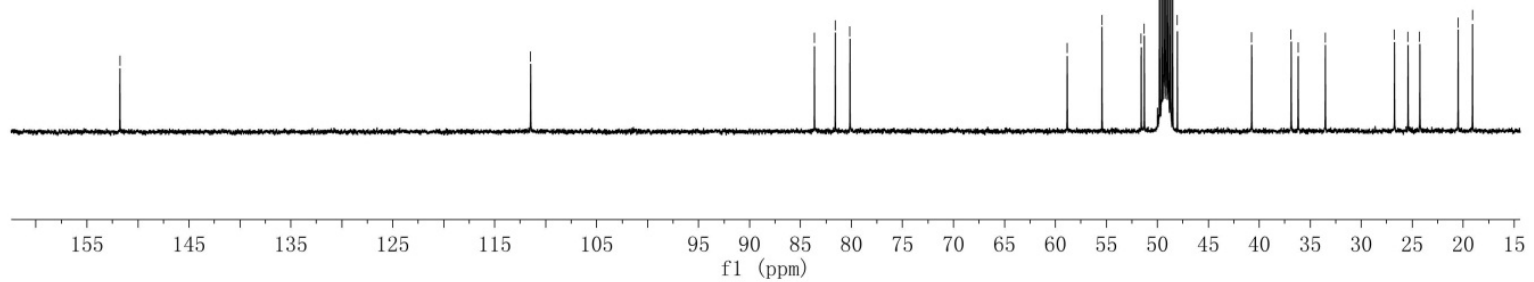

Figure S46. ${ }^{13} \mathrm{C}$ NMR $\left(100 \mathrm{MHz}\right.$, methanol- $\left.d_{4}\right)$ spectrum of compound 5

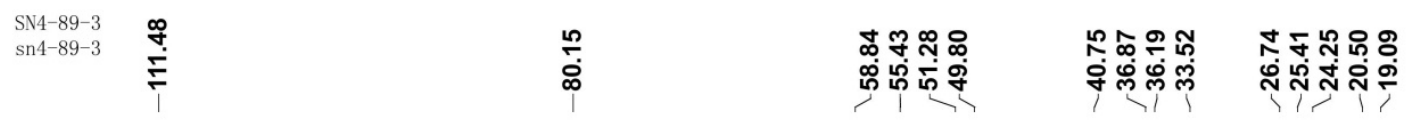

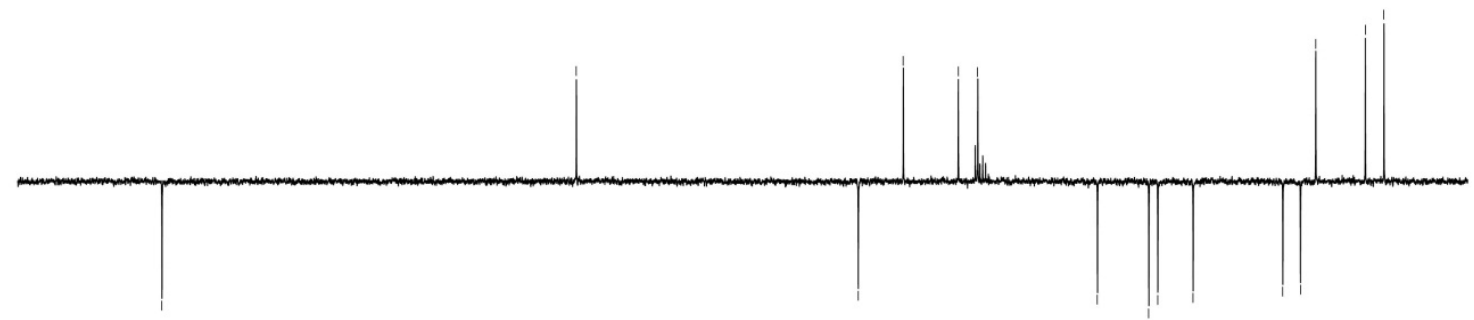

$\begin{array}{lllllllllllllllllllll}120 & 115 & 110 & 105 & 100 & 95 & 90 & 85 & 80 & 75 & \begin{array}{l}70 \\ \mathrm{f} 1\end{array}\left(\begin{array}{cc}65 \\ (\mathrm{pm})\end{array}\right. & 60 & 55 & 50 & 45 & 40 & 35 & 30 & 25 & 20 & 15\end{array}$

Figure S47. DEPT (100 MHz, methanol- $\left.d_{4}\right)$ spectrum of compound 5 


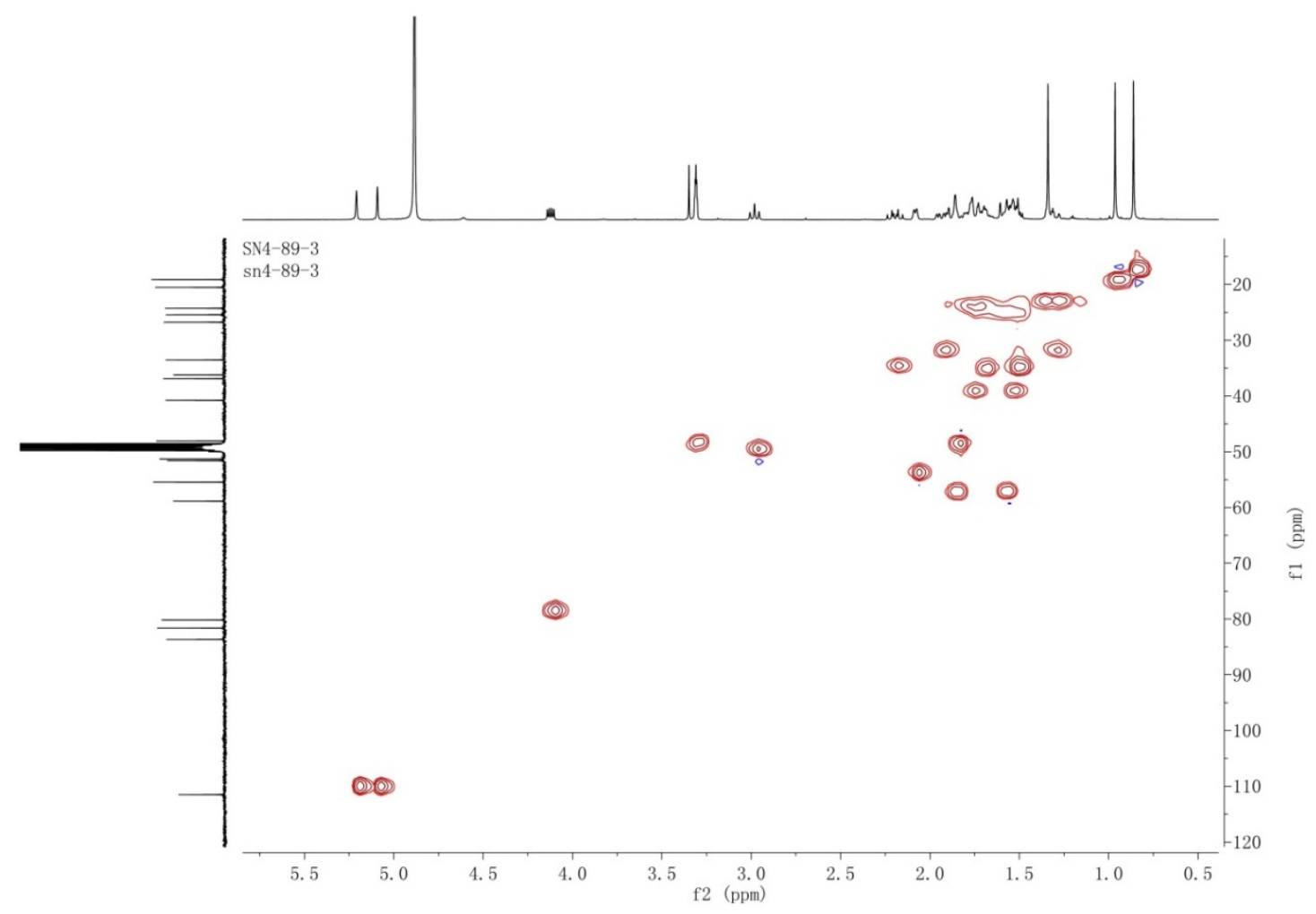

Figure S48. HSQC (methanol- $\left.d_{4}\right)$ spectrum of compound $5\left({ }^{1} \mathrm{H}: 400 \mathrm{MHz},{ }^{13} \mathrm{C}: 100 \mathrm{MHz}\right)$

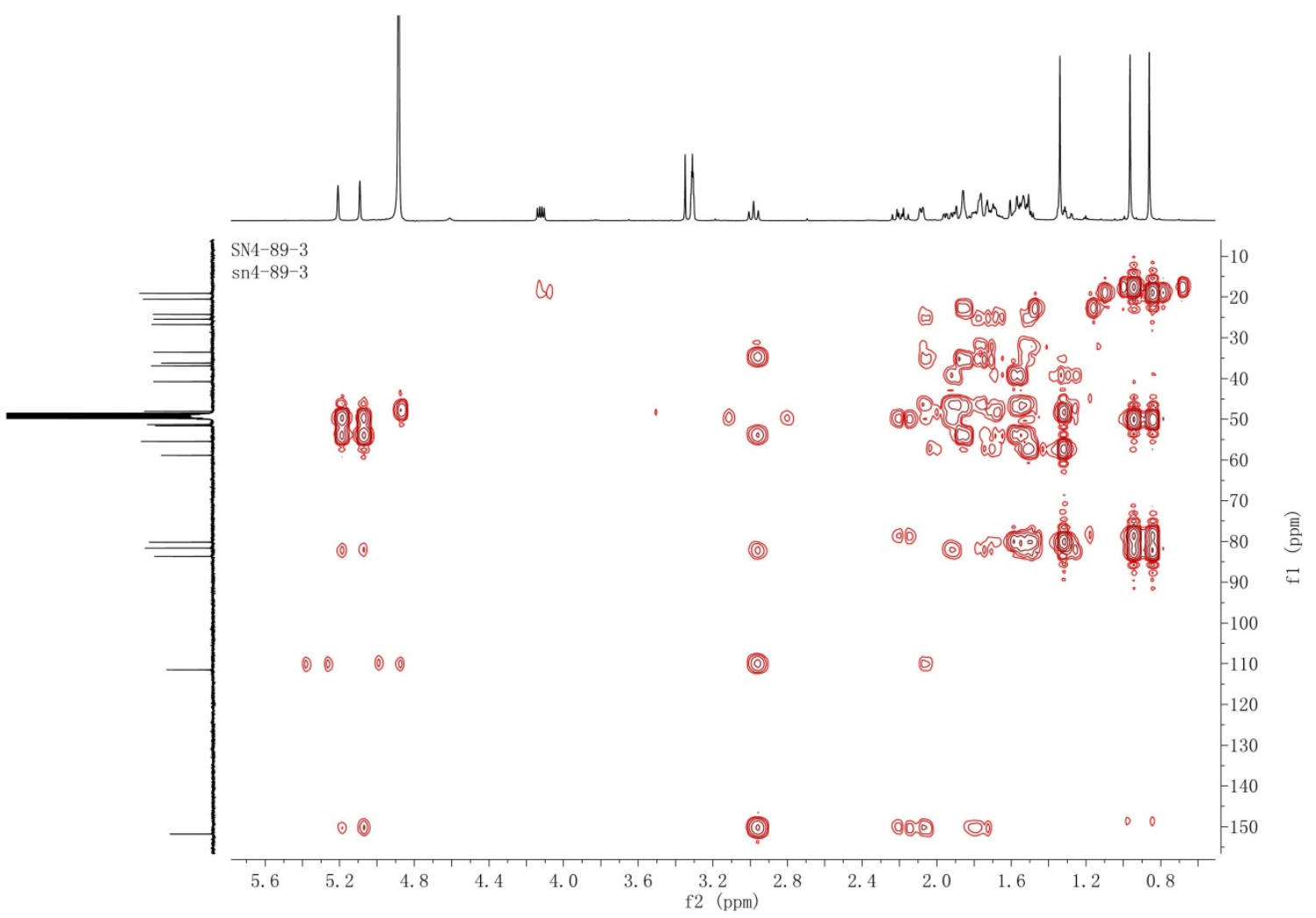

Figure S49. HMBC (methanol- $\left.d_{4}\right)$ spectrum of compound $5\left({ }^{1} \mathrm{H}: 400 \mathrm{MHz},{ }^{13} \mathrm{C}: 100 \mathrm{MHz}\right)$ 


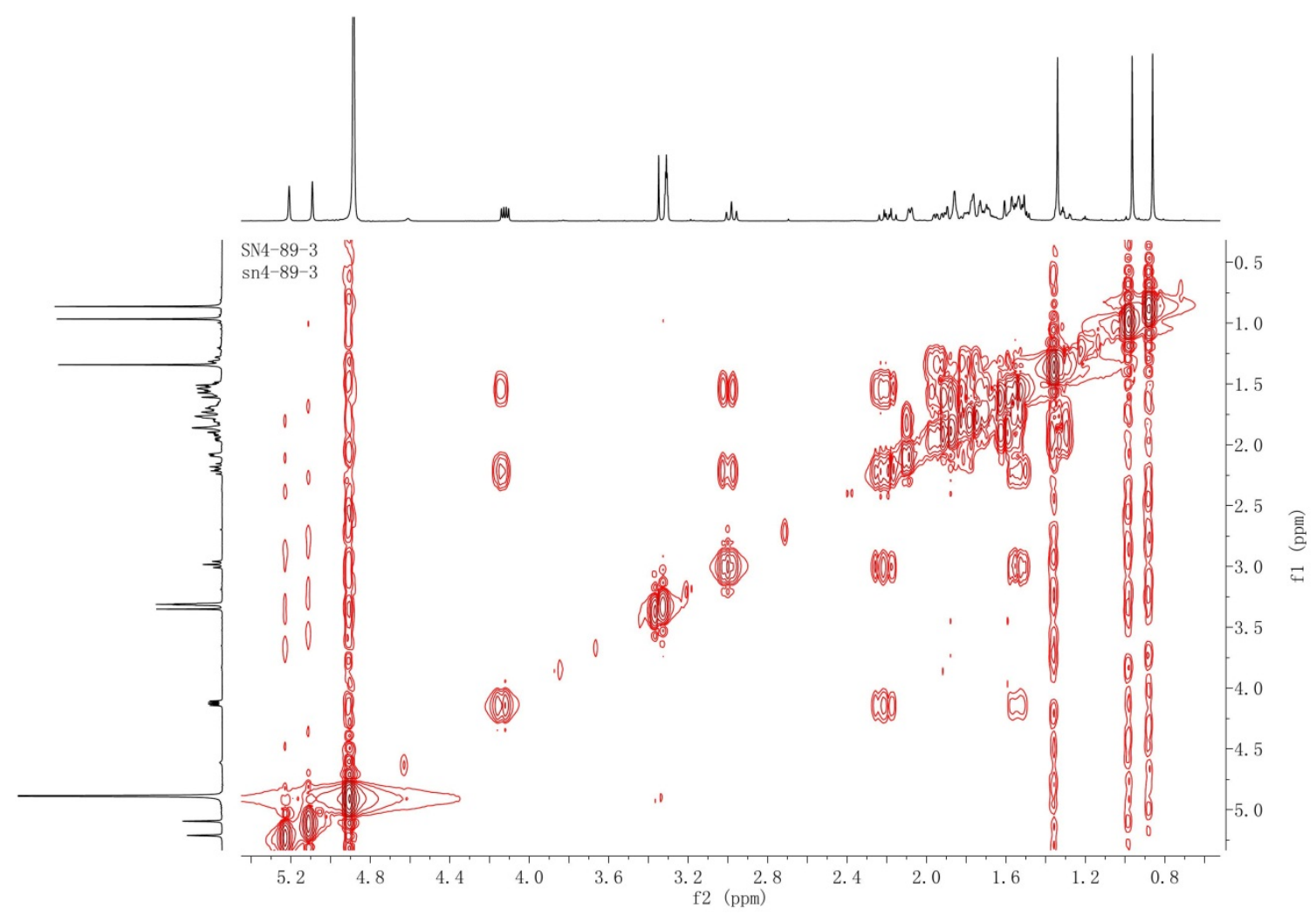

Figure S50. ${ }^{1} \mathrm{H}-{ }^{1} \mathrm{H}$ COSY (400 MHz, methanol- $\left.d_{4}\right)$ spectrum of compound 5

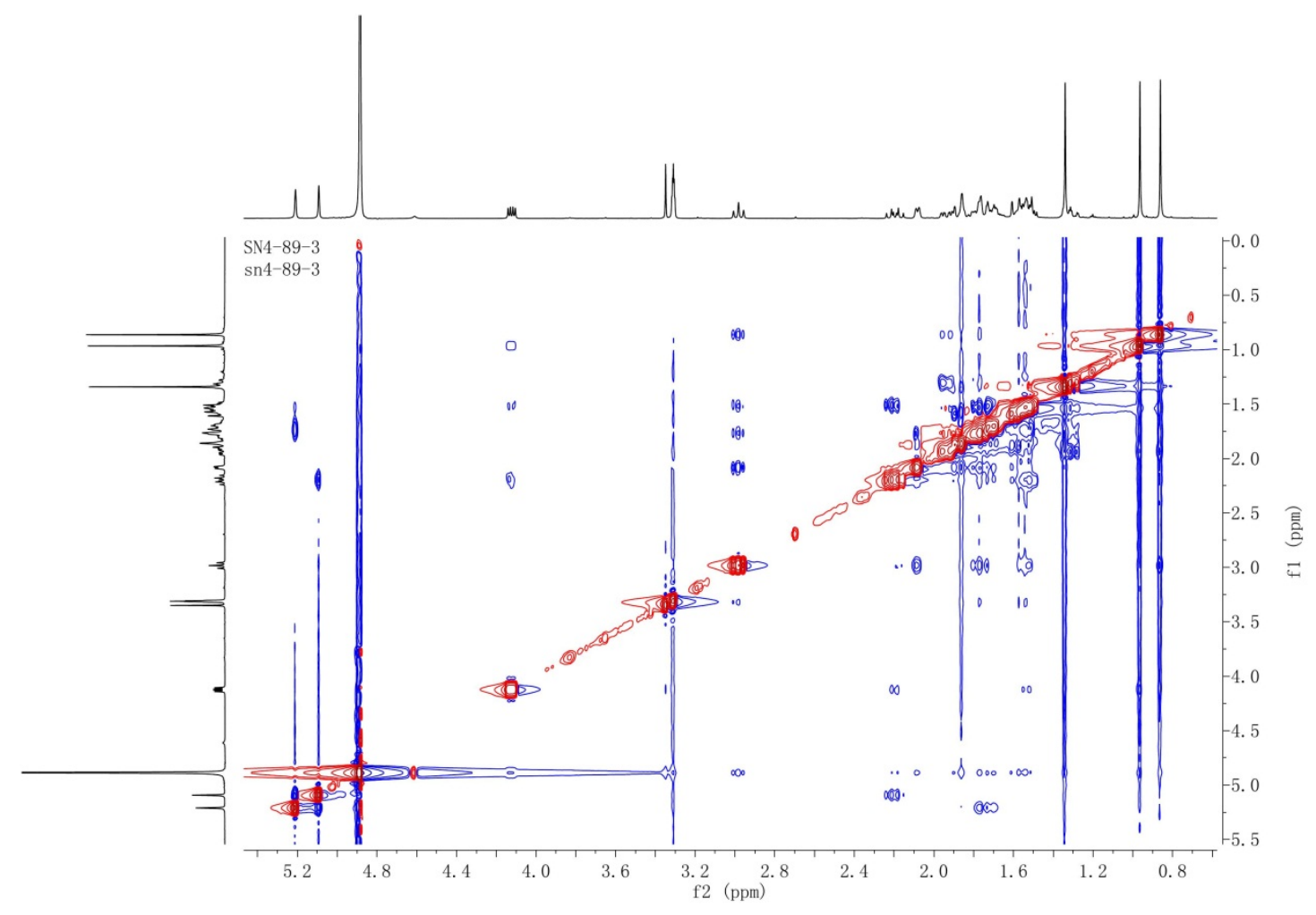

Figure S51. NOESY (400 MHz, methanol- $\left.d_{4}\right)$ spectrum of compound 5 


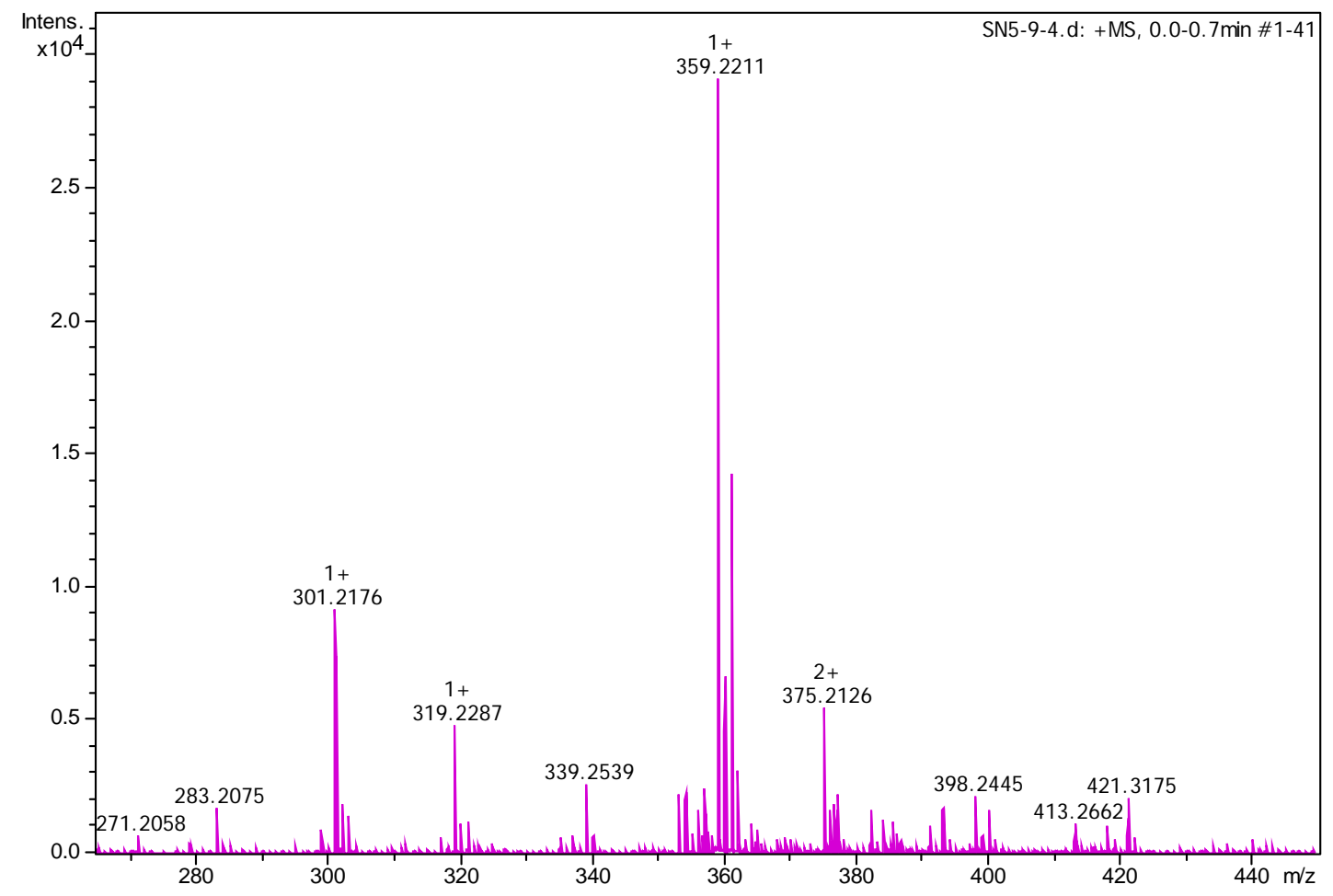

Figure S52. (+)-HRESIMS of compound 6

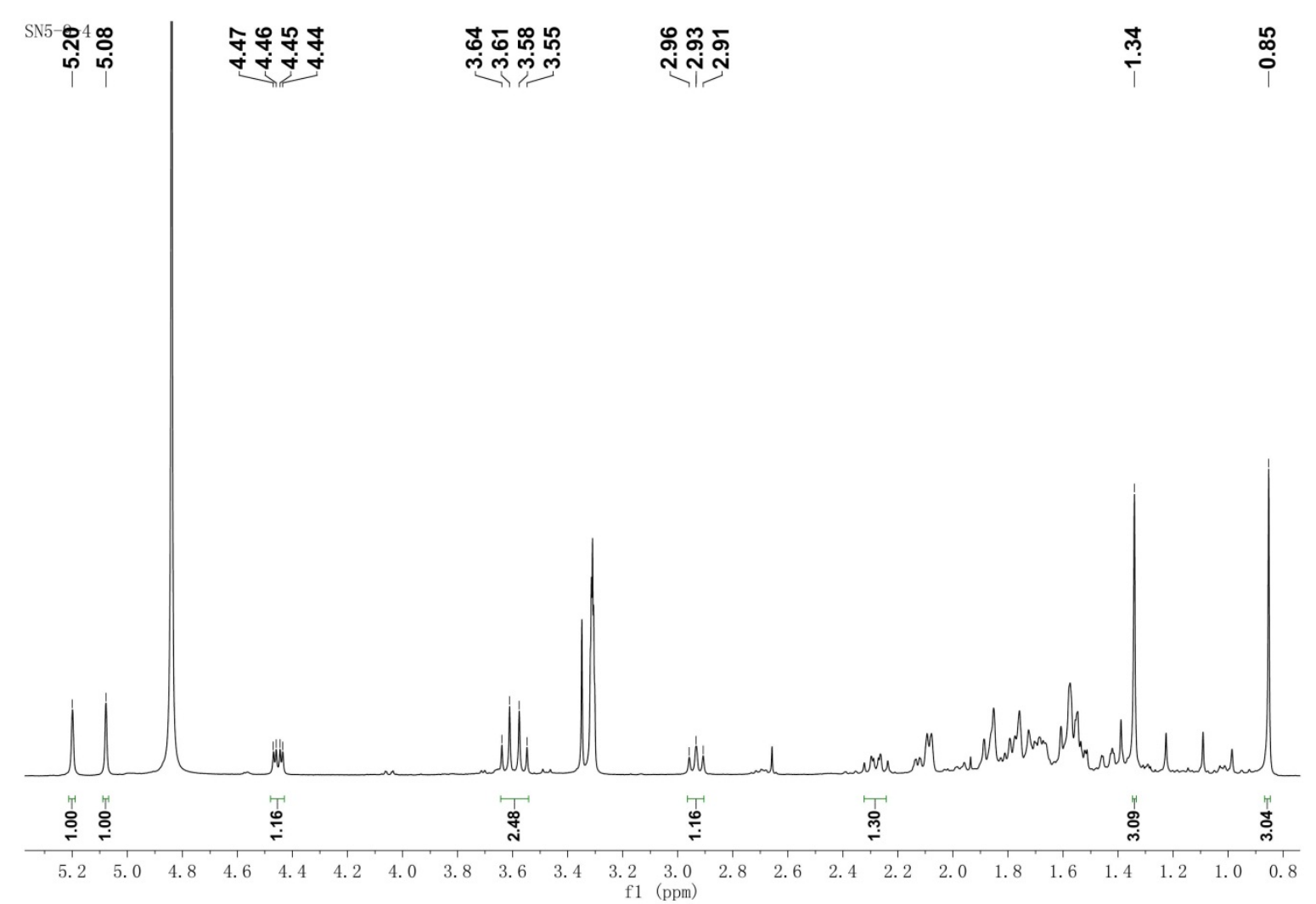

Figure S53. ${ }^{1} \mathrm{H}$ NMR (400 MHz, methanol- $d_{4}$ ) spectrum of compound 6 


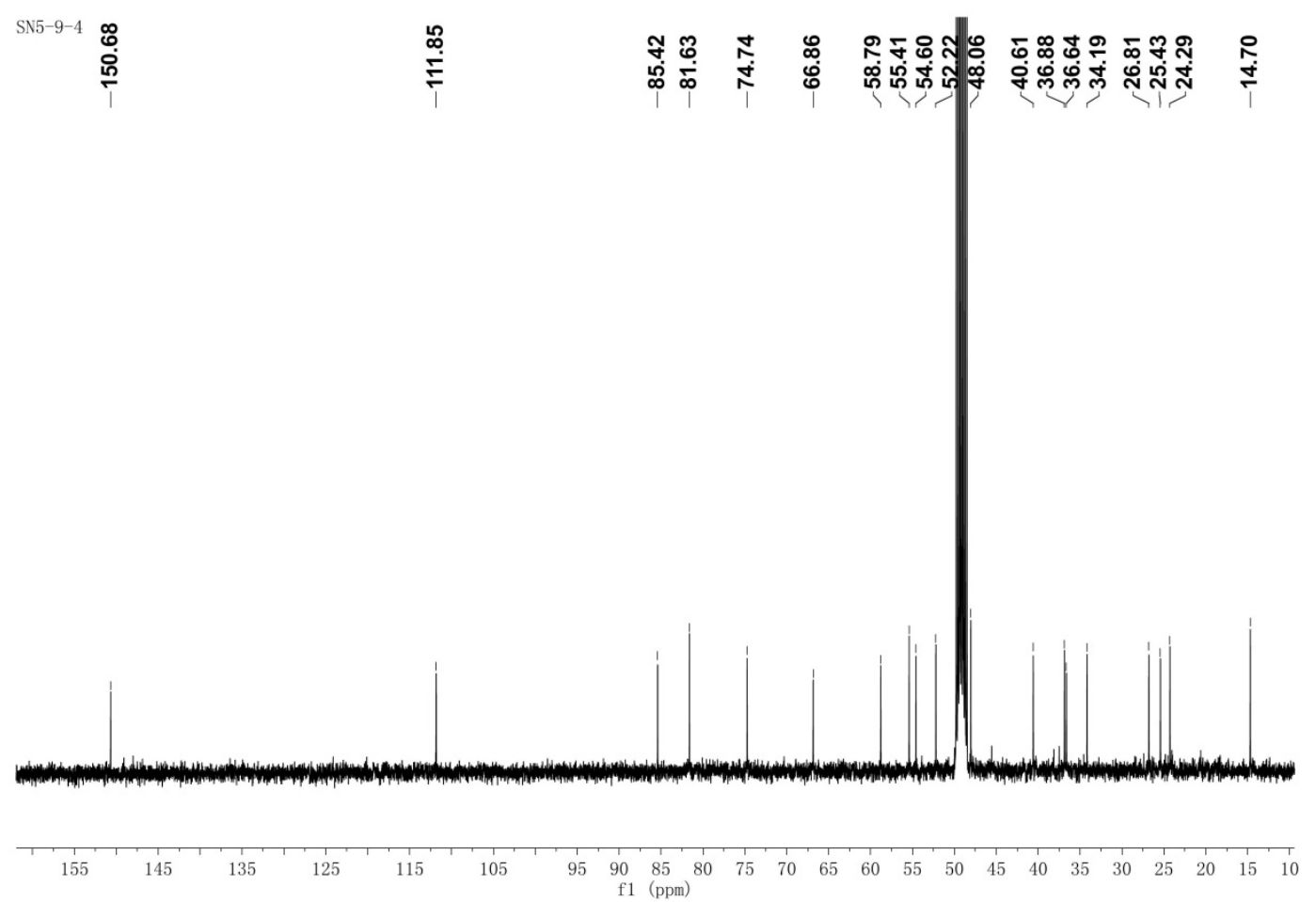

Figure S54. ${ }^{13} \mathrm{C}$ NMR $\left(100 \mathrm{MHz}\right.$, methanol- $\left.d_{4}\right)$ spectrum of compound 6

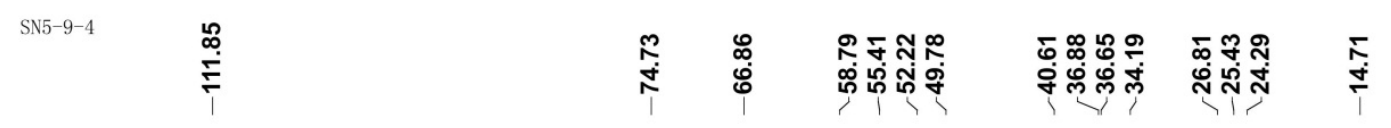

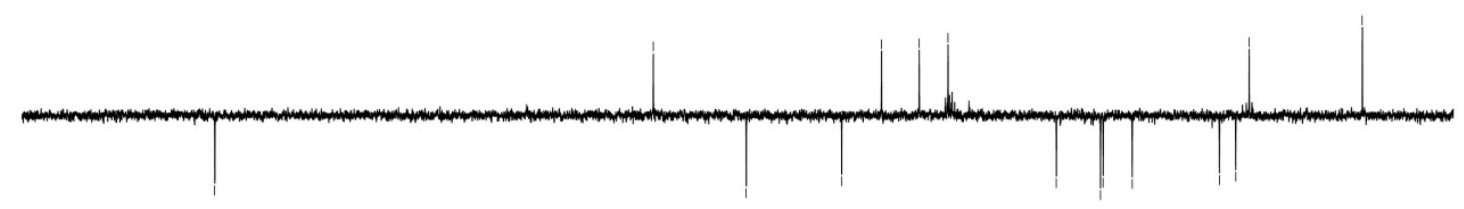

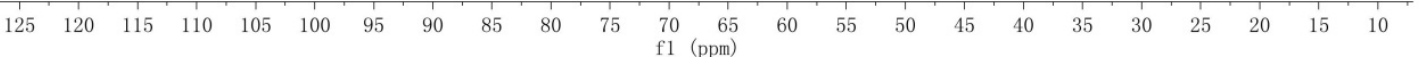

Figure S55. DEPT (100 MHz, methanol- $\left.d_{4}\right)$ spectrum of compound 6 


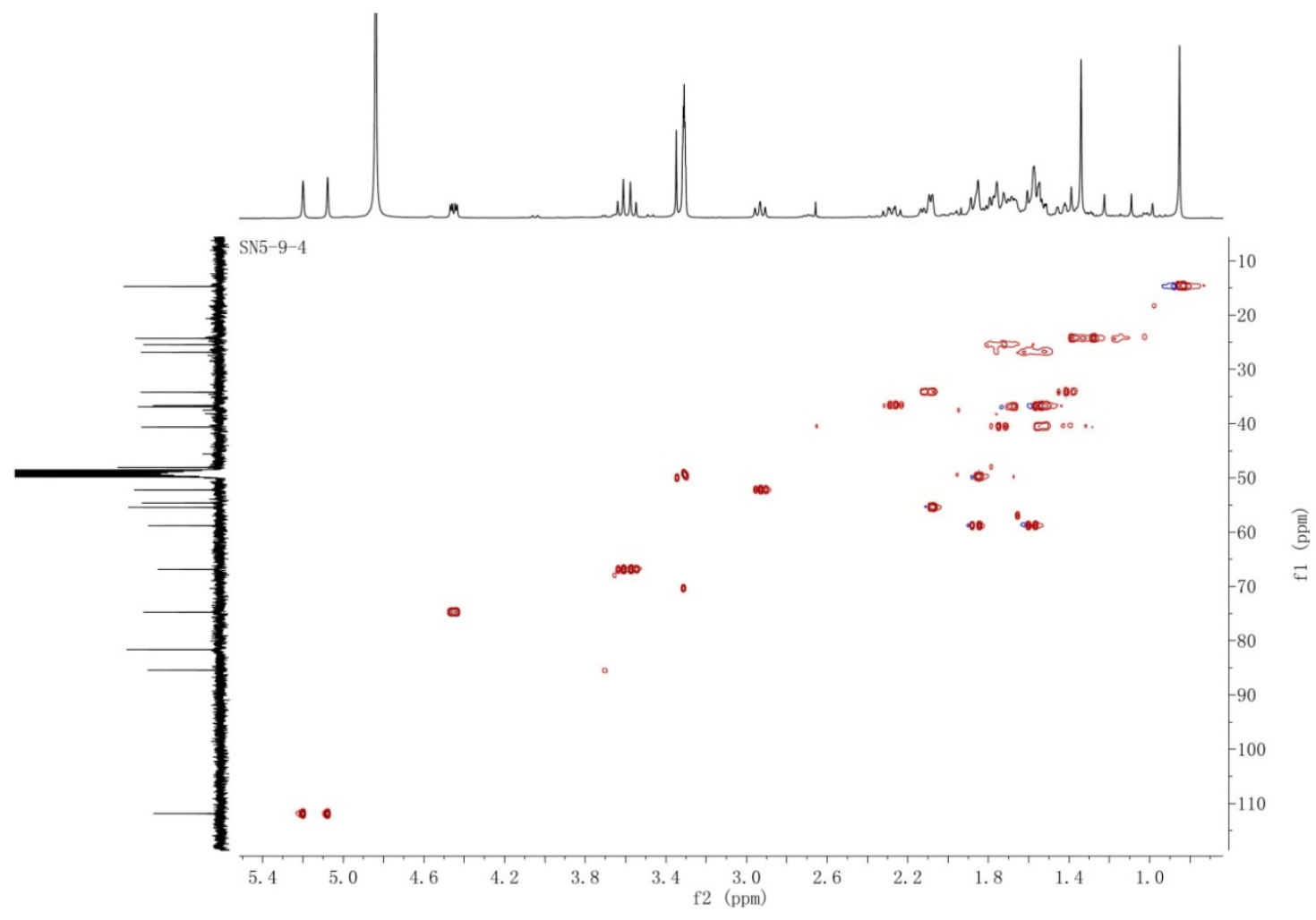

Figure S56. HSQC (methanol- $\left.d_{4}\right)$ spectrum of compound $6\left({ }^{1} \mathrm{H}: 400 \mathrm{MHz},{ }^{13} \mathrm{C}: 100 \mathrm{MHz}\right)$

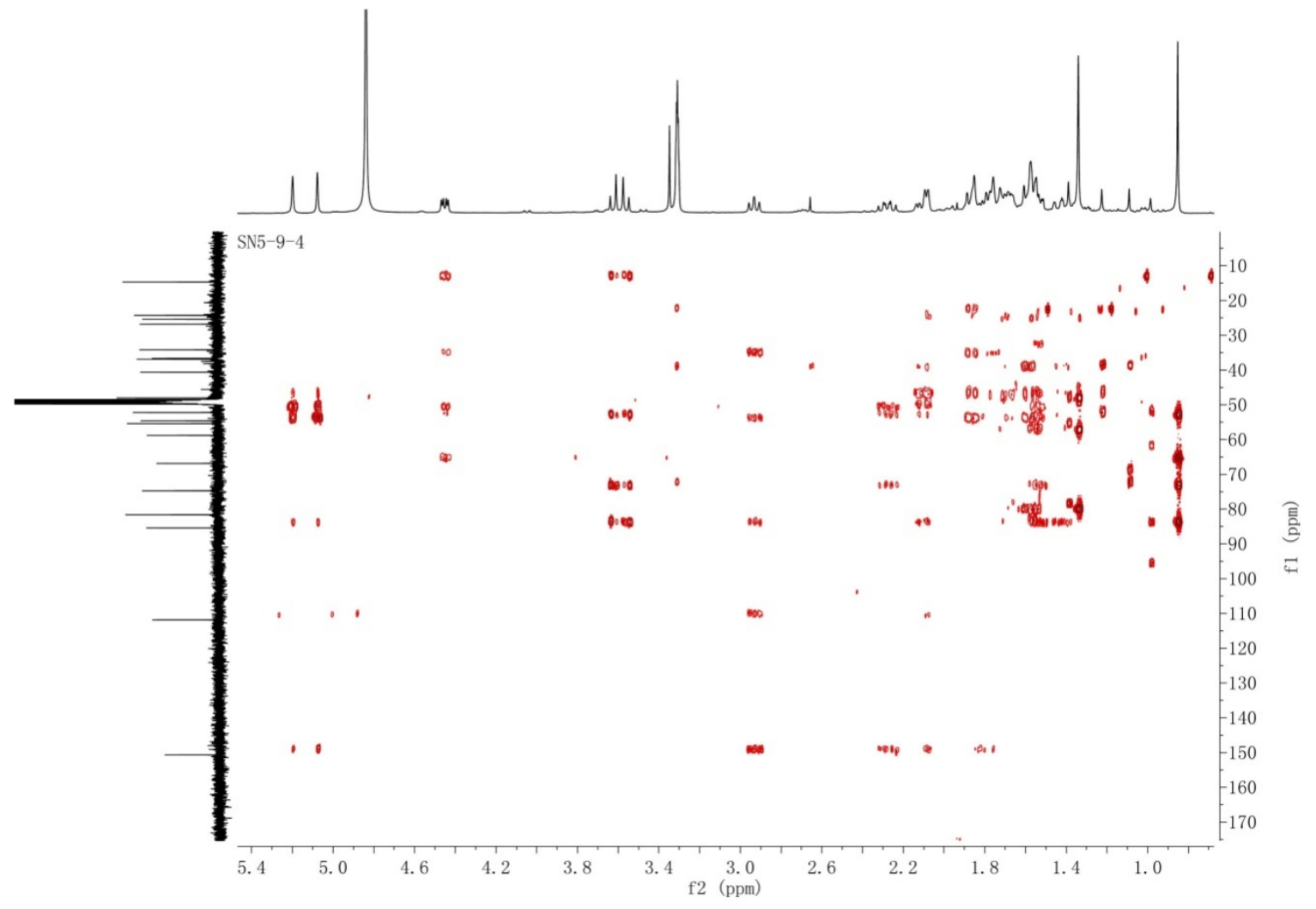

Figure S57. HMBC (methanol- $\left.d_{4}\right)$ spectrum of compound $6\left({ }^{1} \mathrm{H}: 400 \mathrm{MHz},{ }^{13} \mathrm{C}: 100 \mathrm{MHz}\right)$ 


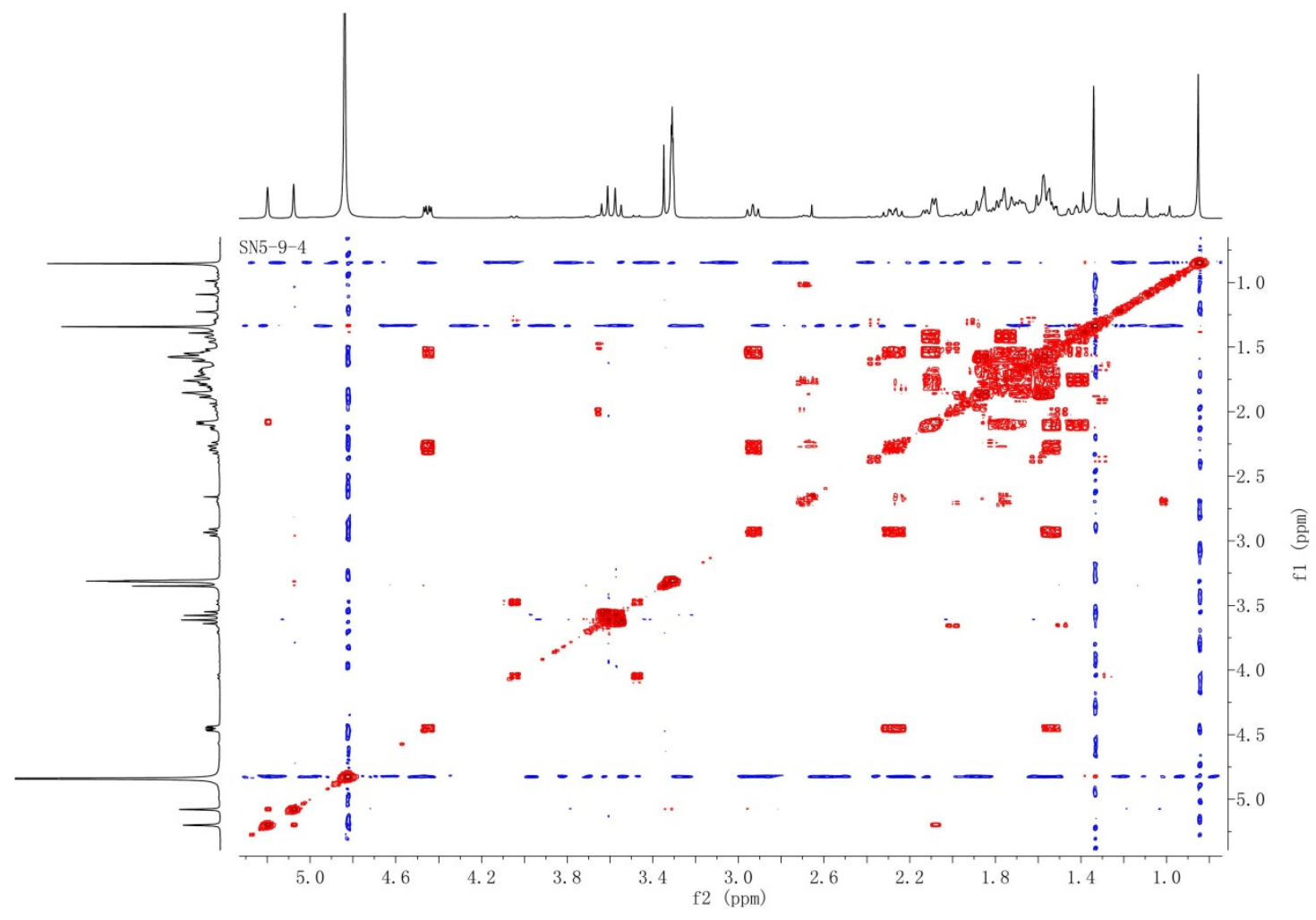

Figure S58. ${ }^{1} \mathrm{H}-{ }^{1} \mathrm{H}$ COSY (400 MHz, methanol- $\left.d_{4}\right)$ spectrum of compound 6

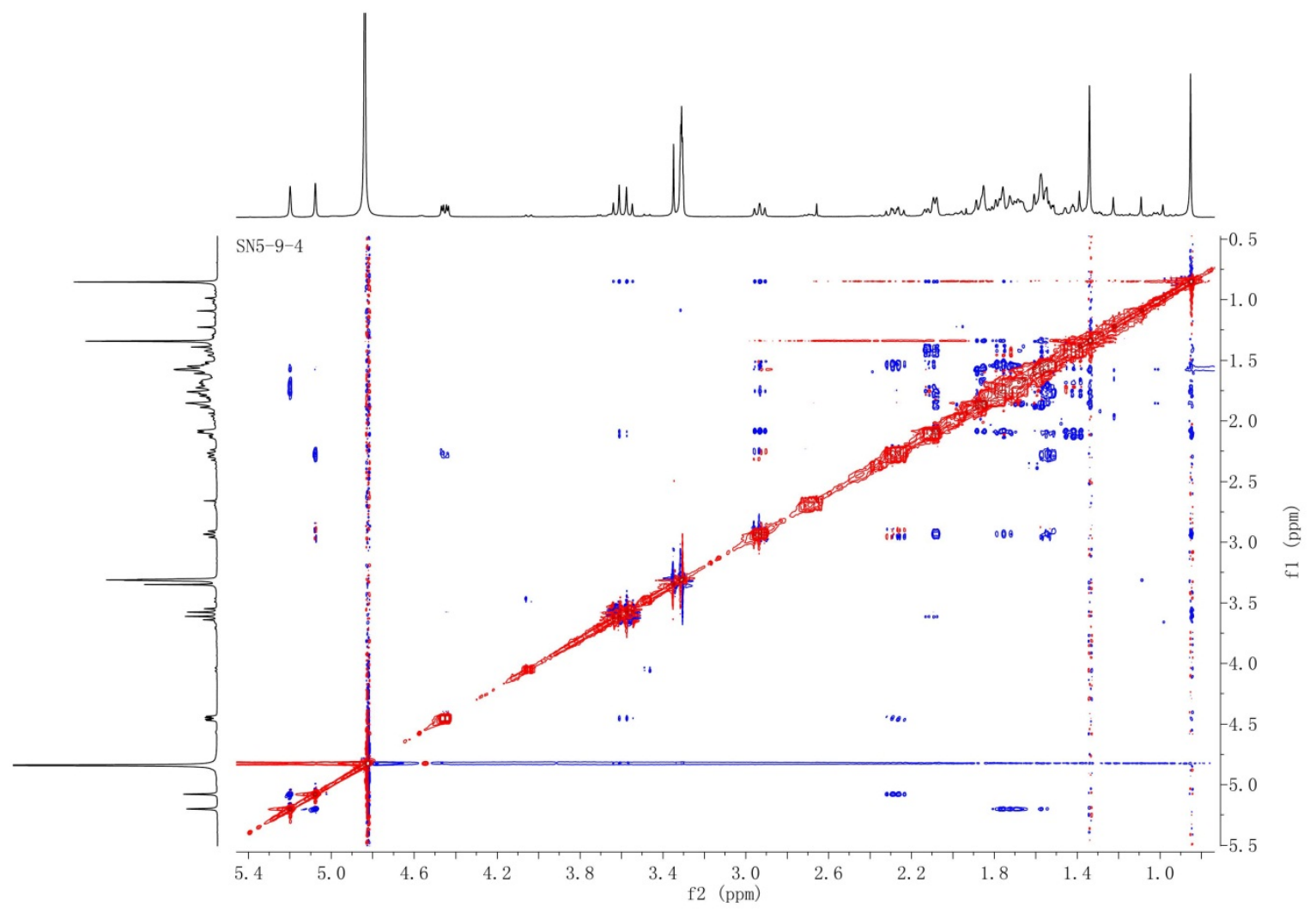

Figure S59. NOESY (400 MHz, methanol- $\left.d_{4}\right)$ spectrum of compound 6 


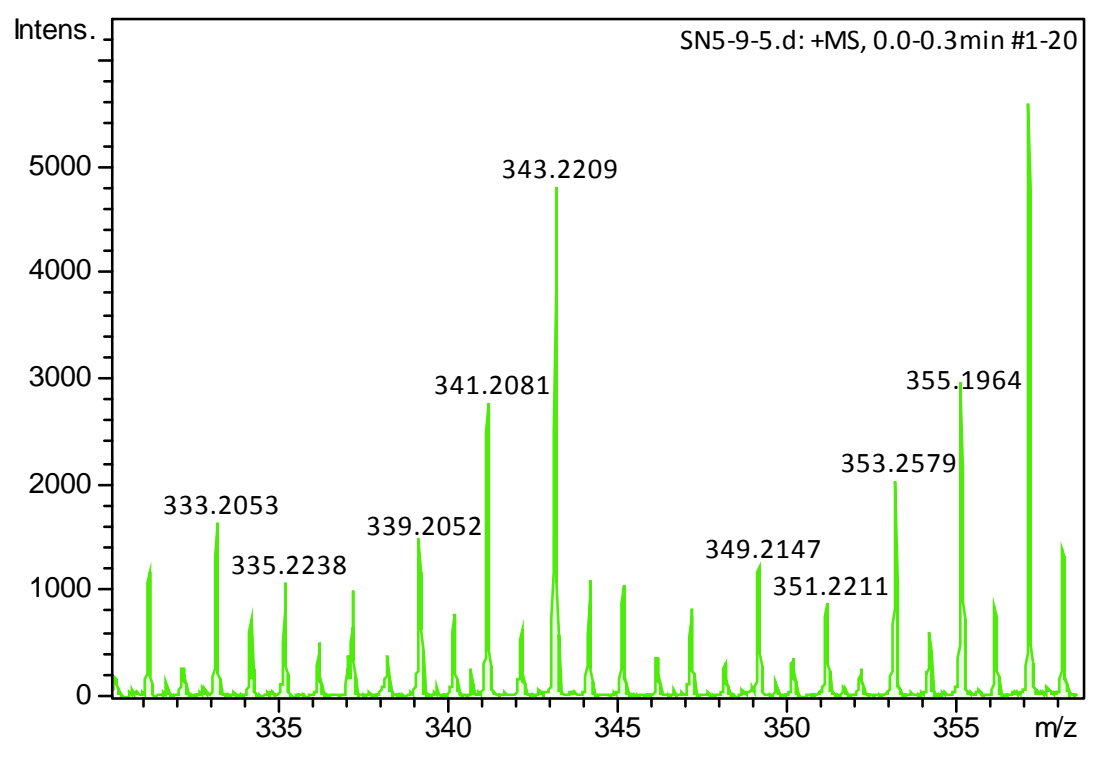

Figure S60. (+)-HRESIMS of compound 7

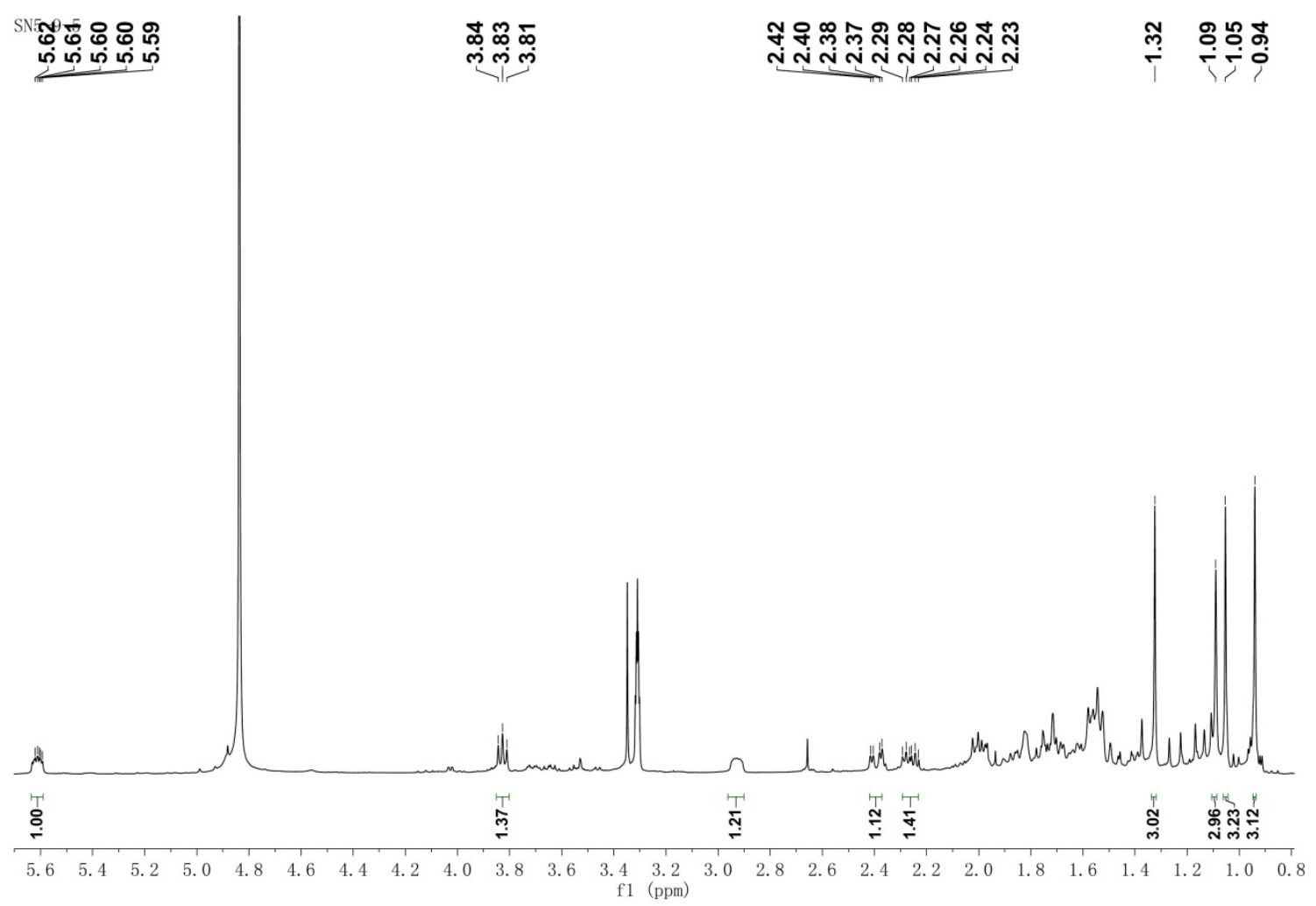

Figure S61. ${ }^{1} \mathrm{H}$ NMR (400 MHz, methanol- $d_{4}$ ) spectrum of compound 7 


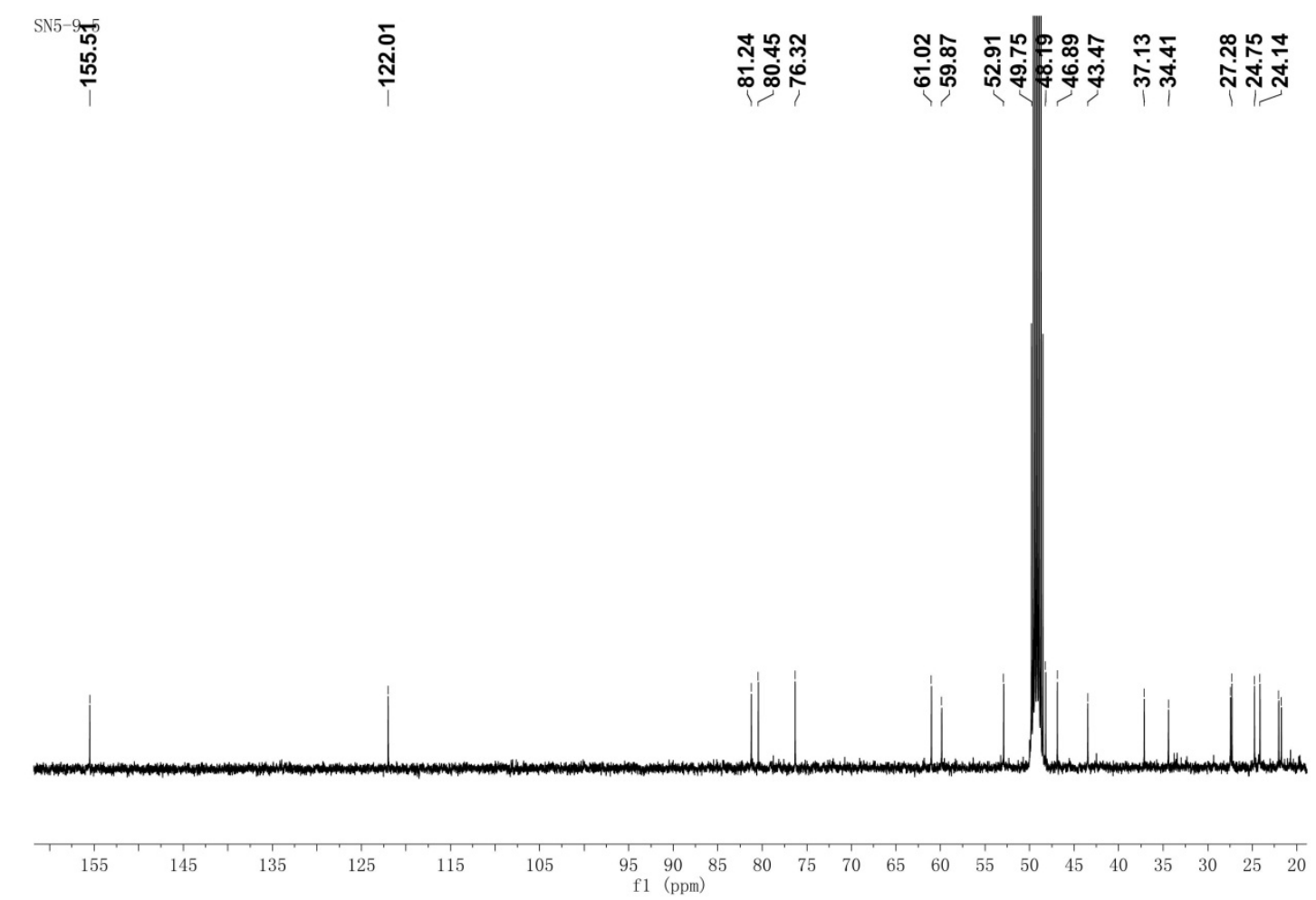

Figure S62. ${ }^{13} \mathrm{C}$ NMR $\left(100 \mathrm{MHz}\right.$, methanol- $\left.d_{4}\right)$ spectrum of compound 7

SN5-9-5

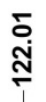

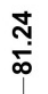

审䓦

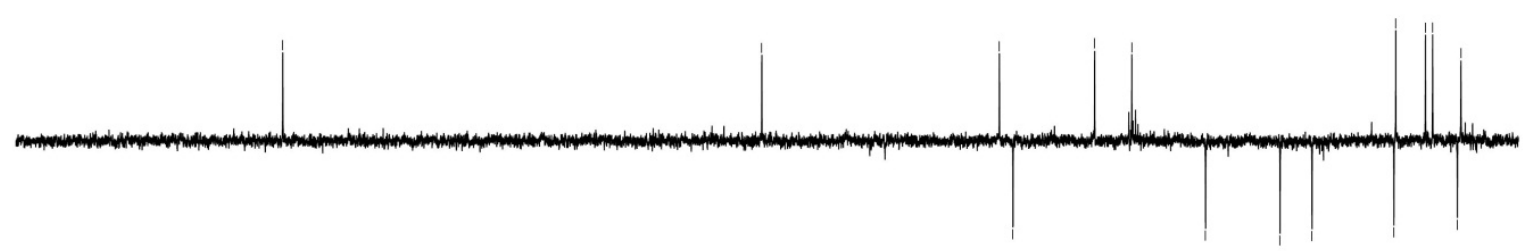

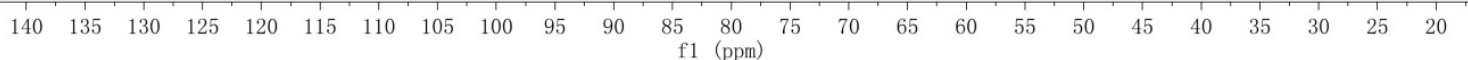

Figure S63. DEPT (100 MHz, methanol- $\left.d_{4}\right)$ spectrum of compound 7 


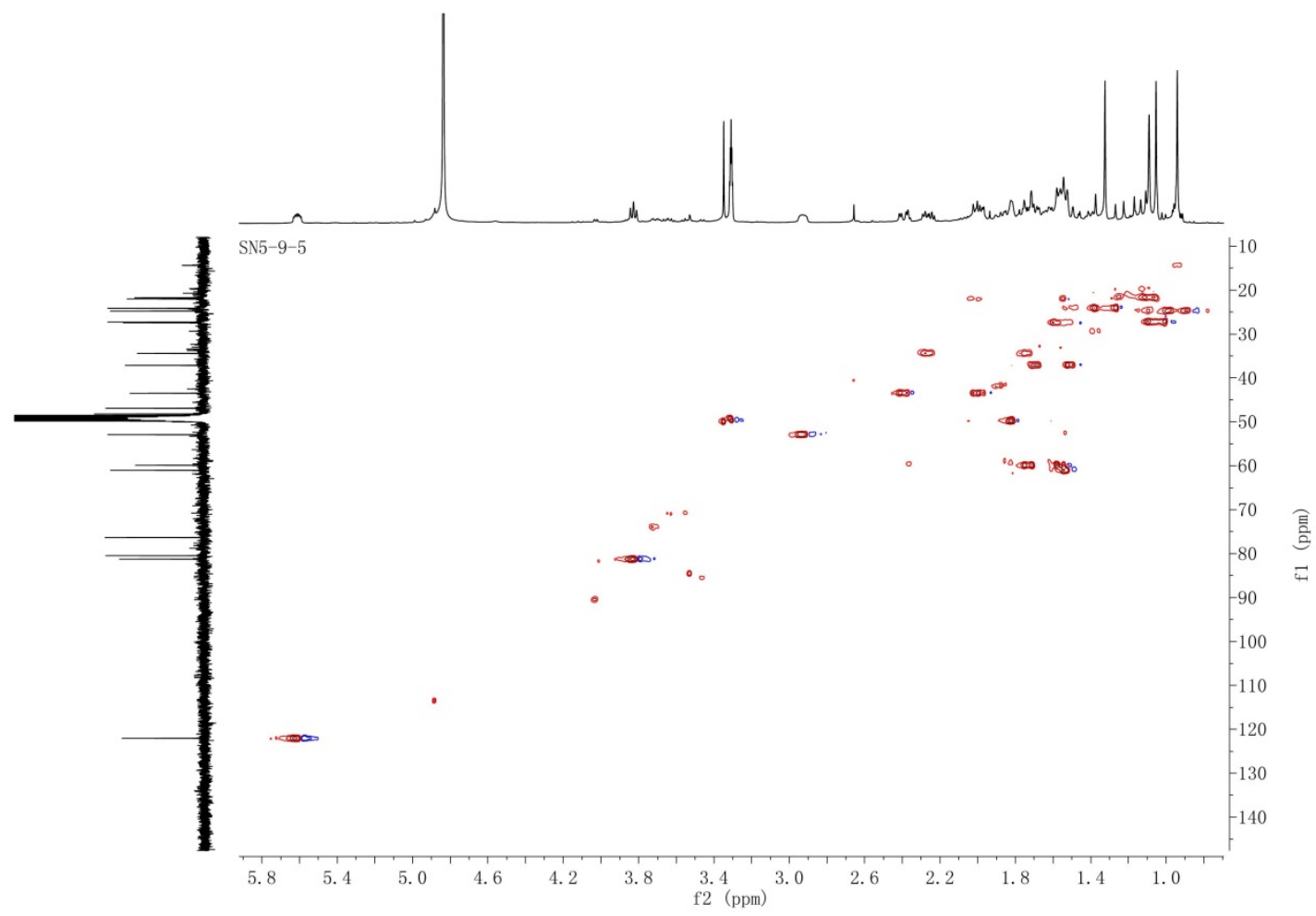

Figure S64. HSQC (methanol- $\left.d_{4}\right)$ spectrum of compound $7\left({ }^{1} \mathrm{H}: 400 \mathrm{MHz},{ }^{13} \mathrm{C}: 100 \mathrm{MHz}\right)$

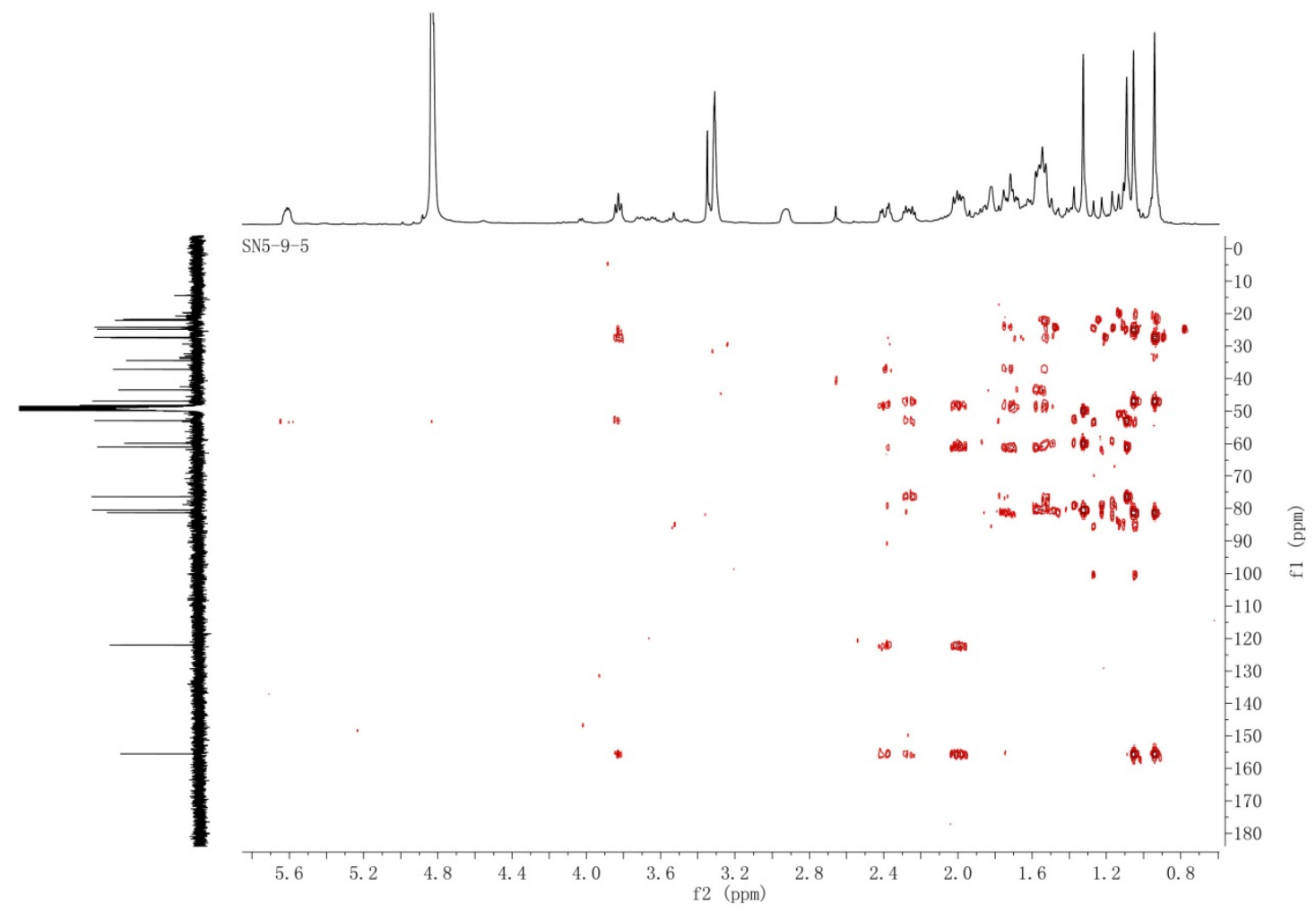

Figure S65. HMBC (methanol- $\left.d_{4}\right)$ spectrum of compound $7\left({ }^{1} \mathrm{H}: 400 \mathrm{MHz},{ }^{13} \mathrm{C}: 100 \mathrm{MHz}\right)$ 


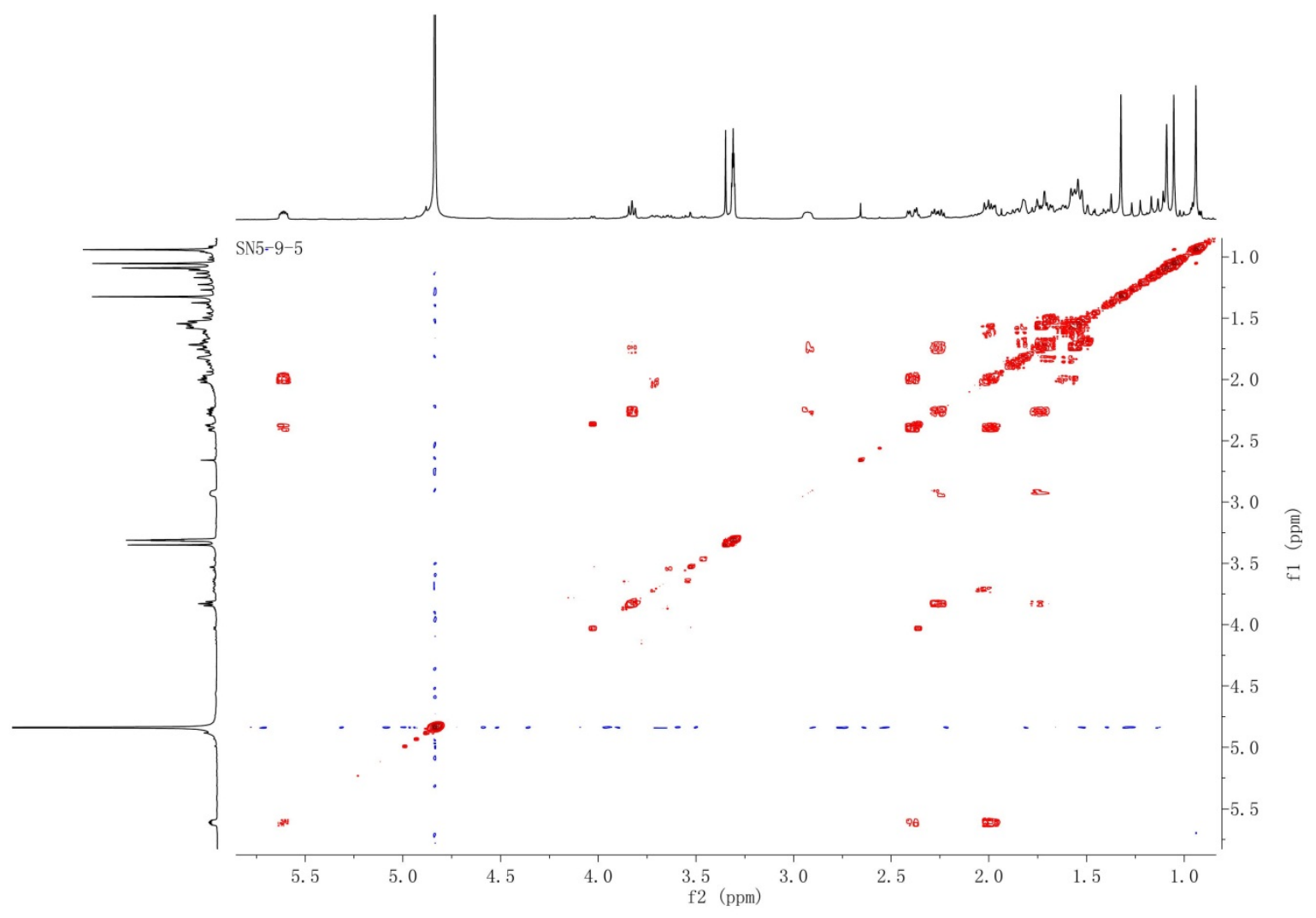

Figure S66. ${ }^{1} \mathrm{H}-{ }^{1} \mathrm{H}$ COSY $\left(400 \mathrm{MHz}\right.$, methanol- $\left.d_{4}\right)$ spectrum of compound 7

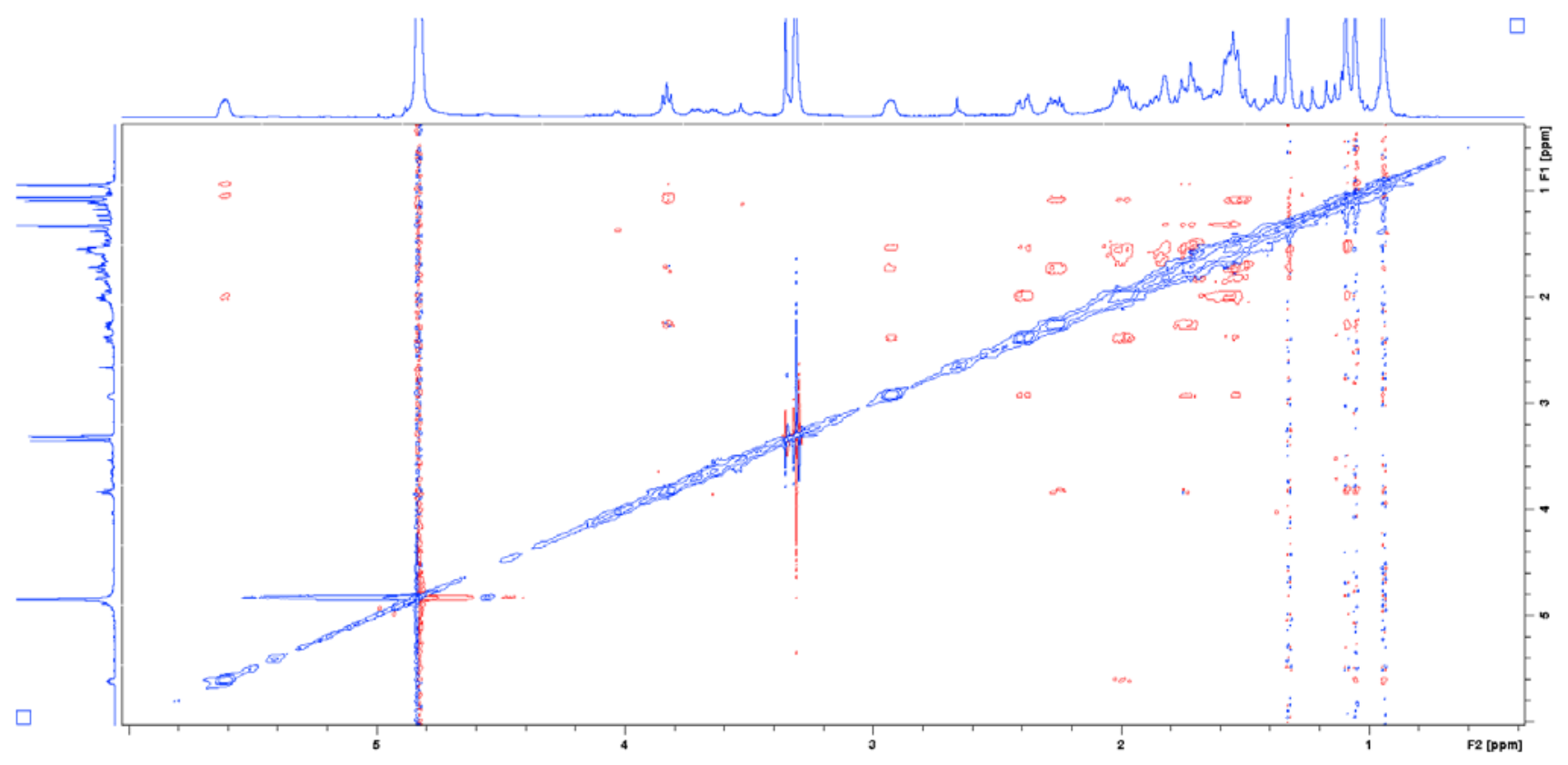

Figure S67. NOESY (400 MHz, methanol- $d_{4}$ ) spectrum of compound 7 


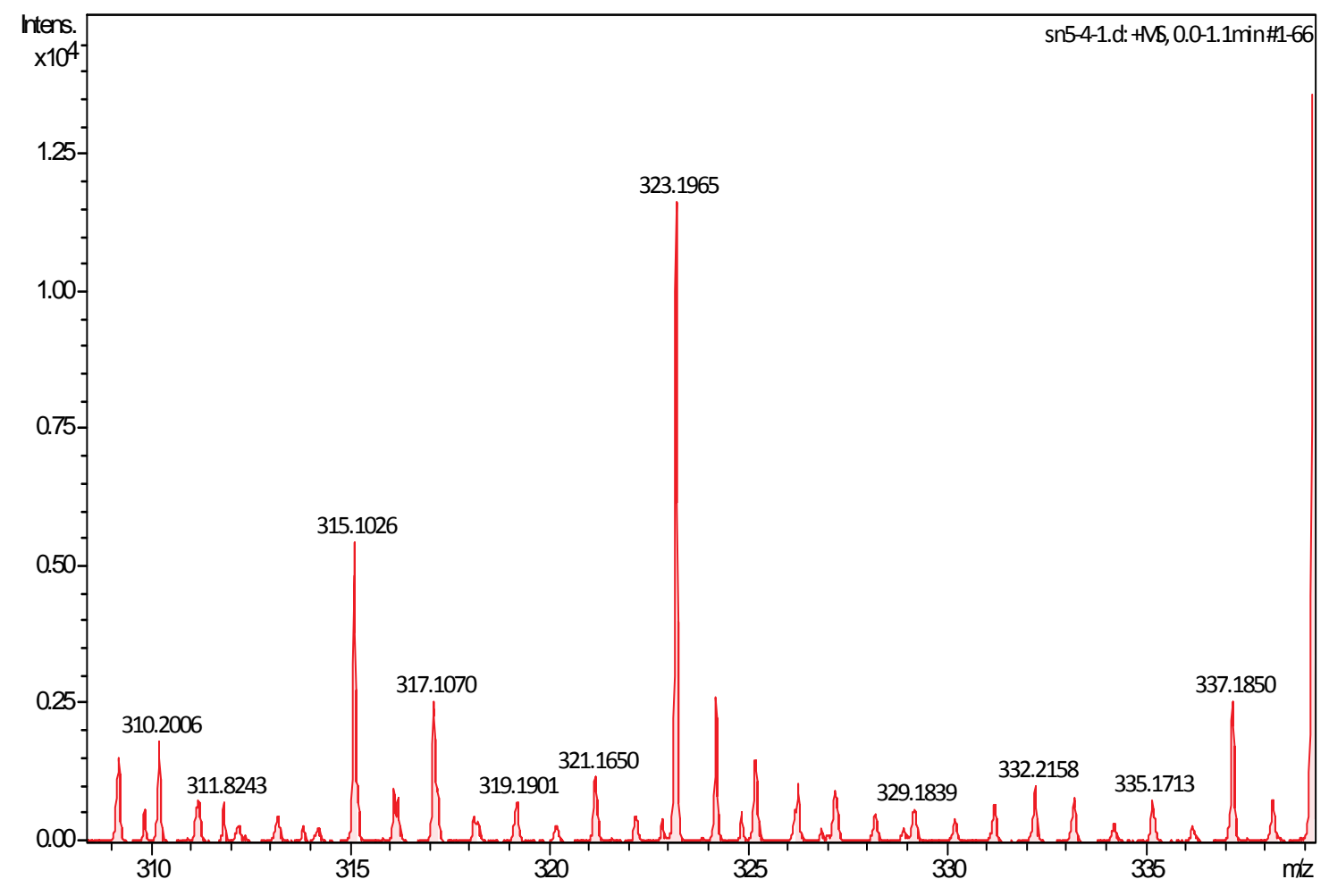

Figure S68. (+)-HRESIMS of compound 8

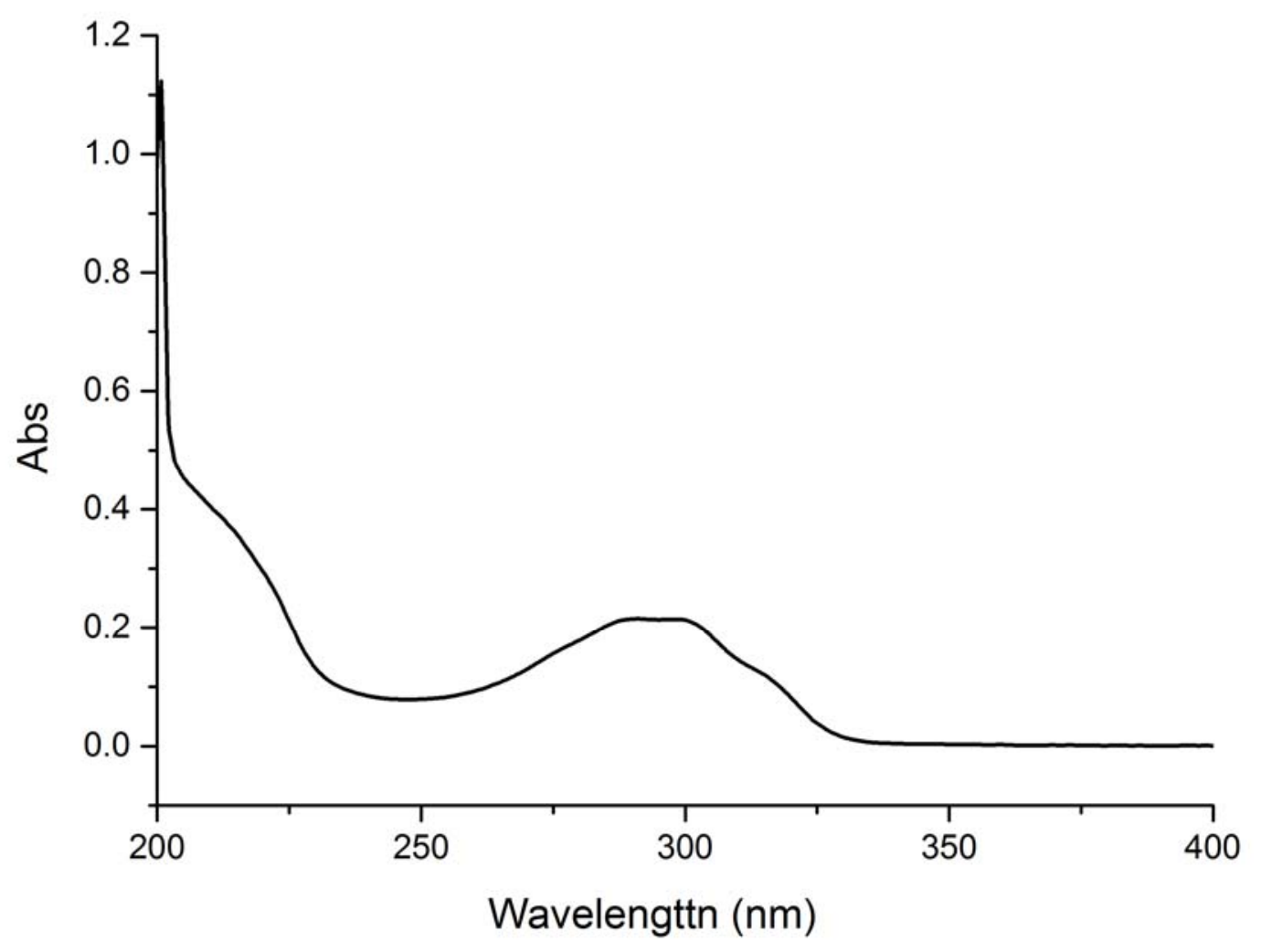

Figure S69. UV spectrum of compound 8 in $\mathrm{MeOH}$ 


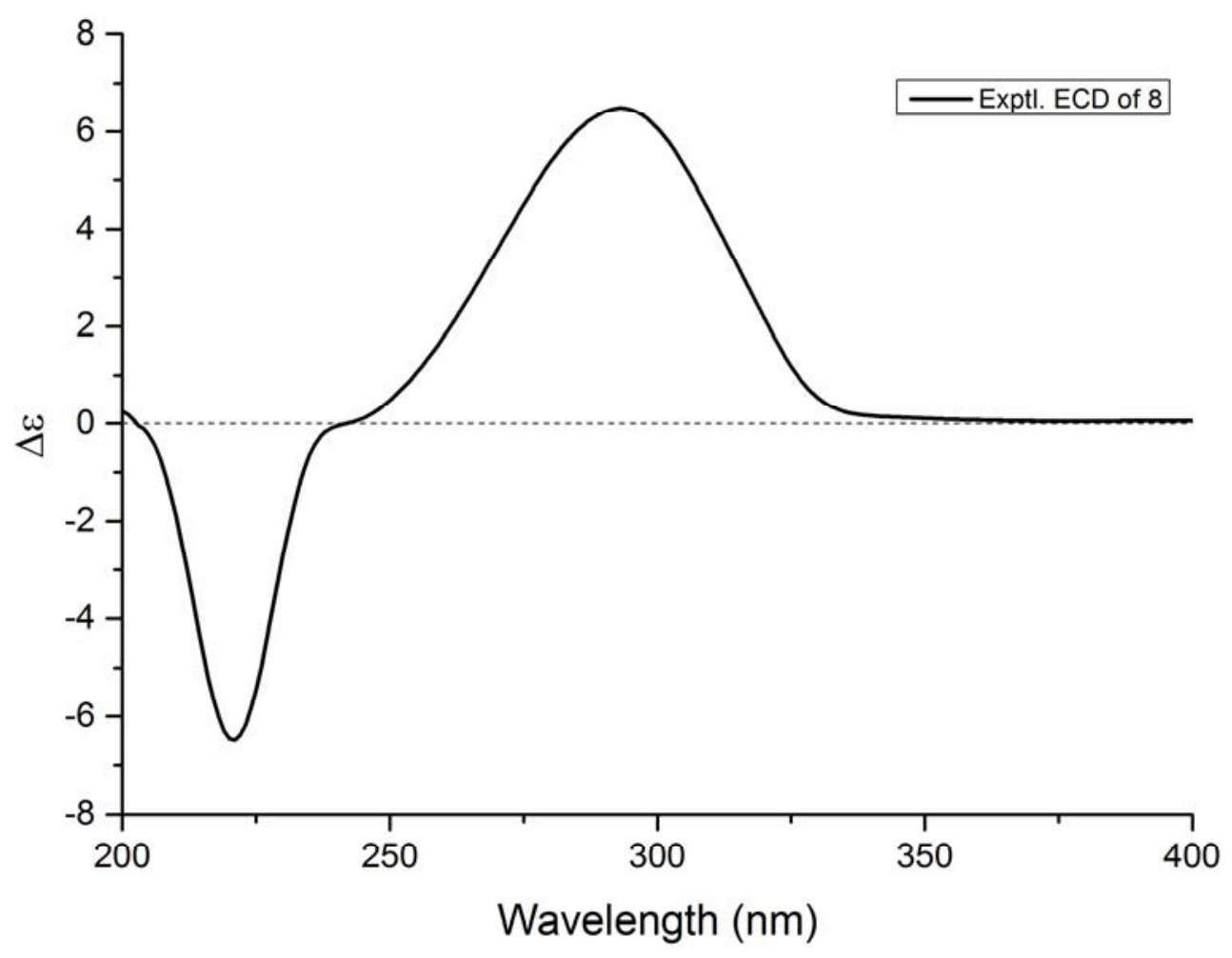

Figure S70. CD spectrum of compound 8 in $\mathrm{MeOH}$

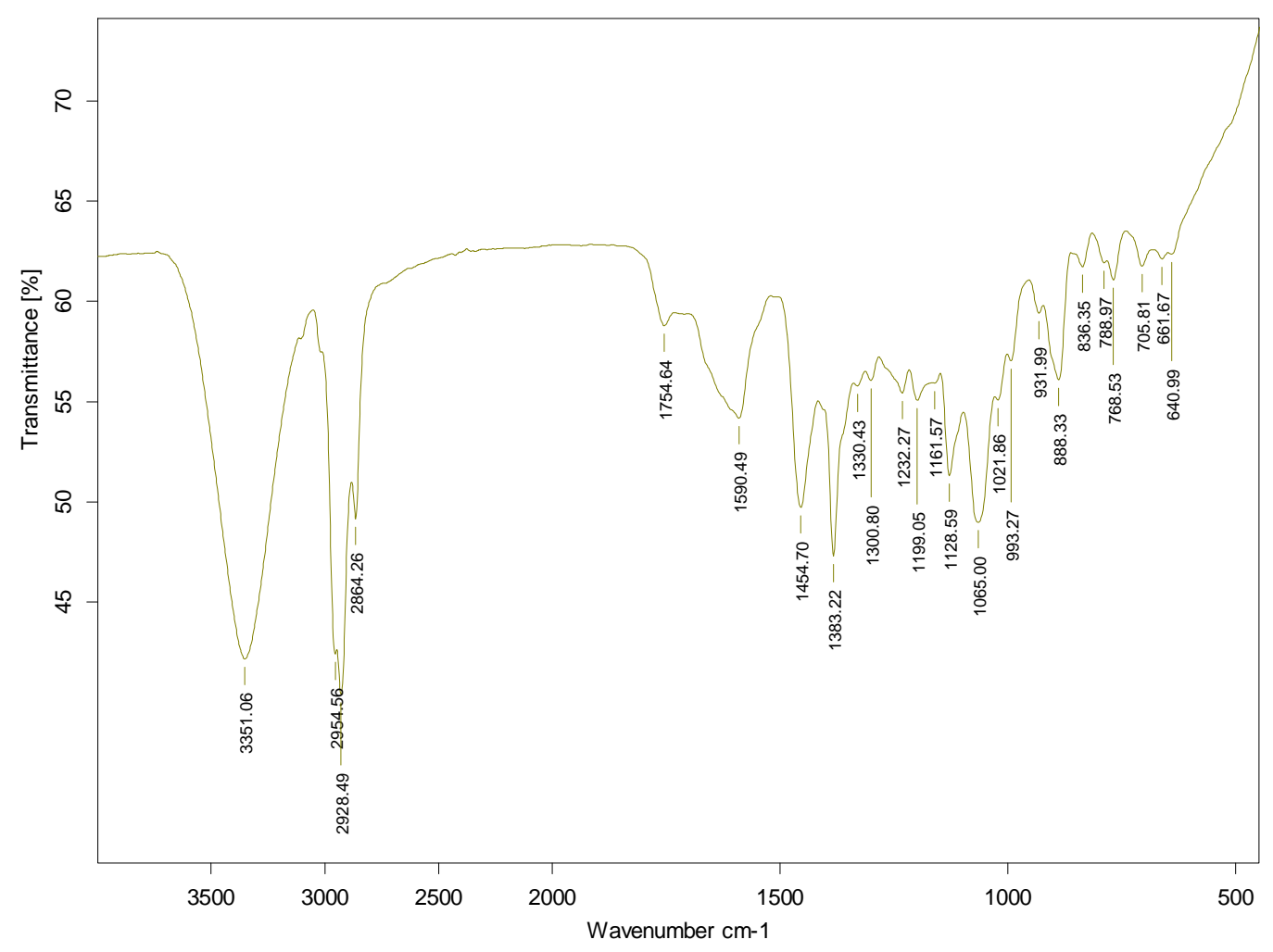

Figure S71. IR of compound 8 


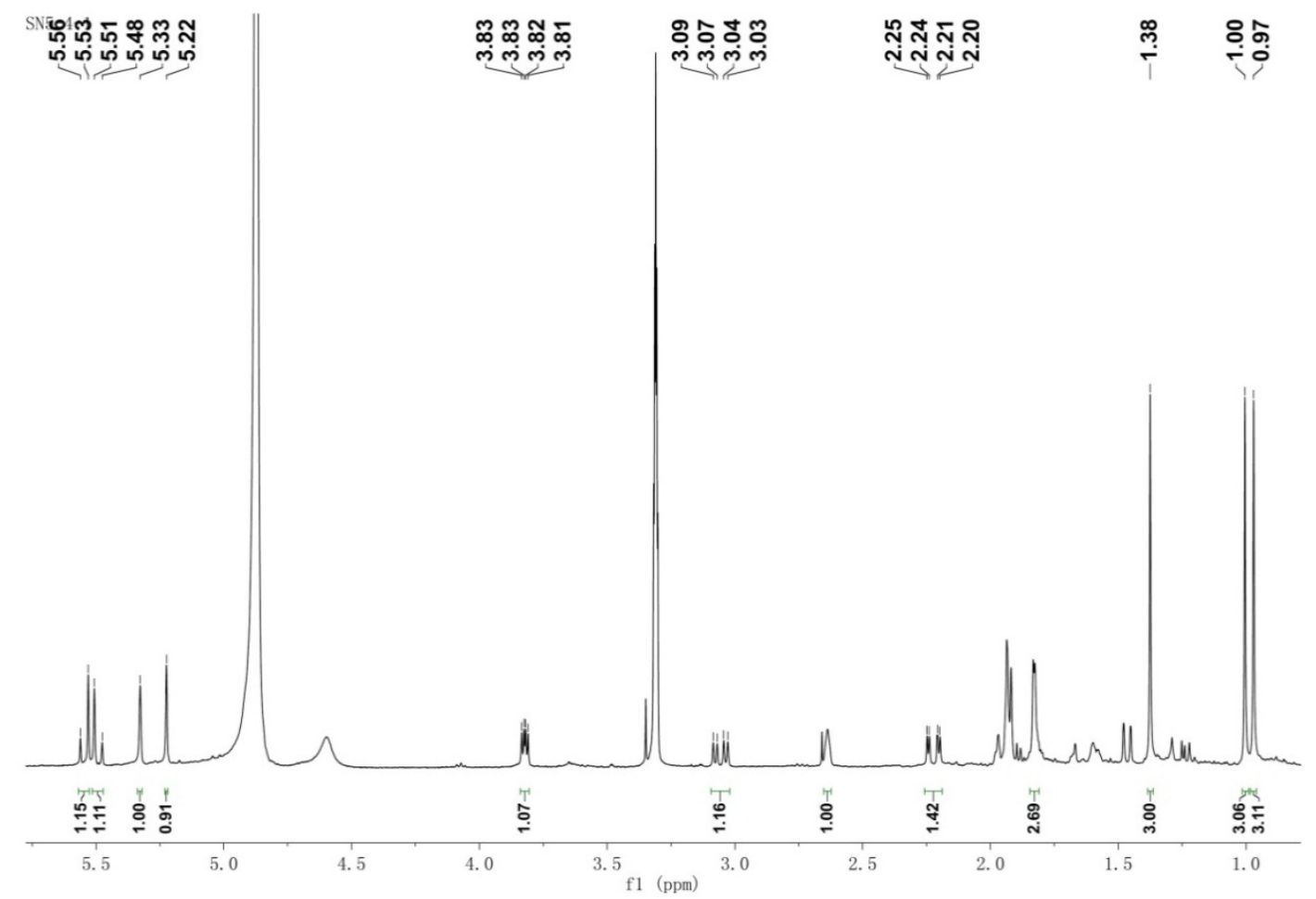

Figure S72. ${ }^{1} \mathrm{H}$ NMR (400 MHz, methanol- $d_{4}$ ) spectrum of compound 8
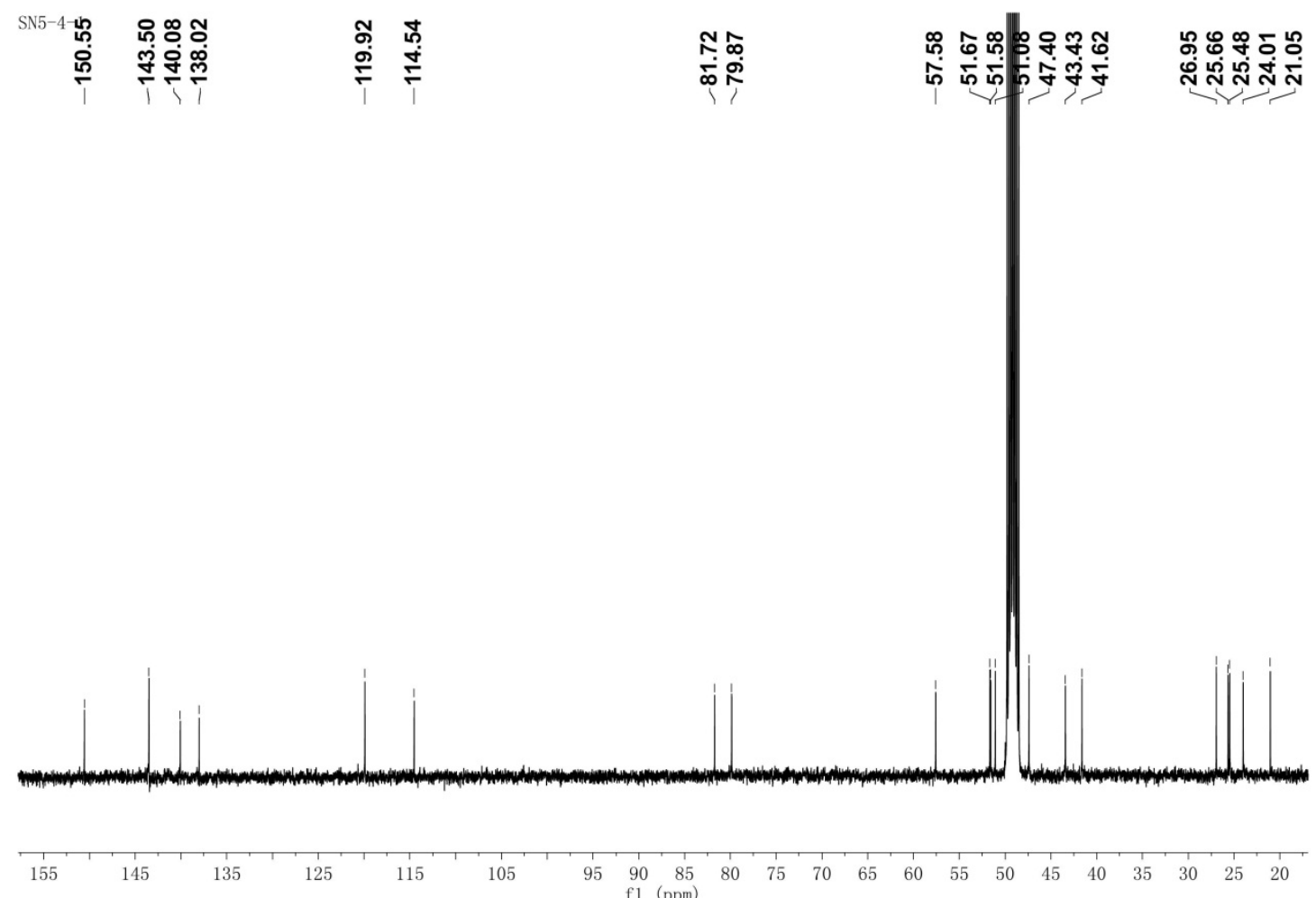

Figure S73. ${ }^{13} \mathrm{C}$ NMR (100 MHz, methanol- $\left.d_{4}\right)$ spectrum of compound 8 

SN5-4-1

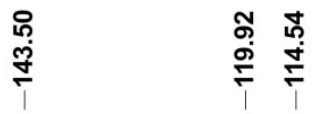

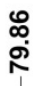

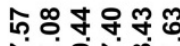

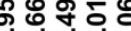

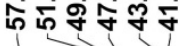

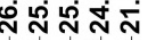

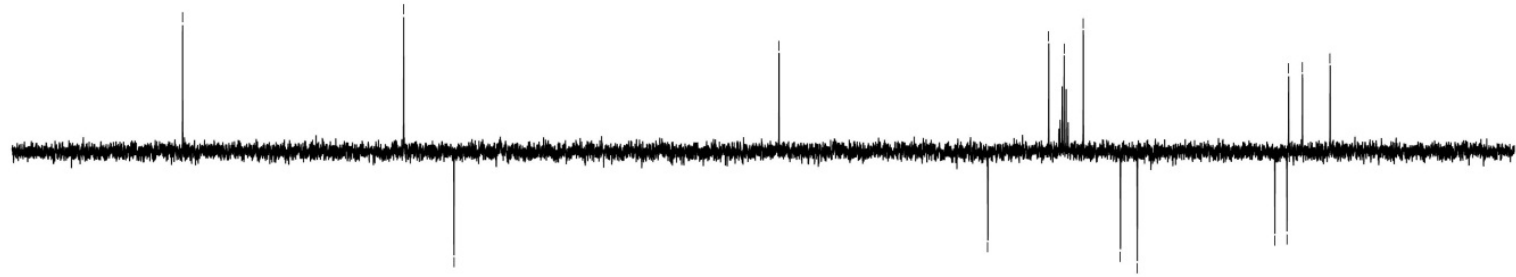

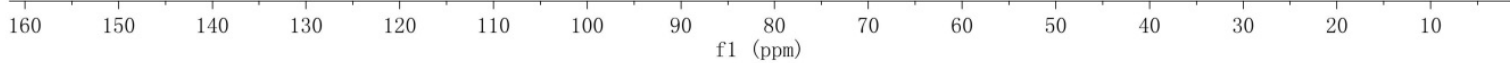

Figure S74. DEPT (100 MHz, methanol- $\left.d_{4}\right)$ spectrum of compound 8

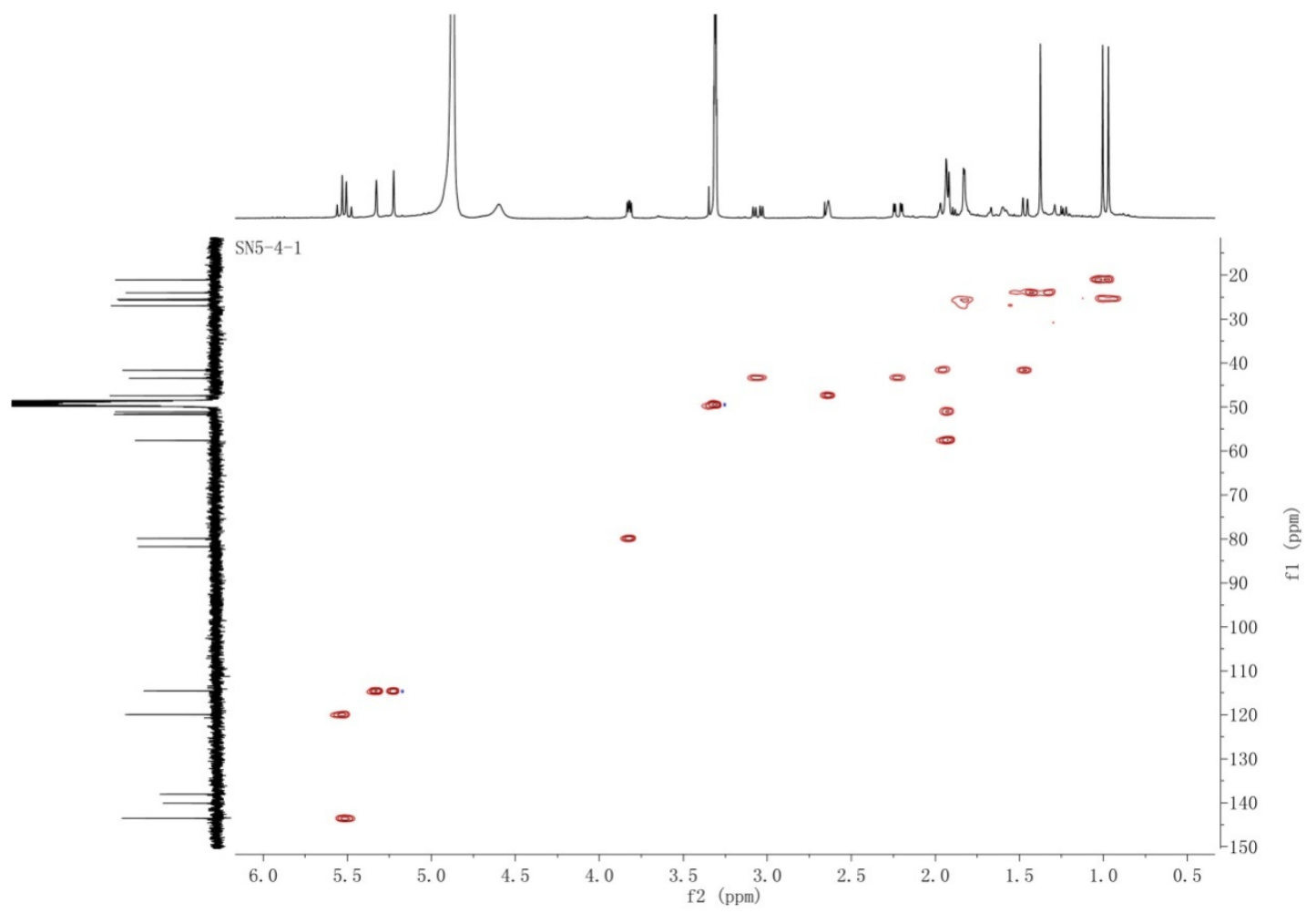

Figure S75. HSQC (methanol-d $\left.d_{4}\right)$ spectrum of compound $8\left({ }^{1} \mathrm{H}: 400 \mathrm{MHz},{ }^{13} \mathrm{C}: 100 \mathrm{MHz}\right)$ 


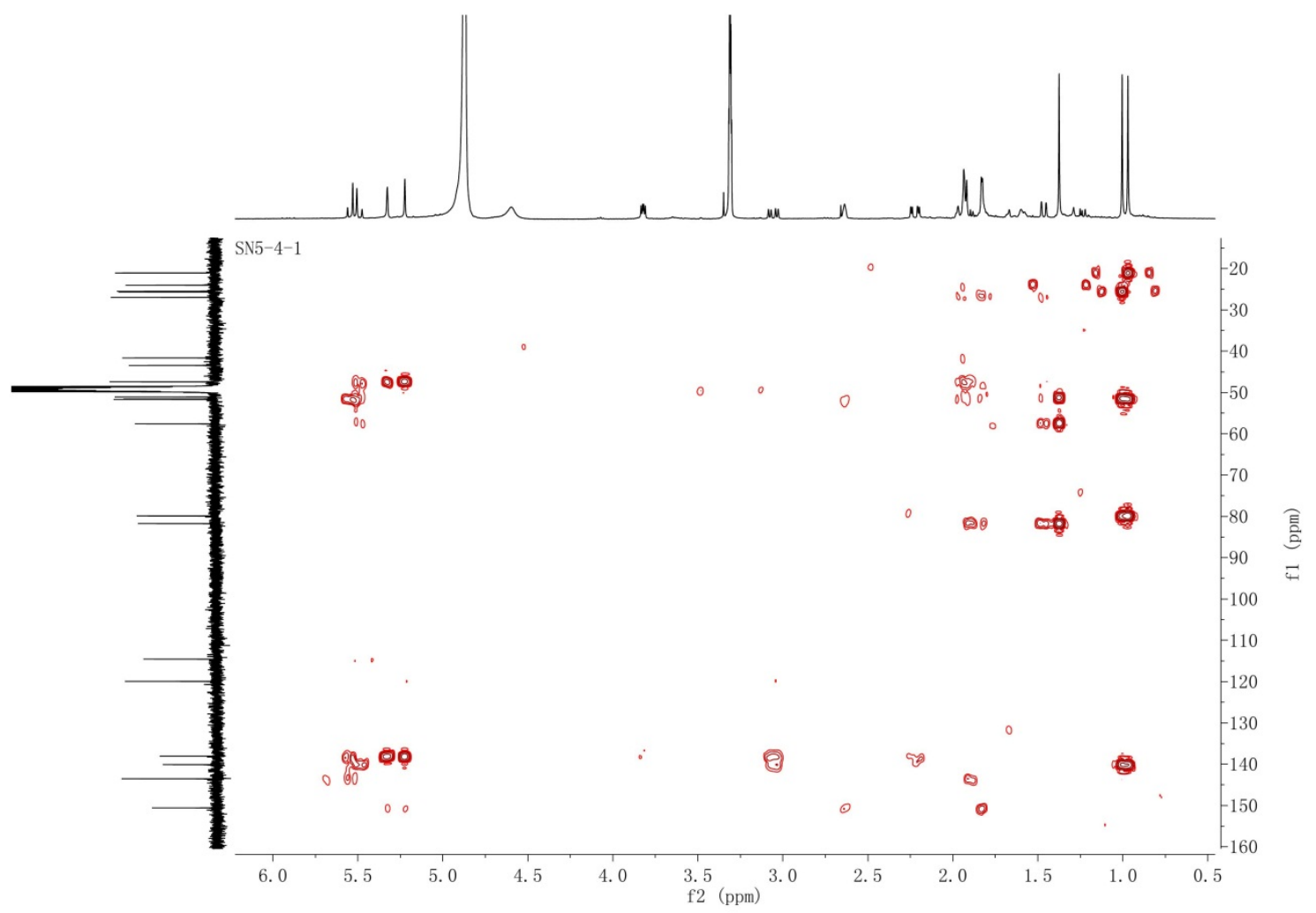

Figure S76. HMBC (methanol- $\left.d_{4}\right)$ spectrum of compound $8\left({ }^{1} \mathrm{H}: 400 \mathrm{MHz},{ }^{13} \mathrm{C}: 100 \mathrm{MHz}\right)$

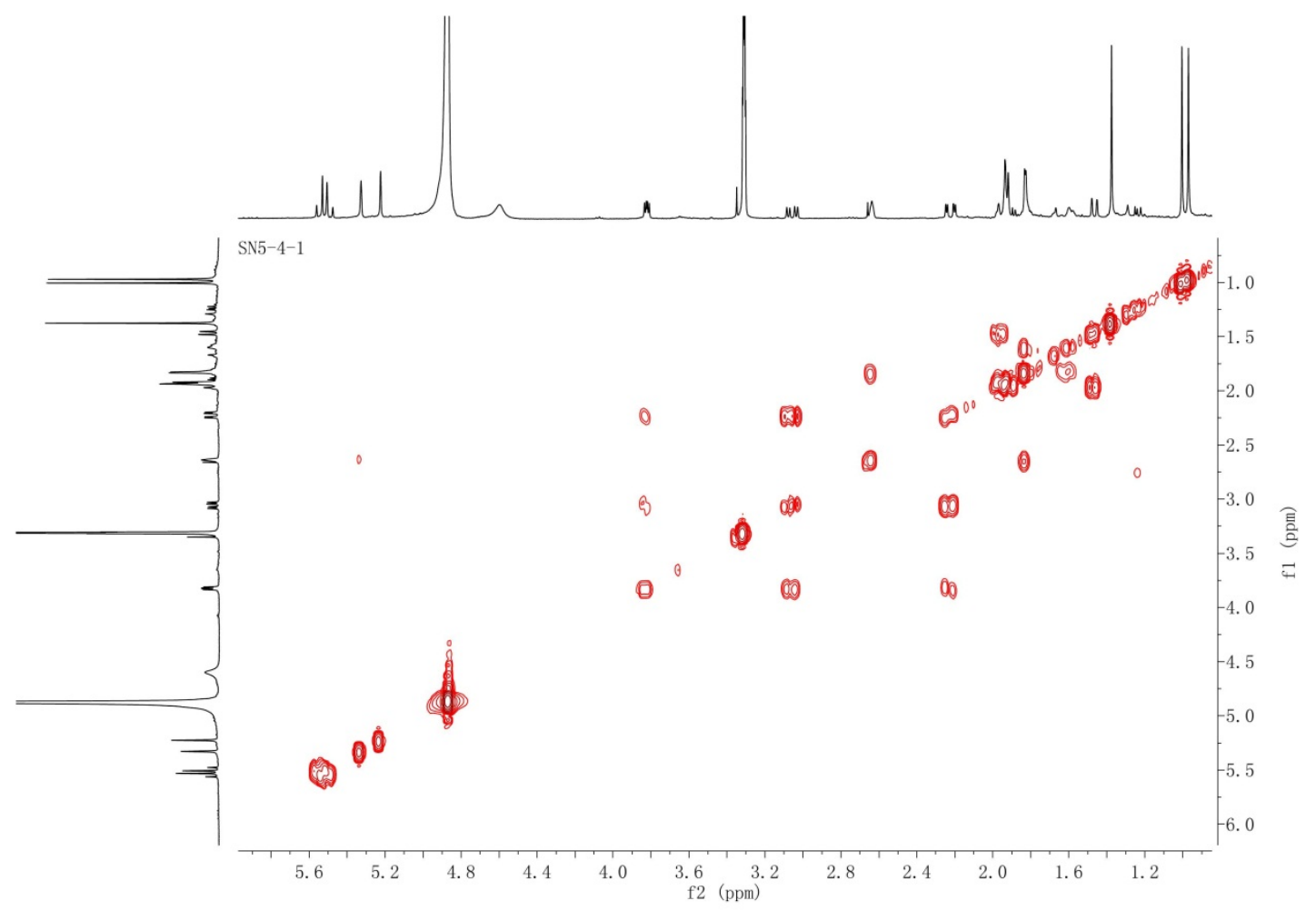

Figure S77. ${ }^{1} \mathrm{H}-{ }^{1} \mathrm{H}$ COSY (400 MHz, methanol-d4) spectrum of compound 8 


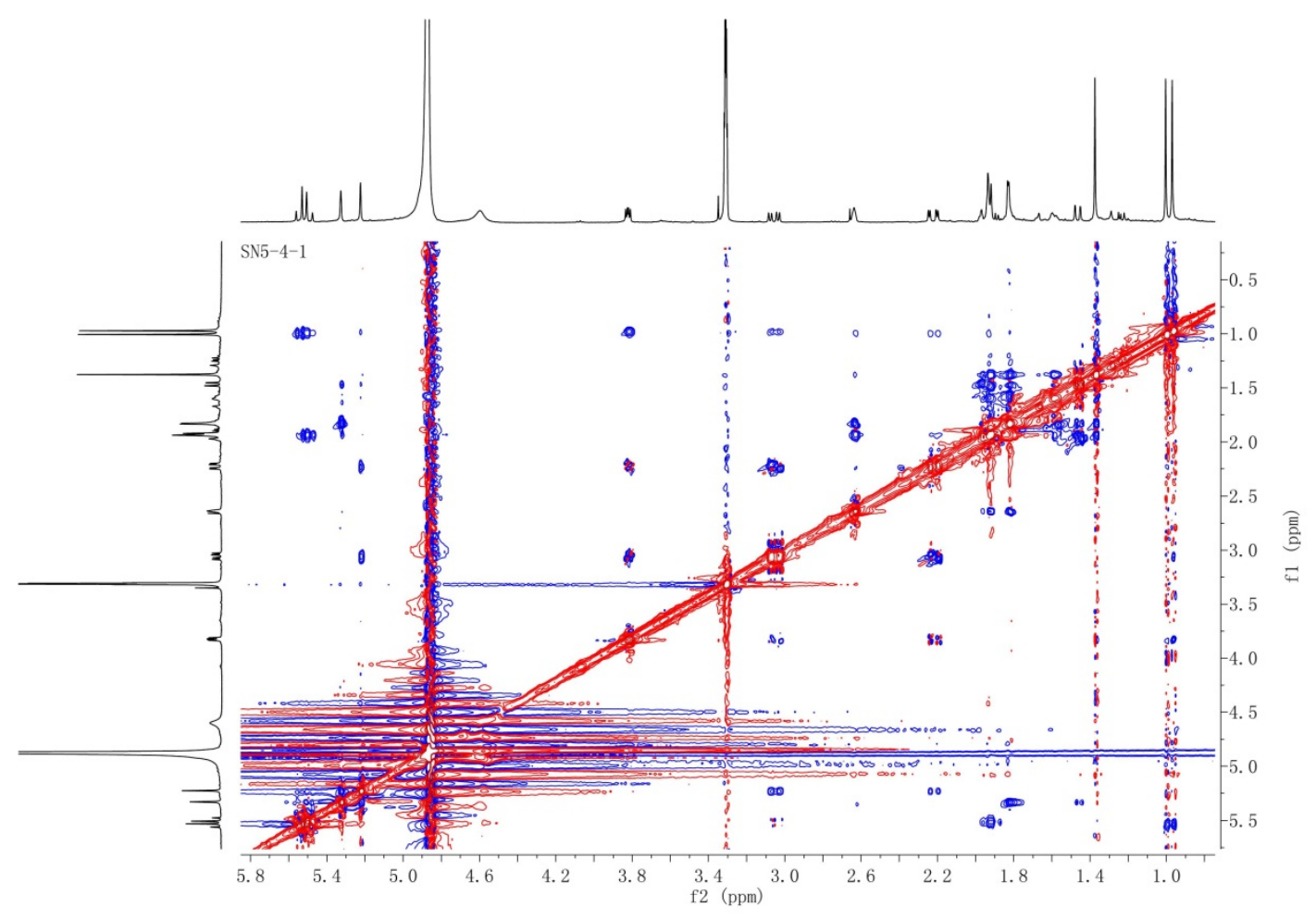

Figure S78. NOESY (400 MHz, methanol- $d_{4}$ ) spectrum of compound 8

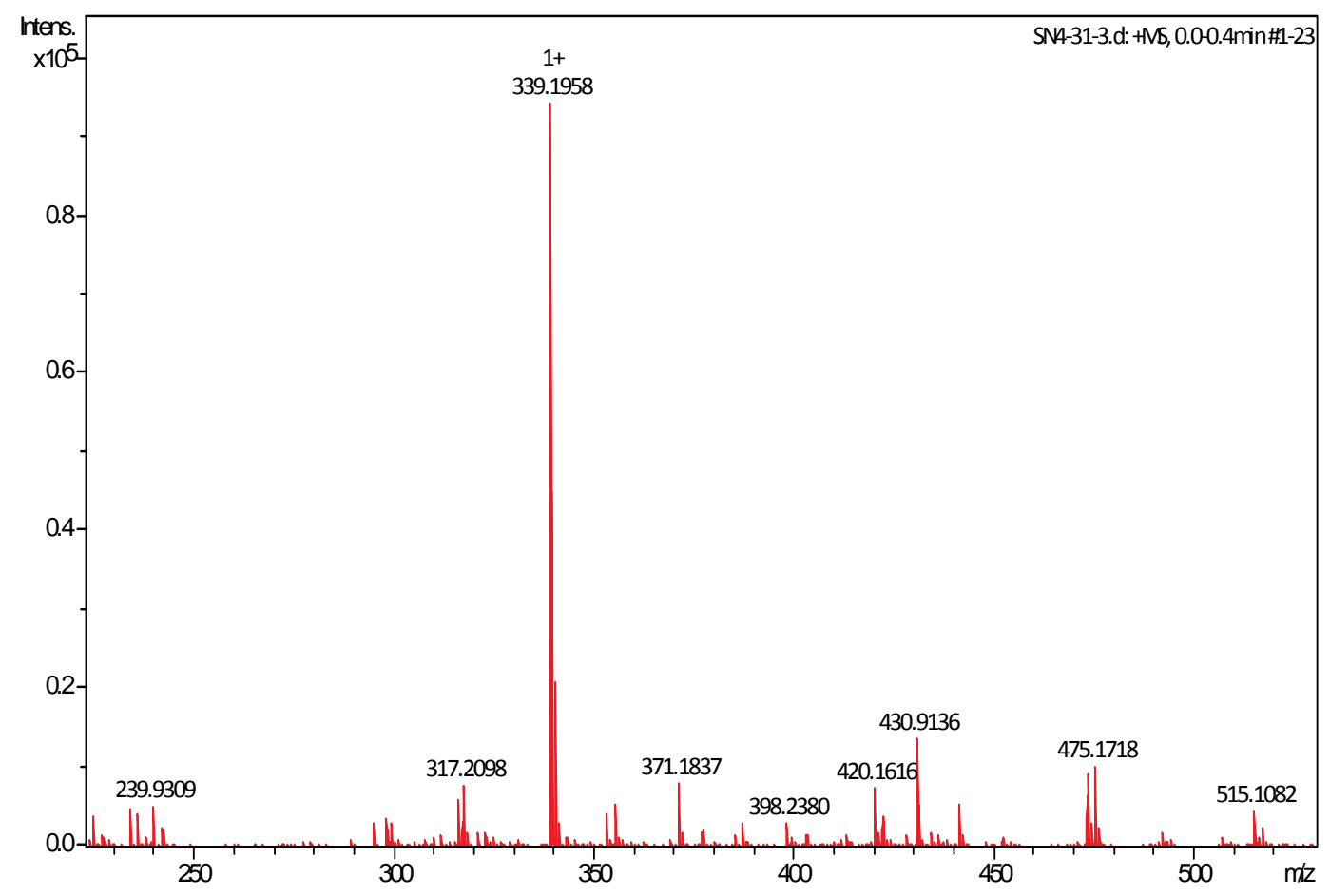

Figure S79. (+)-HRESIMS of compound 9 


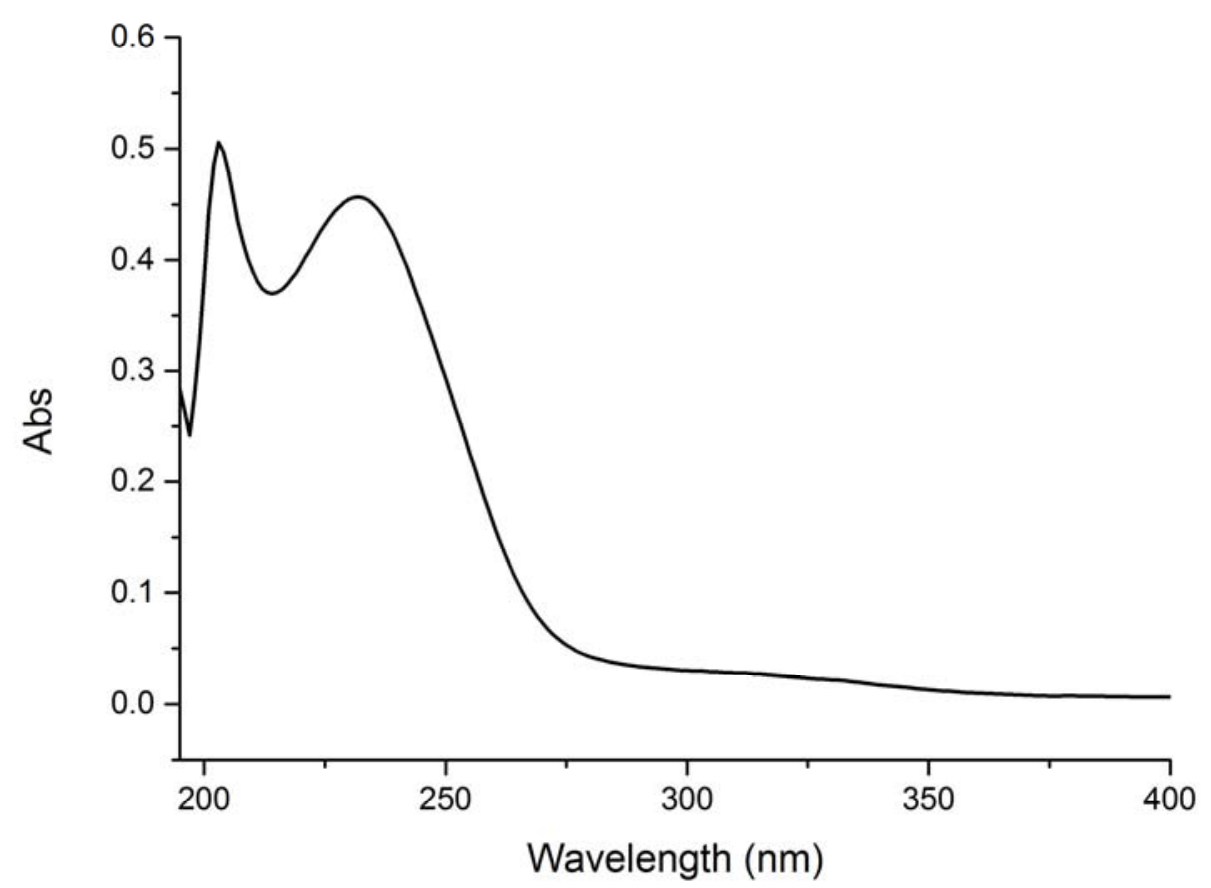

Figure S80. UV spectrum of compound 9 in $\mathrm{MeOH}$

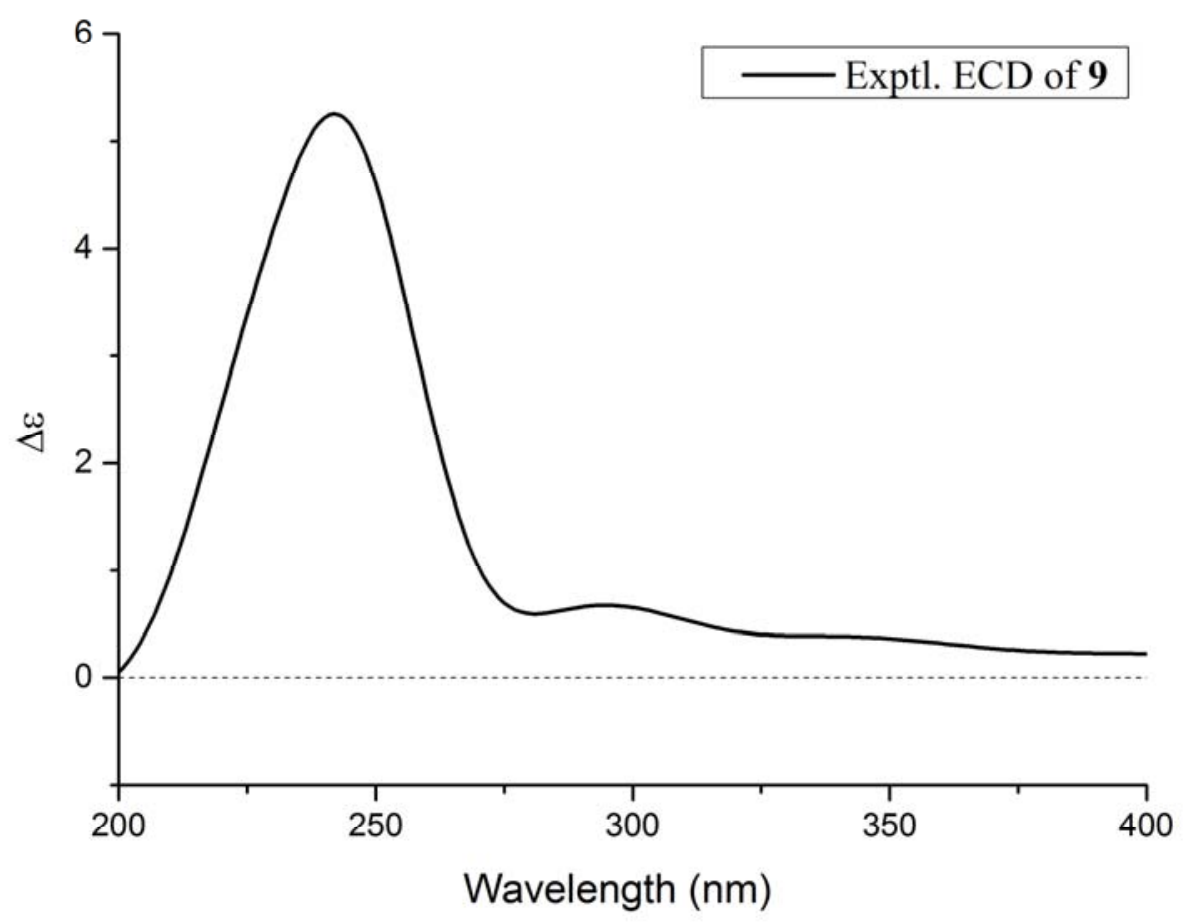

Figure S81. CD spectrum of compound 9 in $\mathrm{MeOH}$ 


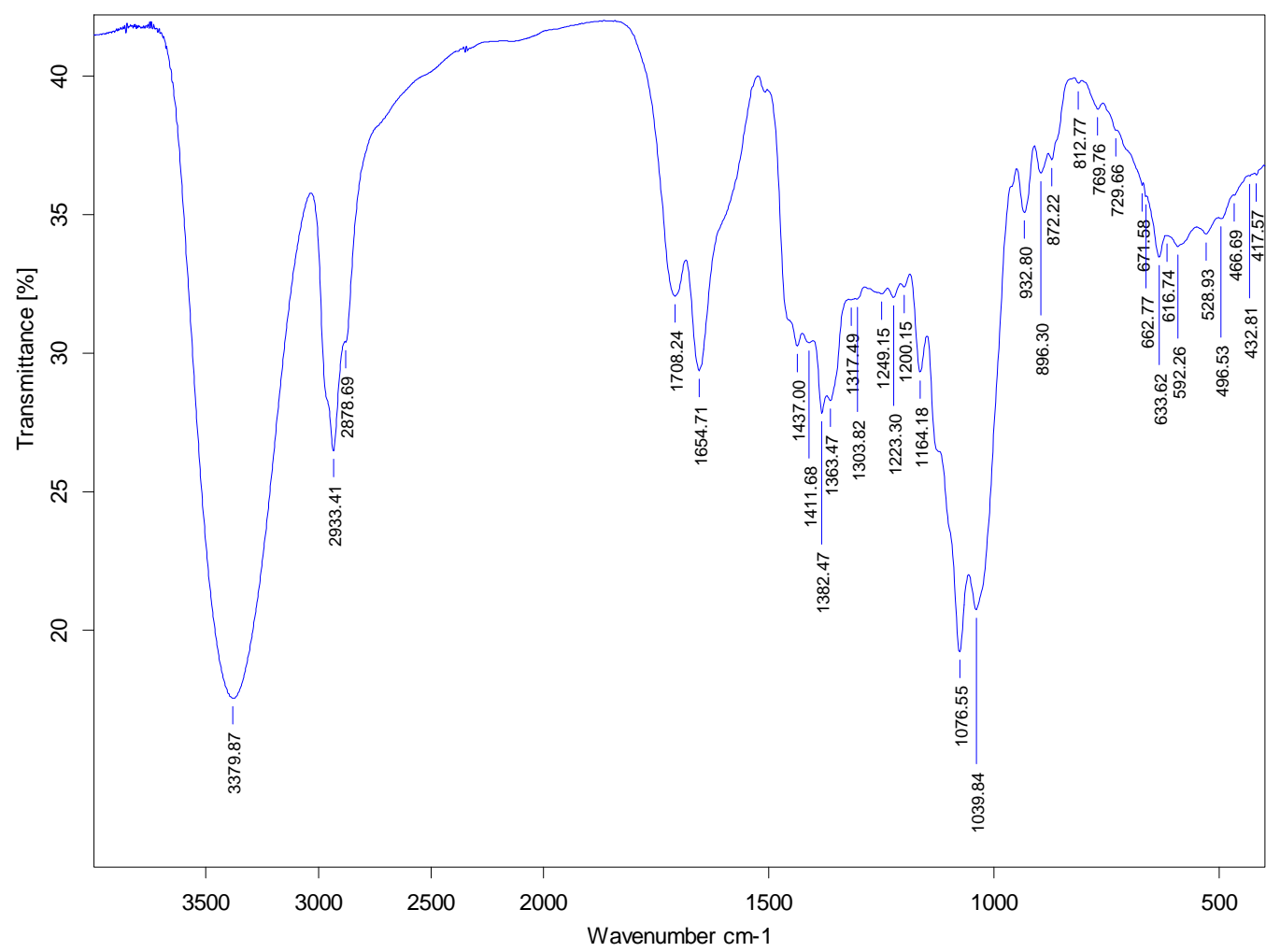

Figure S82. IR of compound 9

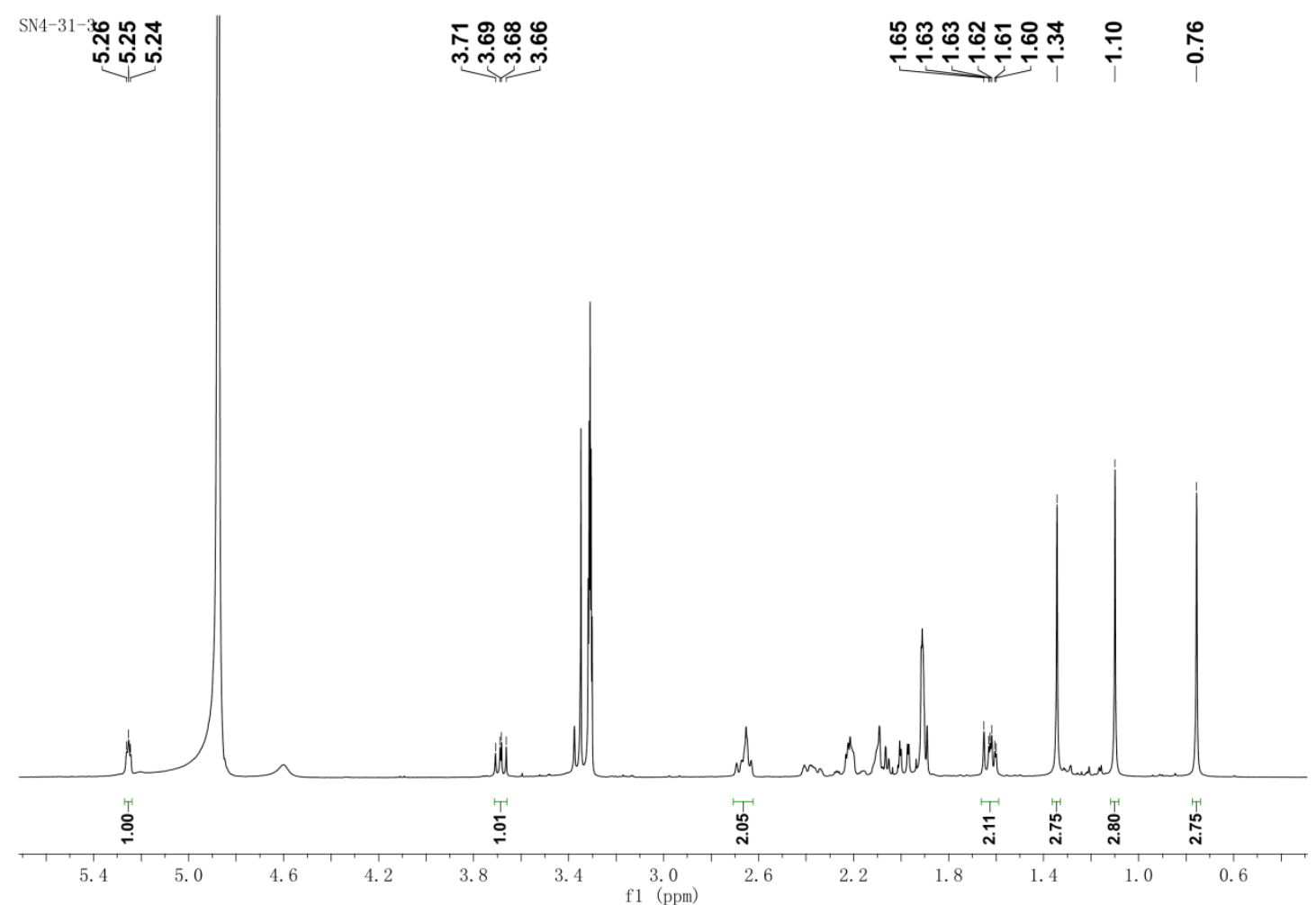

Figure S83. ${ }^{1} \mathrm{H}$ NMR (400 MHz, methanol- $d_{4}$ ) spectrum of compound 9 


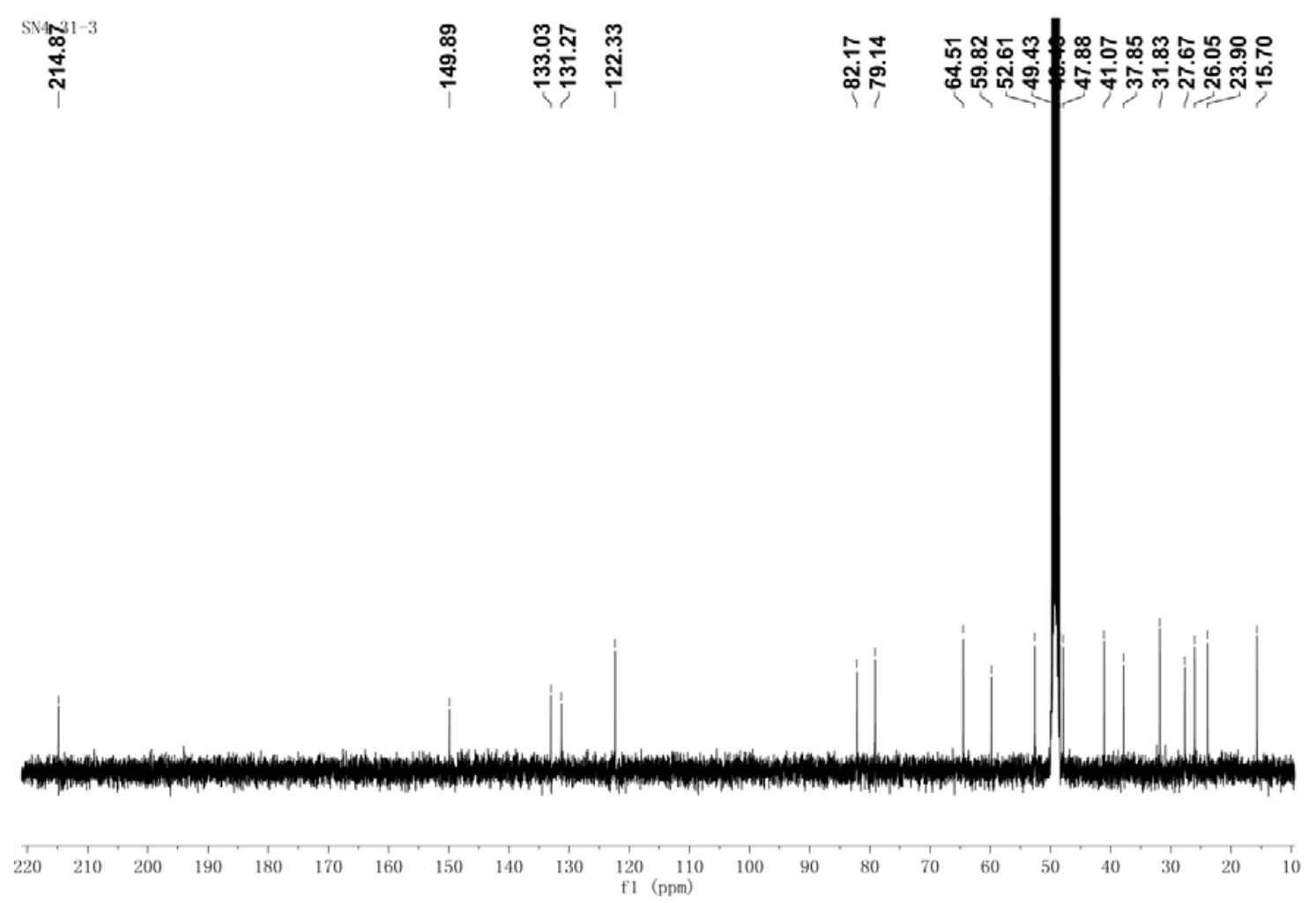

Figure S84. ${ }^{13} \mathrm{C}$ NMR (100 MHz, methanol- $\left.d_{4}\right)$ spectrum of compound 9
SN4-31- ָָ ָָ
$\stackrel{m}{\stackrel{m}{i}}$
号

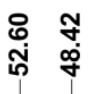

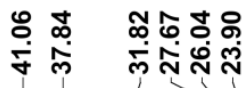
$\stackrel{8}{\stackrel{8}{\circ}}$

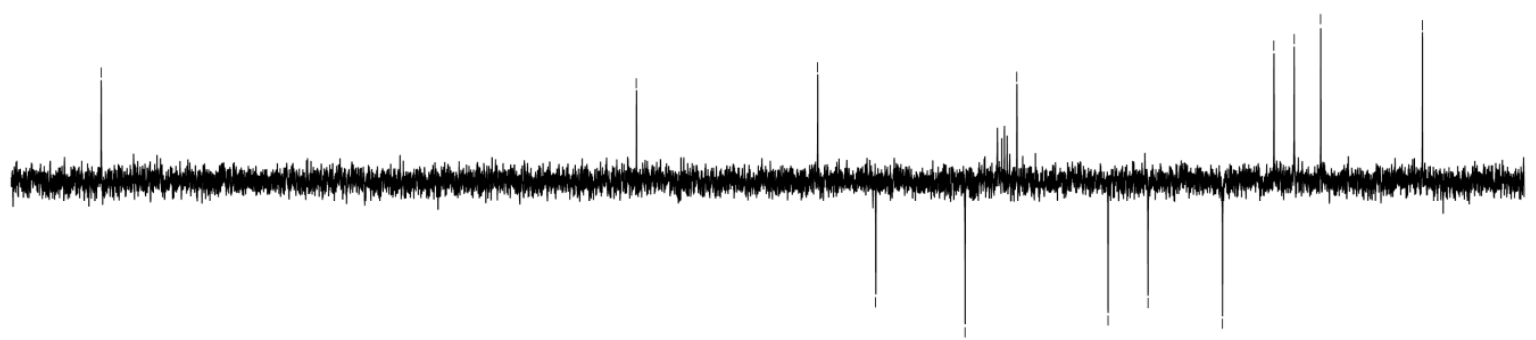

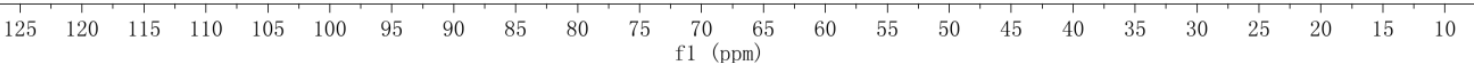

Figure S85. DEPT (100 MHz, methanol- $\left.d_{4}\right)$ spectrum of compound 9 


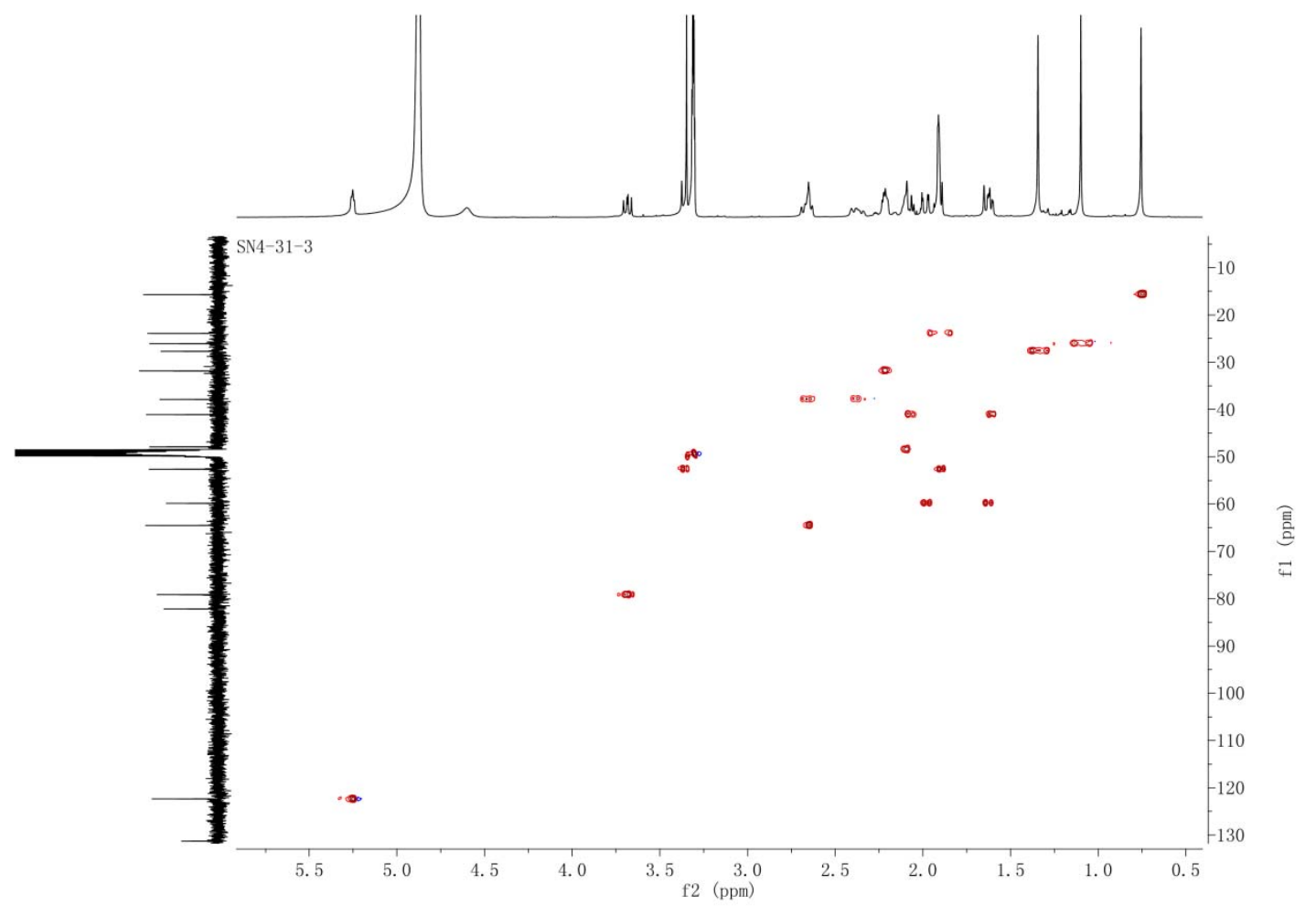

Figure S86. HSQC (methanol- $\left.d_{4}\right)$ spectrum of compound $9\left({ }^{1} \mathrm{H}: 400 \mathrm{MHz},{ }^{13} \mathrm{C}: 100 \mathrm{MHz}\right)$

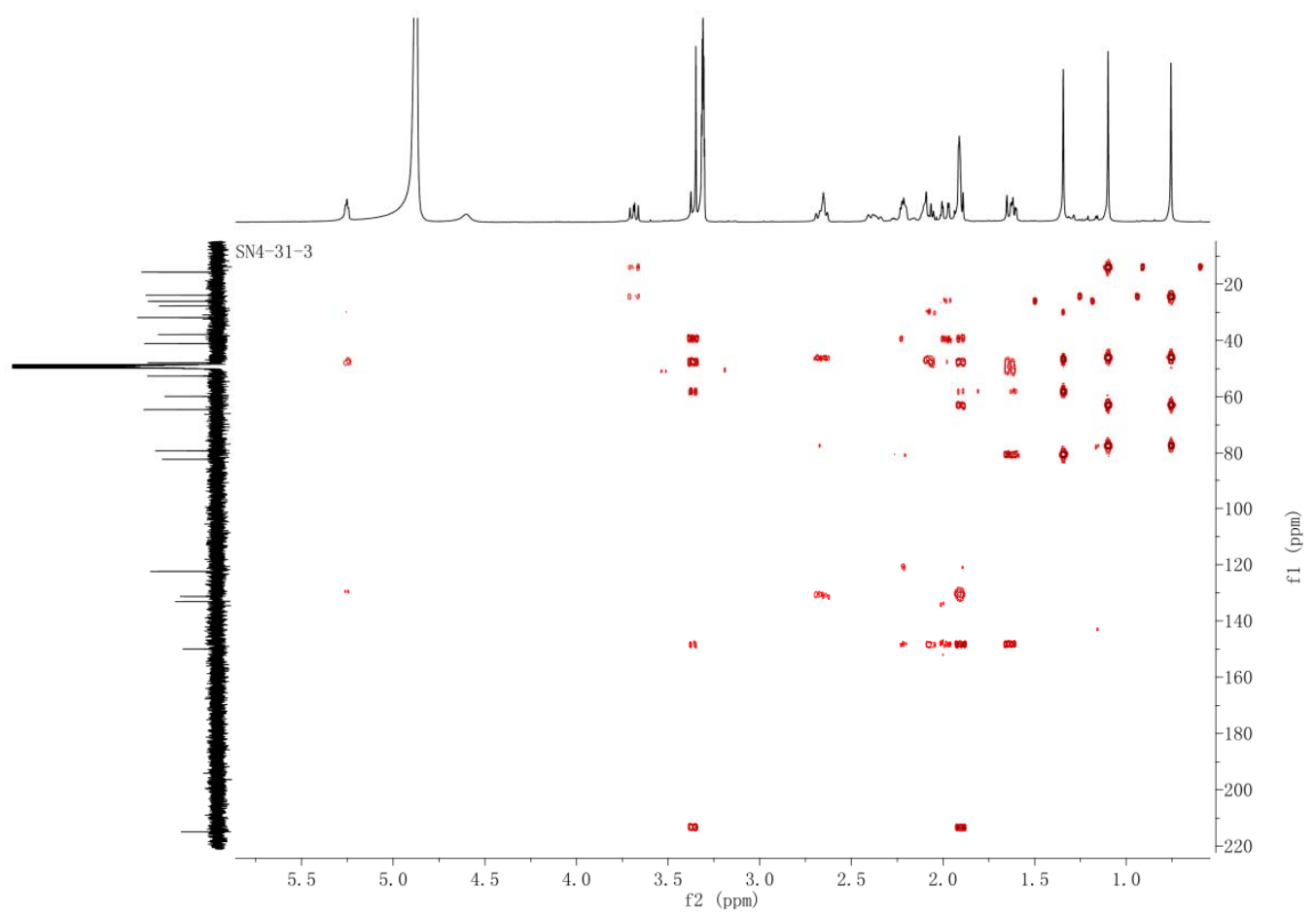

Figure S87. HMBC (methanol- $\left.d_{4}\right)$ spectrum of compound $9\left({ }^{1} \mathrm{H}: 400 \mathrm{MHz},{ }^{13} \mathrm{C}: 100 \mathrm{MHz}\right)$ 


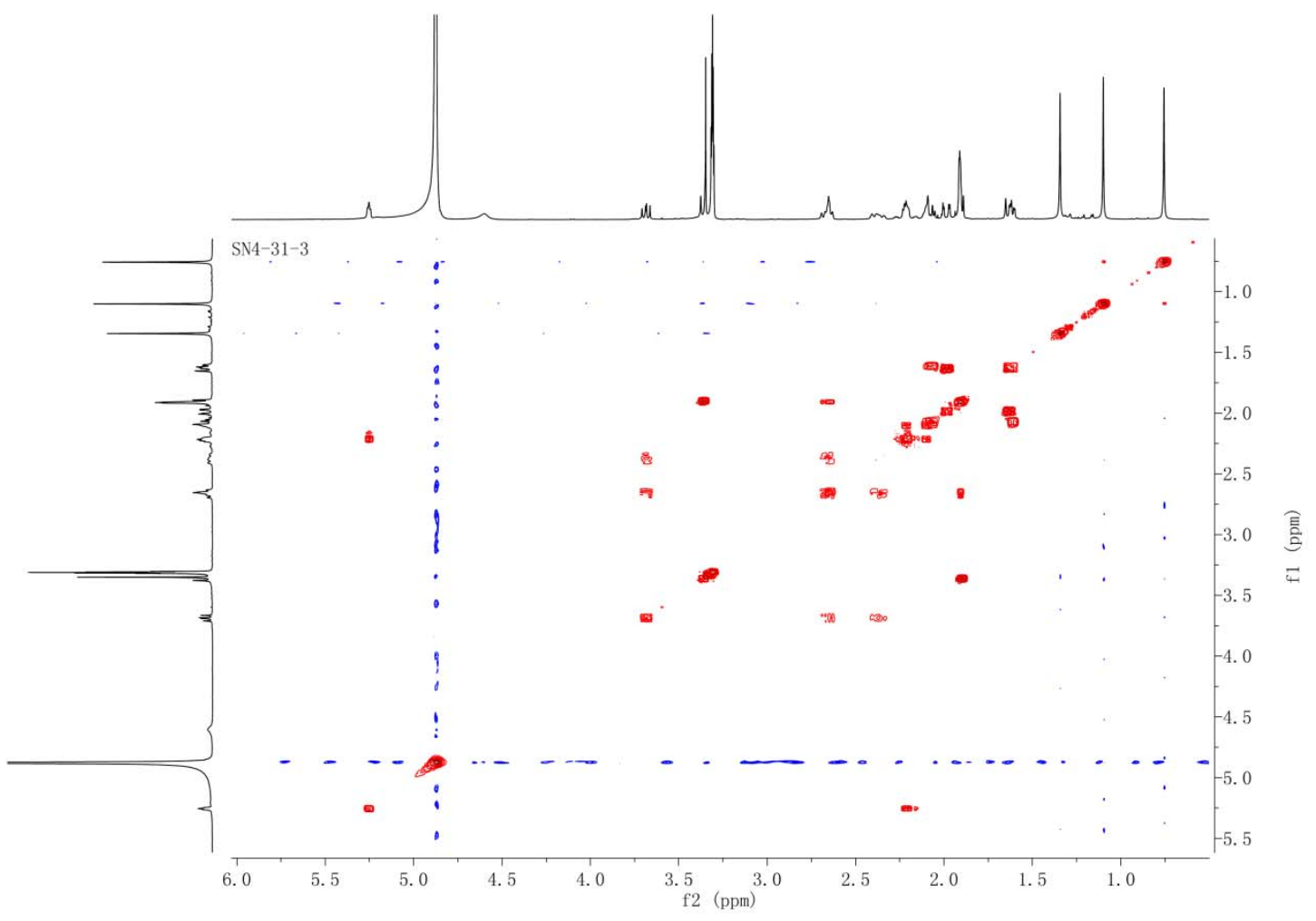

Figure S88. The ${ }^{1} \mathrm{H}^{-1} \mathrm{H}$ COSY $\left(400 \mathrm{MHz}\right.$, methanol- $\left.d_{4}\right)$ spectrum of compound 9

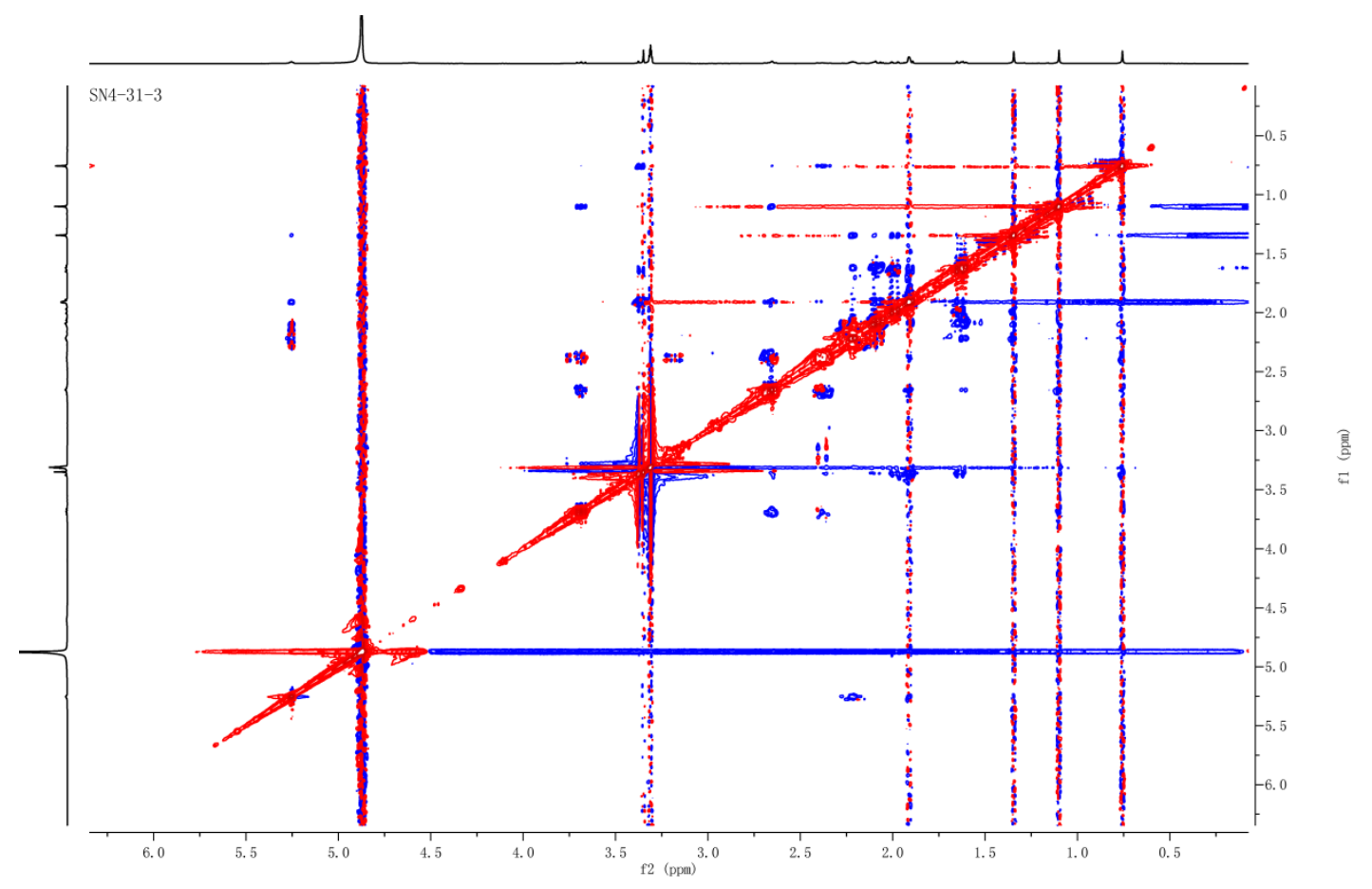

Figure S89. The NOESY ( $400 \mathrm{MHz}$, methanol- $d_{4}$ ) spectrum of compound 9 


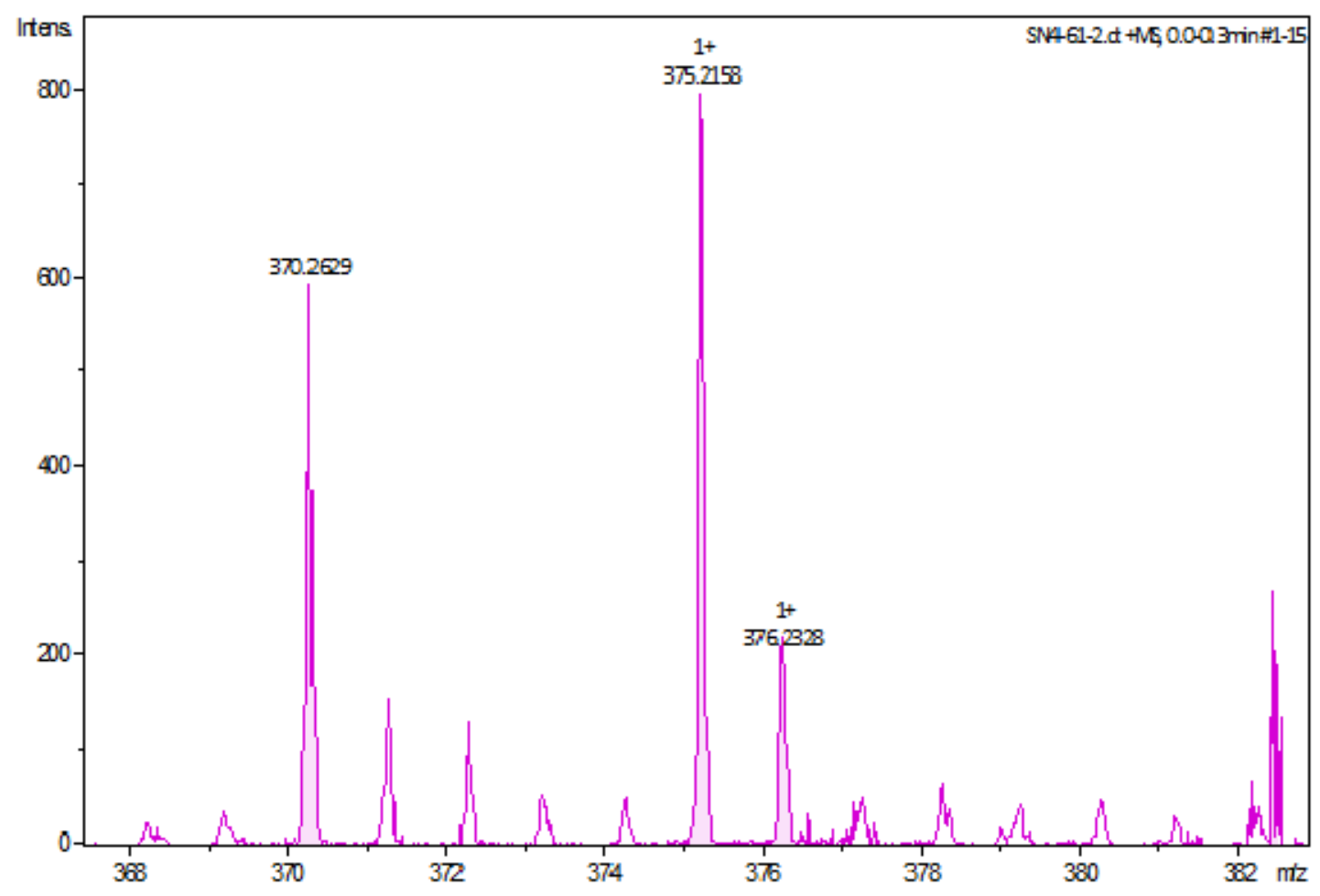

Figure S90. (+)-HRESIMS of compound 10

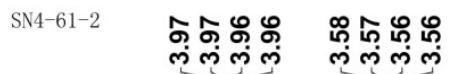

$\sin 5 \sin$

iving

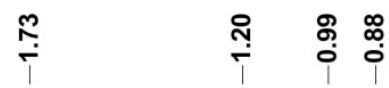

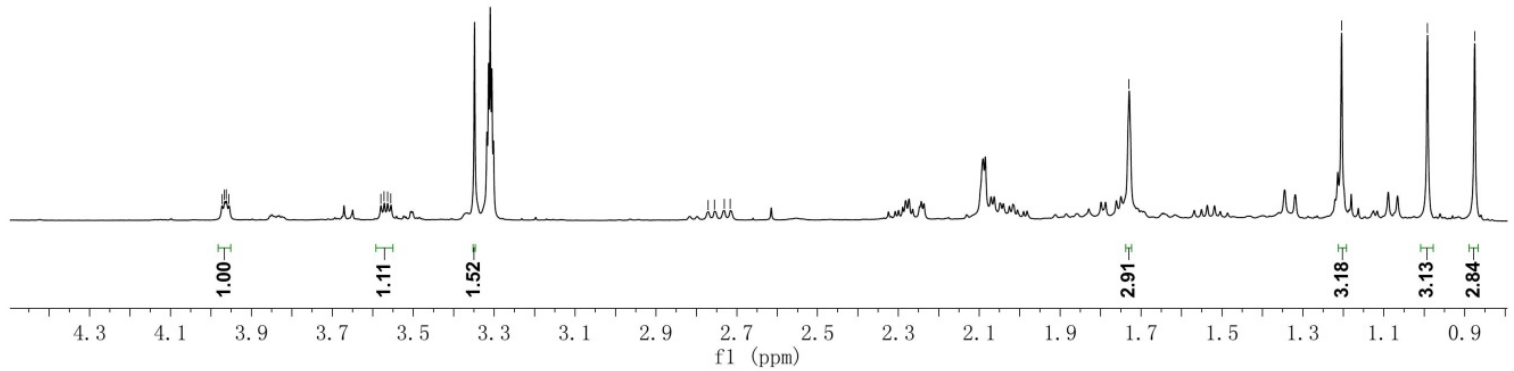

Figure S91. ${ }^{1} \mathrm{H}$ NMR (400 MHz, methanol- $\left.d_{4}\right)$ spectrum of compound 10 


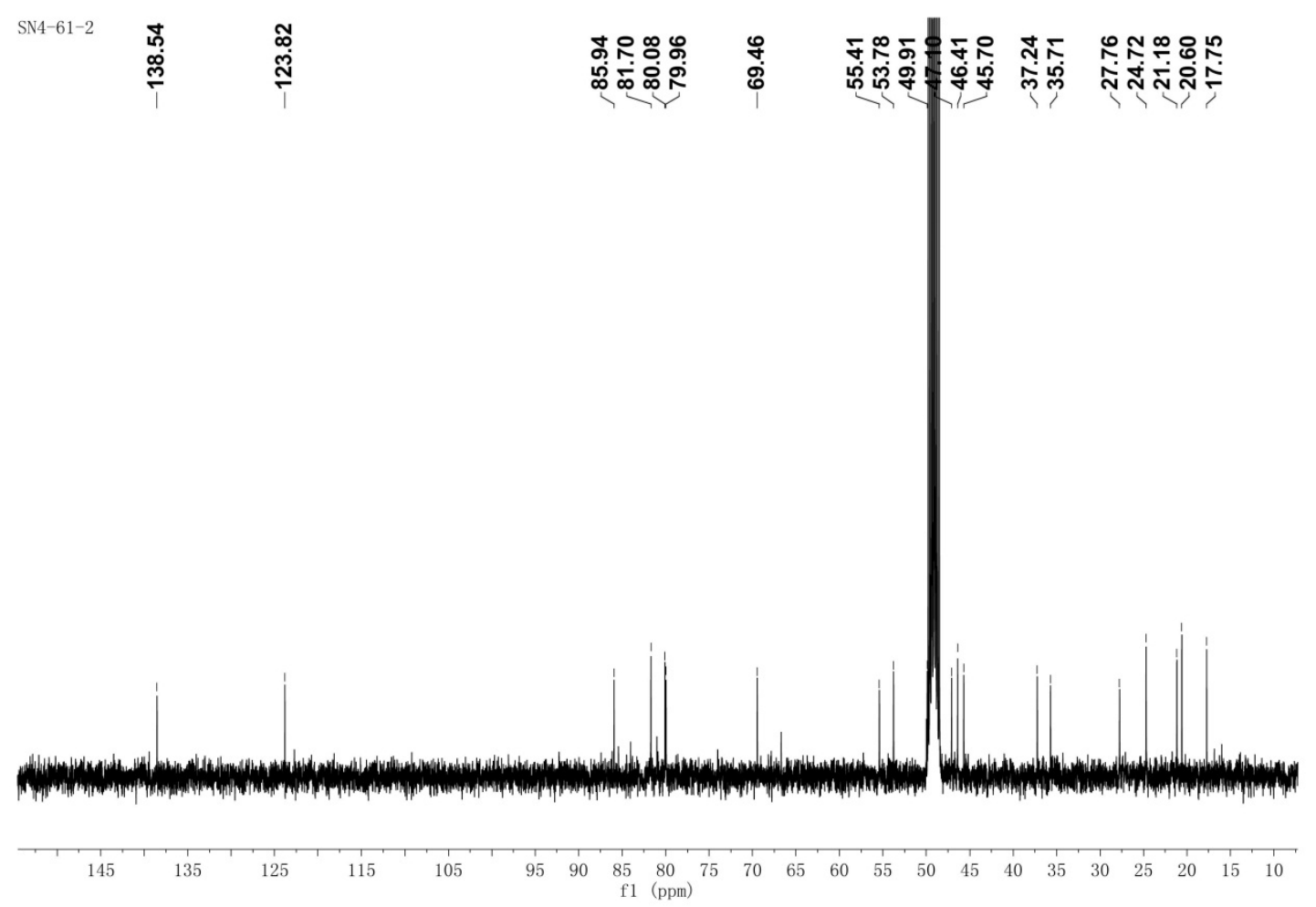

Figure S92. ${ }^{13} \mathrm{C}$ NMR (100 MHz, methanol- $\left.d_{4}\right)$ spectrum of compound 10

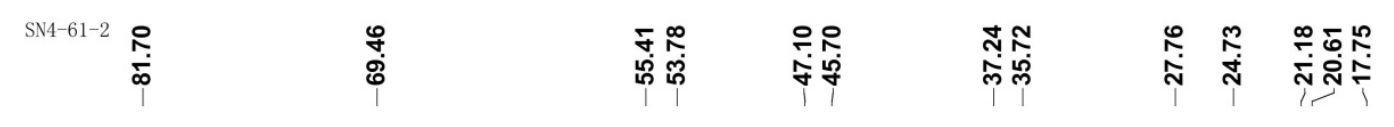

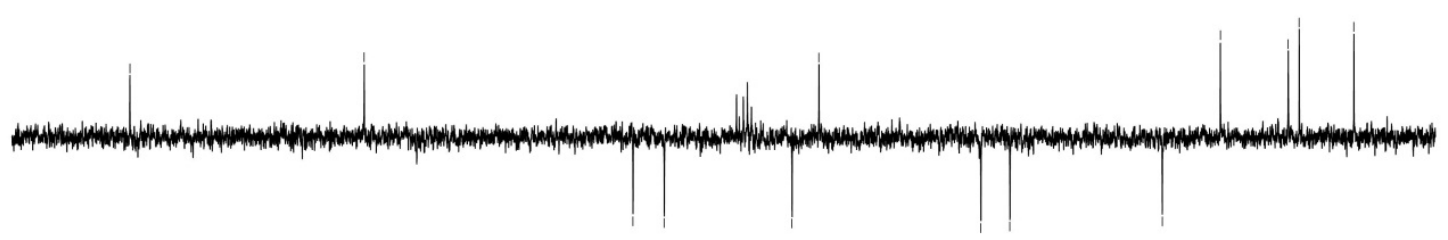

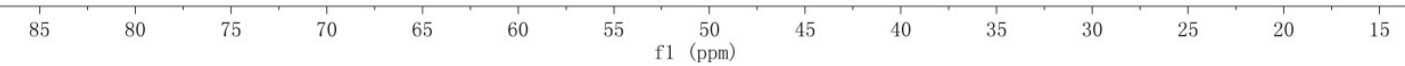

Figure S93. DEPT (100 MHz, methanol- $\left.d_{4}\right)$ spectrum of compound 10 


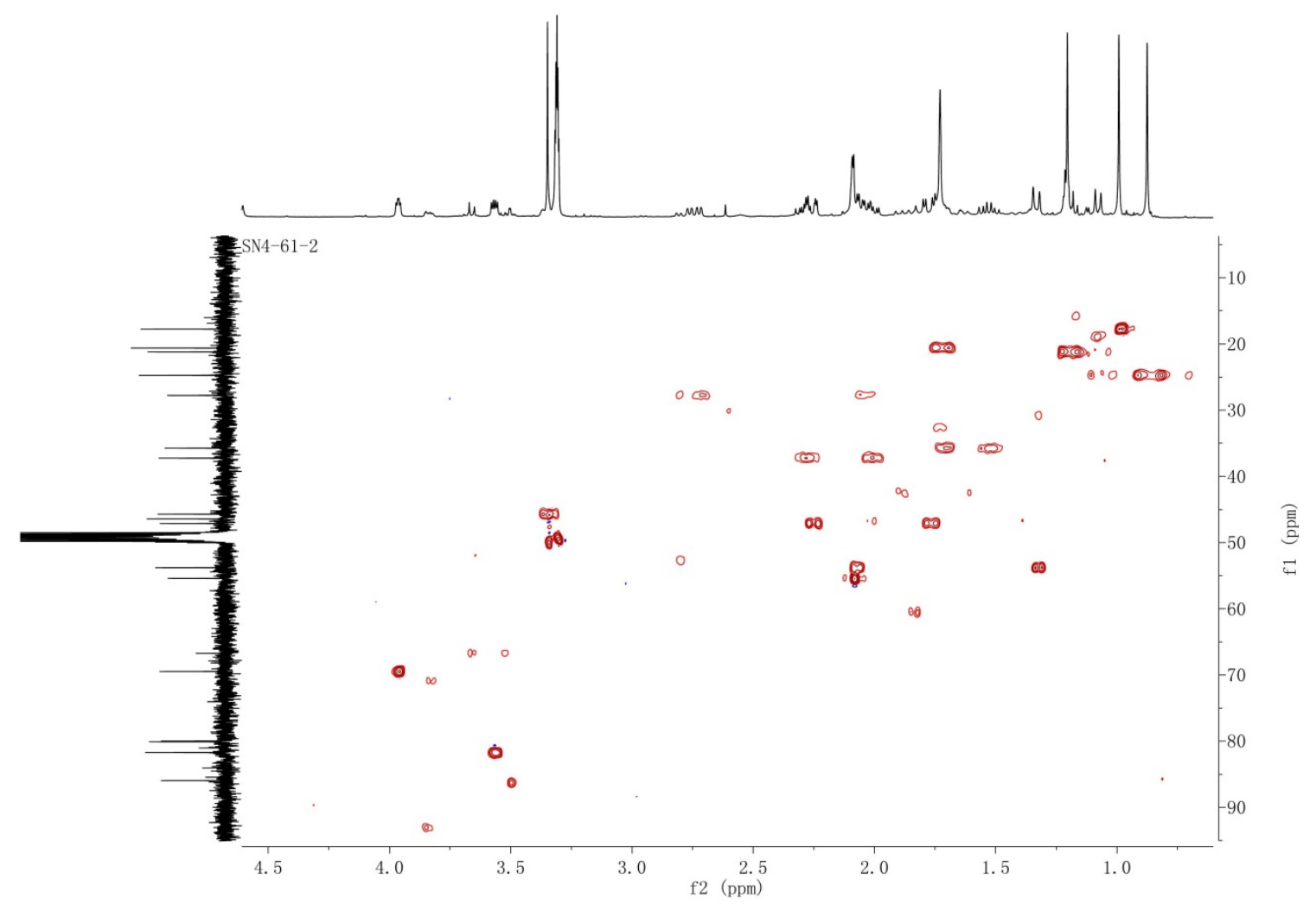

Figure S94. HSQC (methanol- $\left.d_{4}\right)$ spectrum of compound $10\left({ }^{1} \mathrm{H}: 400 \mathrm{MHz},{ }^{13} \mathrm{C}: 100 \mathrm{MHz}\right)$

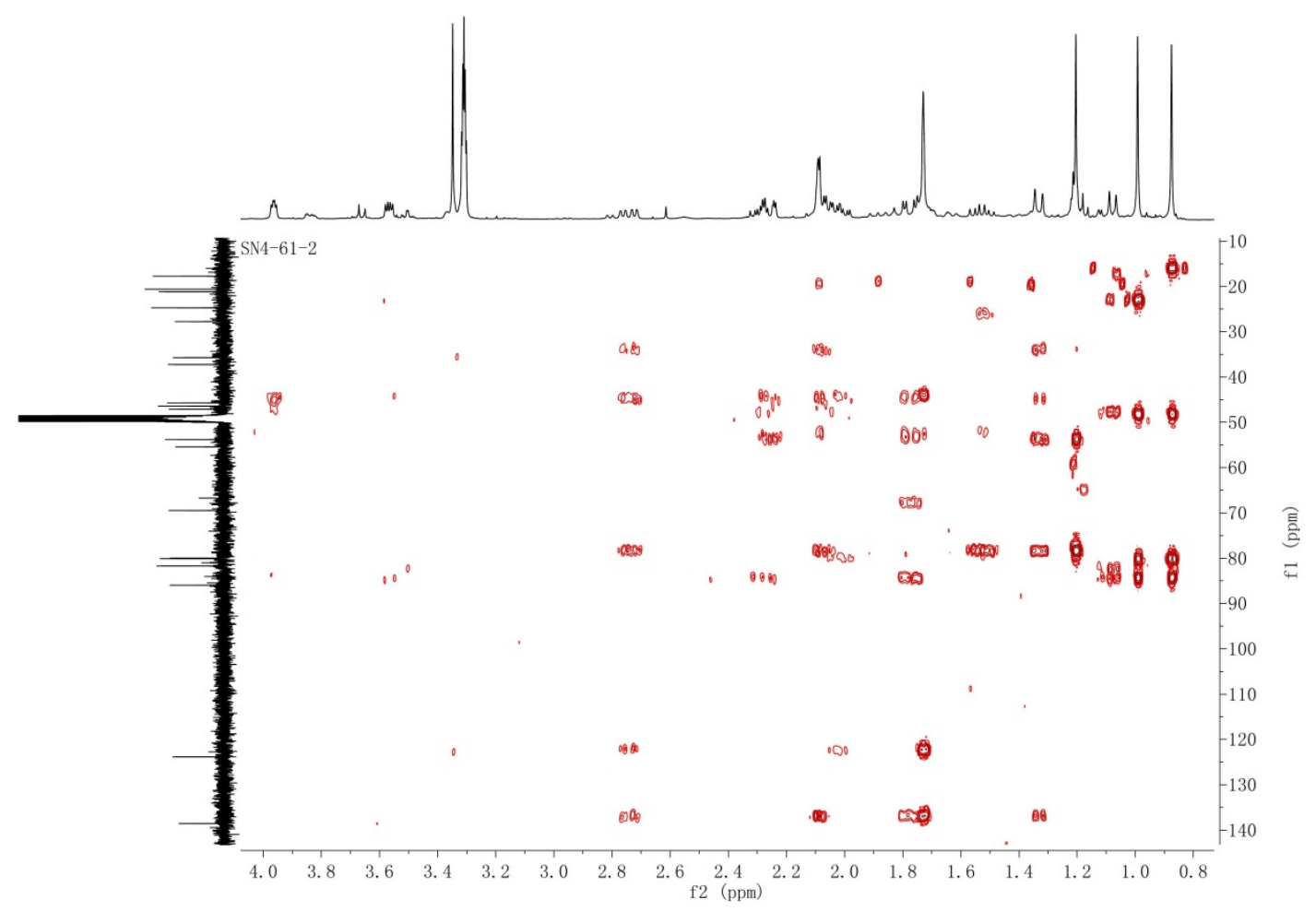

Figure S95. HMBC (methanol- $\left.d_{4}\right)$ spectrum of compound $10\left({ }^{1} \mathrm{H}: 400 \mathrm{MHz},{ }^{13} \mathrm{C}: 100 \mathrm{MHz}\right)$ 


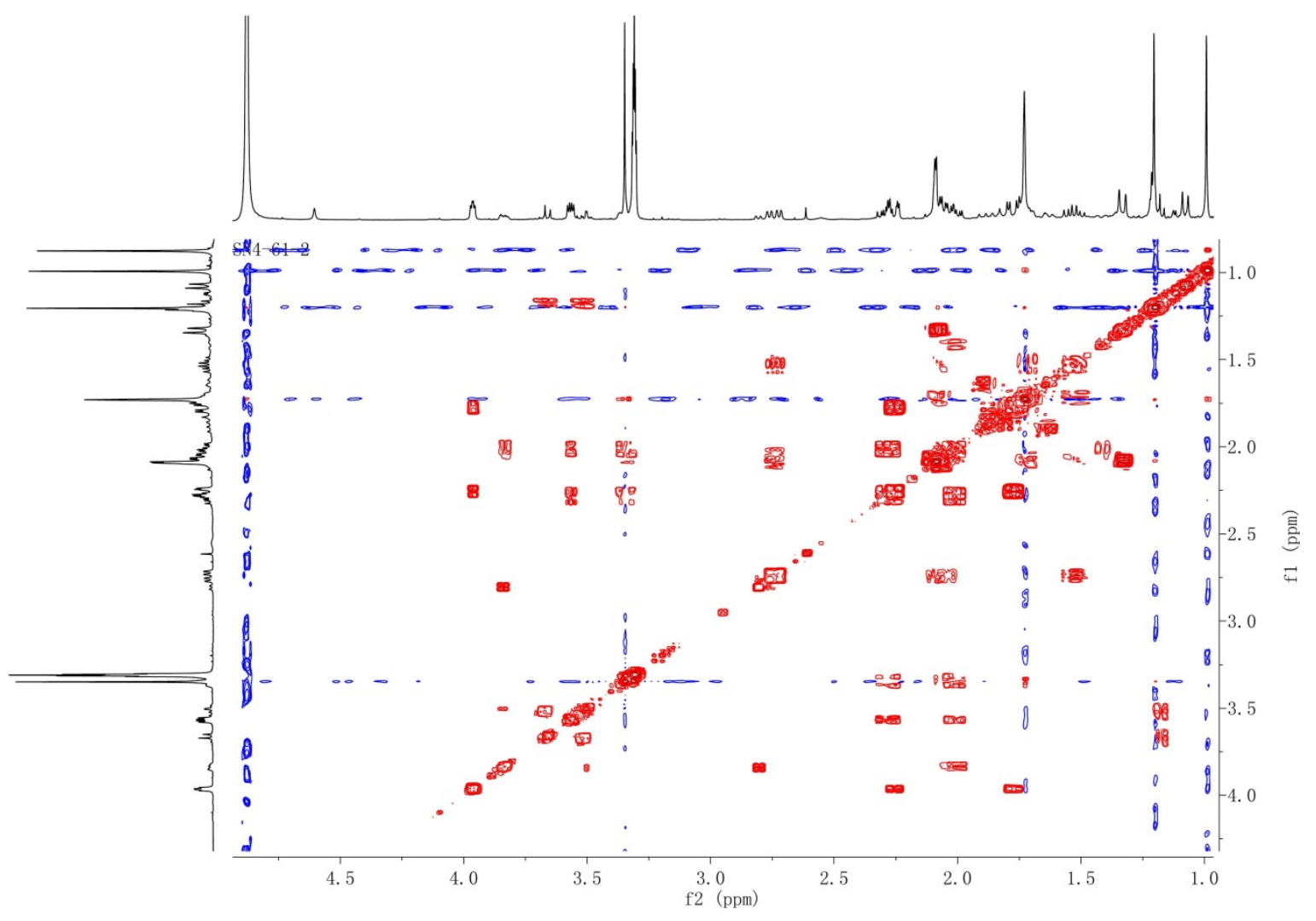

Figure S96. The ${ }^{1} \mathrm{H}-{ }^{1} \mathrm{H}$ COSY (400 MHz, methanol- $d_{4}$ ) spectrum of compound 10

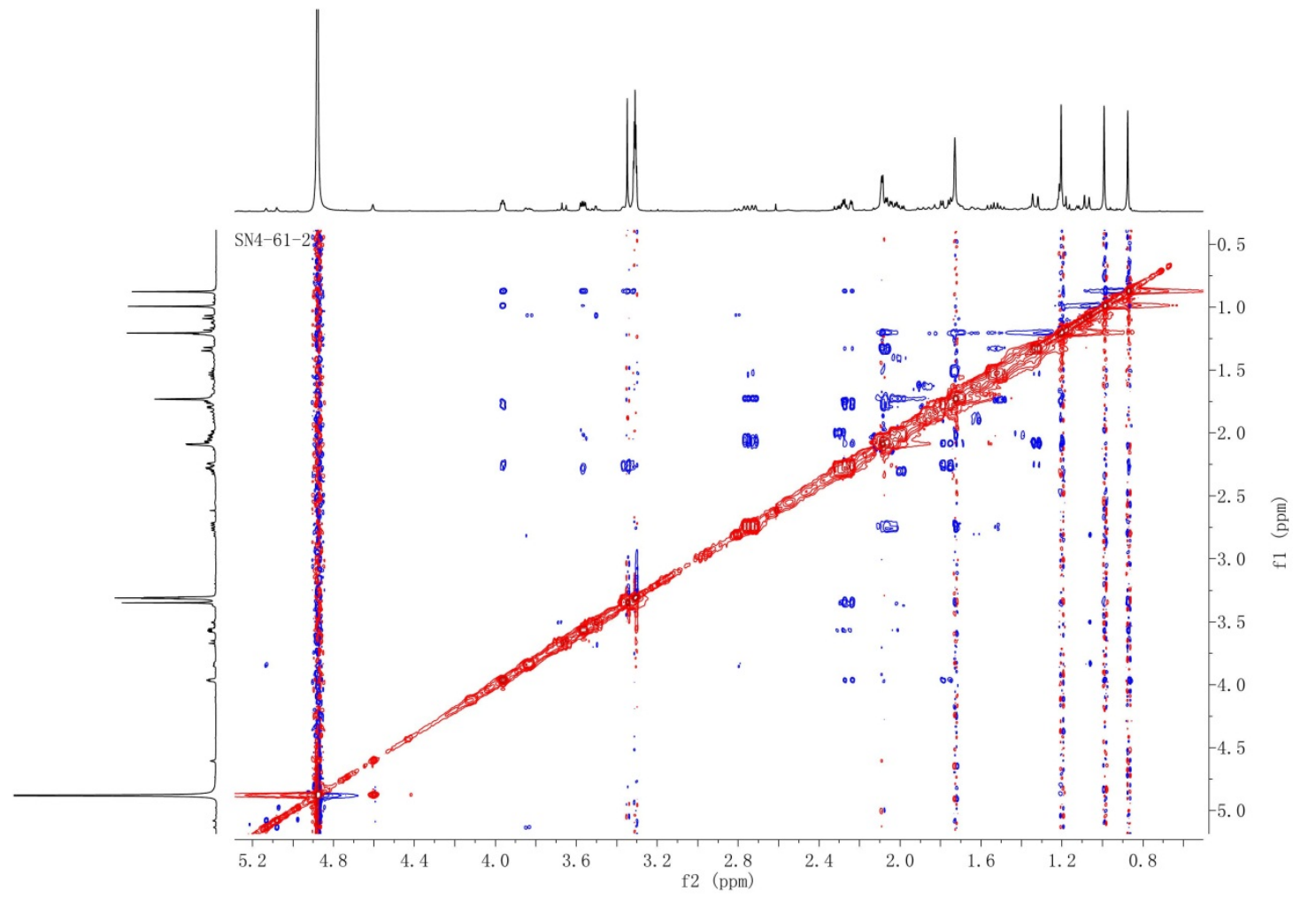

Figure S97. The NOESY (400 MHz, methanol- $\left.d_{4}\right)$ spectrum of compound 10 


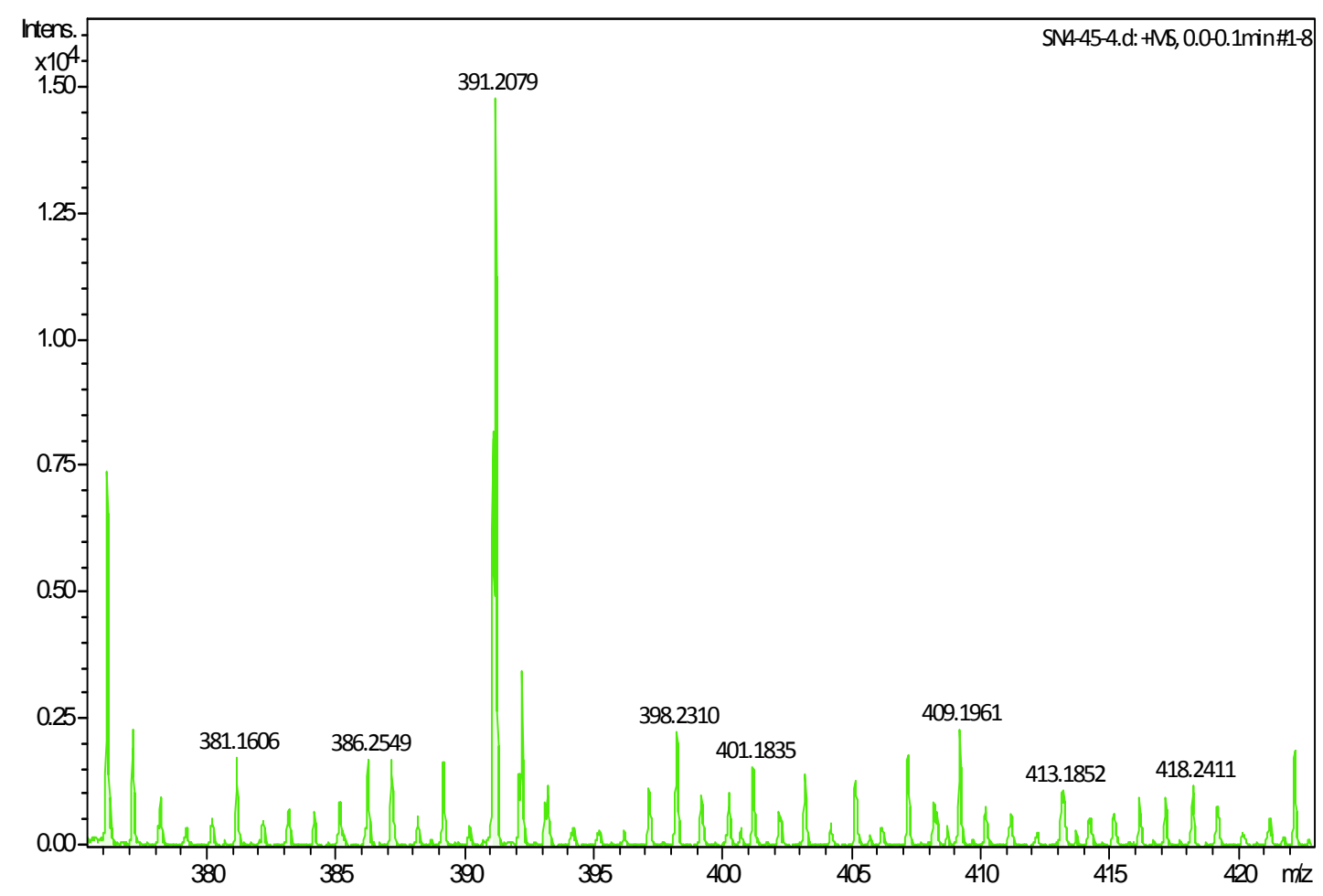

Figure S98. (+)-HRESIMS of compound 11

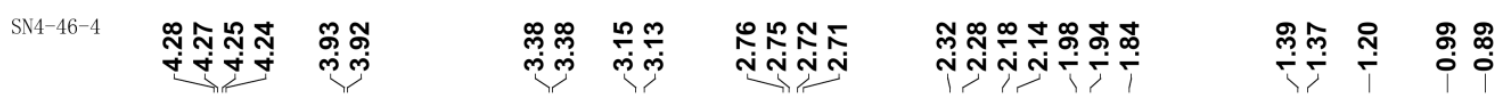

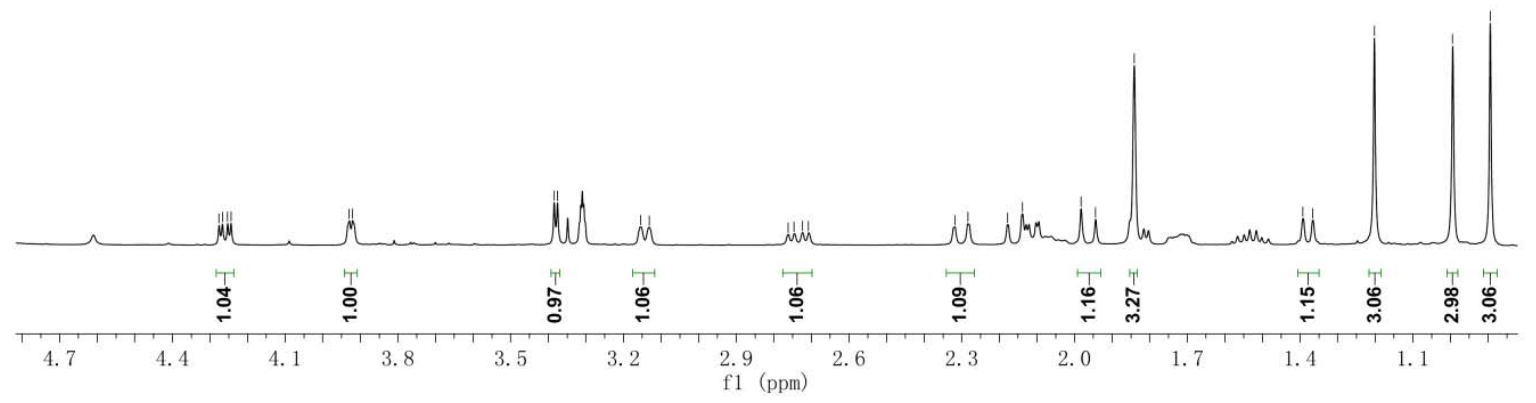

Figure S99. ${ }^{1} \mathrm{H}$ NMR (400 MHz, methanol-d $)$ spectrum of compound 11 


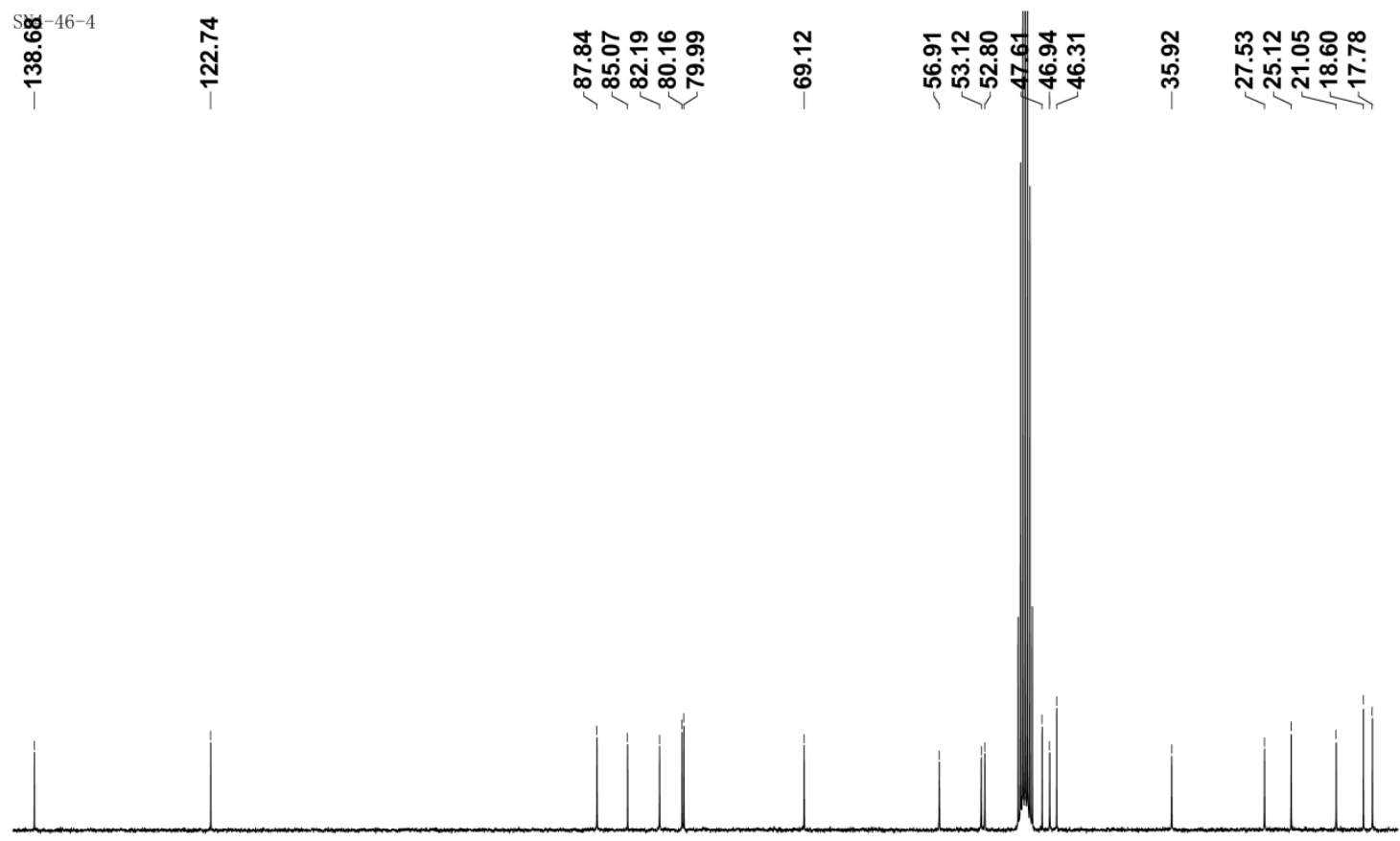

$\begin{array}{lllllllllllllllllllllllllllllllll} & 130 & 135 & 130 & 125 & 120 & 115 & 110 & 105 & 100 & 95 & 90 & 85 & 80 & 75 & 70 & 65 & 60 & 55 & 50 & 45 & 40 & 35 & 30 & 25 & 20\end{array}$

Figure S100. ${ }^{13} \mathrm{C}$ NMR (100 MHz, methanol- $\left.d_{4}\right)$ spectrum of compound 11

SN4-46-4

\begin{tabular}{|c|c|c|c|c|c|}
\hline$\underset{\infty}{\stackrel{\infty}{\leftarrow ்}}$ & ํ. & 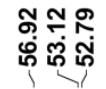 & 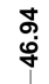 & \%ొ & 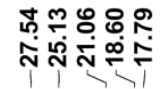 \\
\hline
\end{tabular}

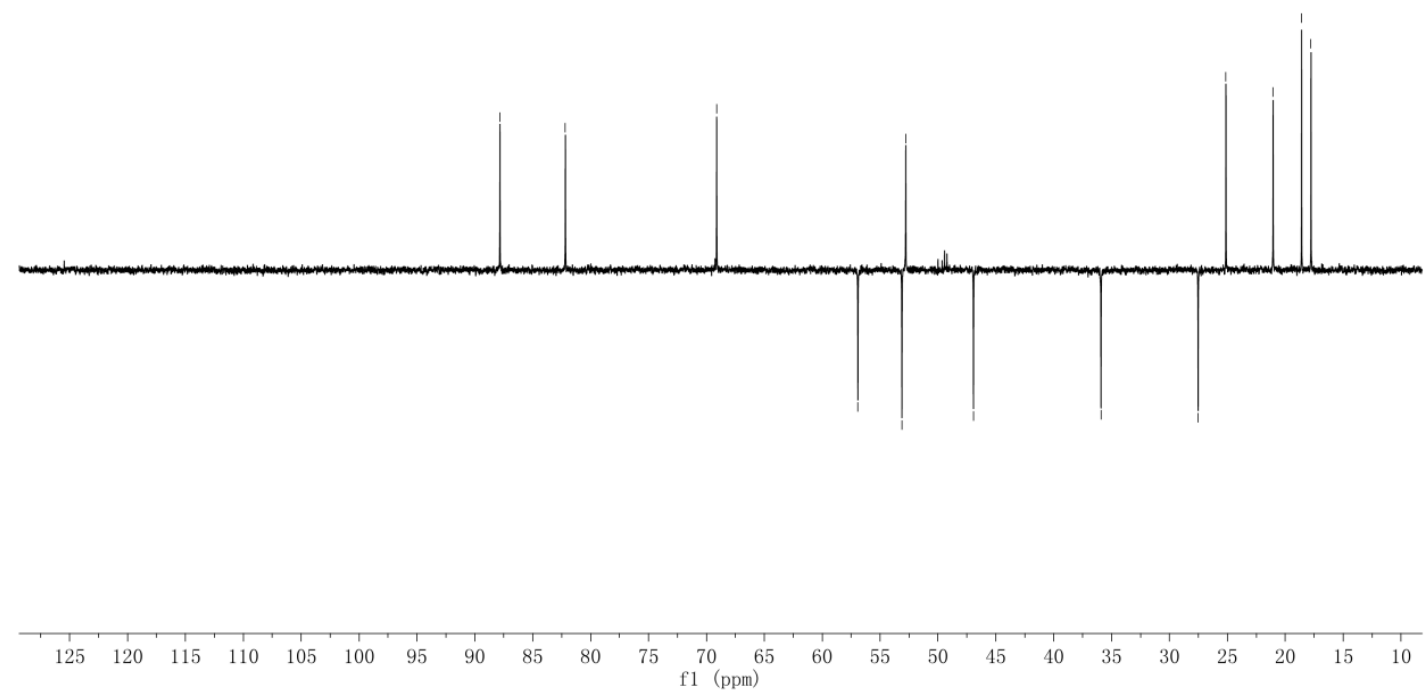

Figure S101. DEPT (100 MHz, methanol- $\left.d_{4}\right)$ spectrum of compound 11 


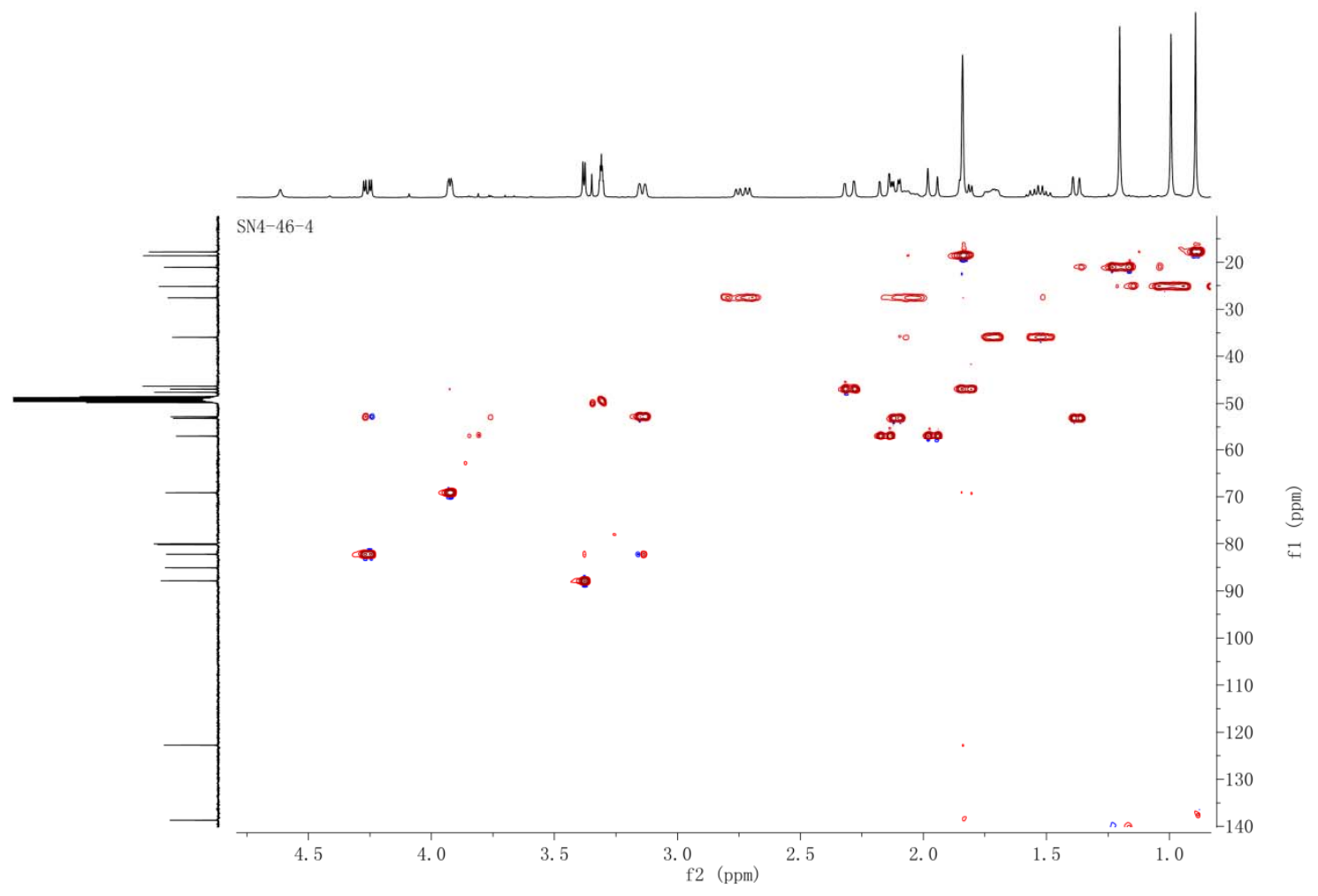

Figure S102. HSQC (methanol- $\left.d_{4}\right)$ spectrum of compound $11\left({ }^{1} \mathrm{H}: 400 \mathrm{MHz},{ }^{13} \mathrm{C}: 100 \mathrm{MHz}\right)$

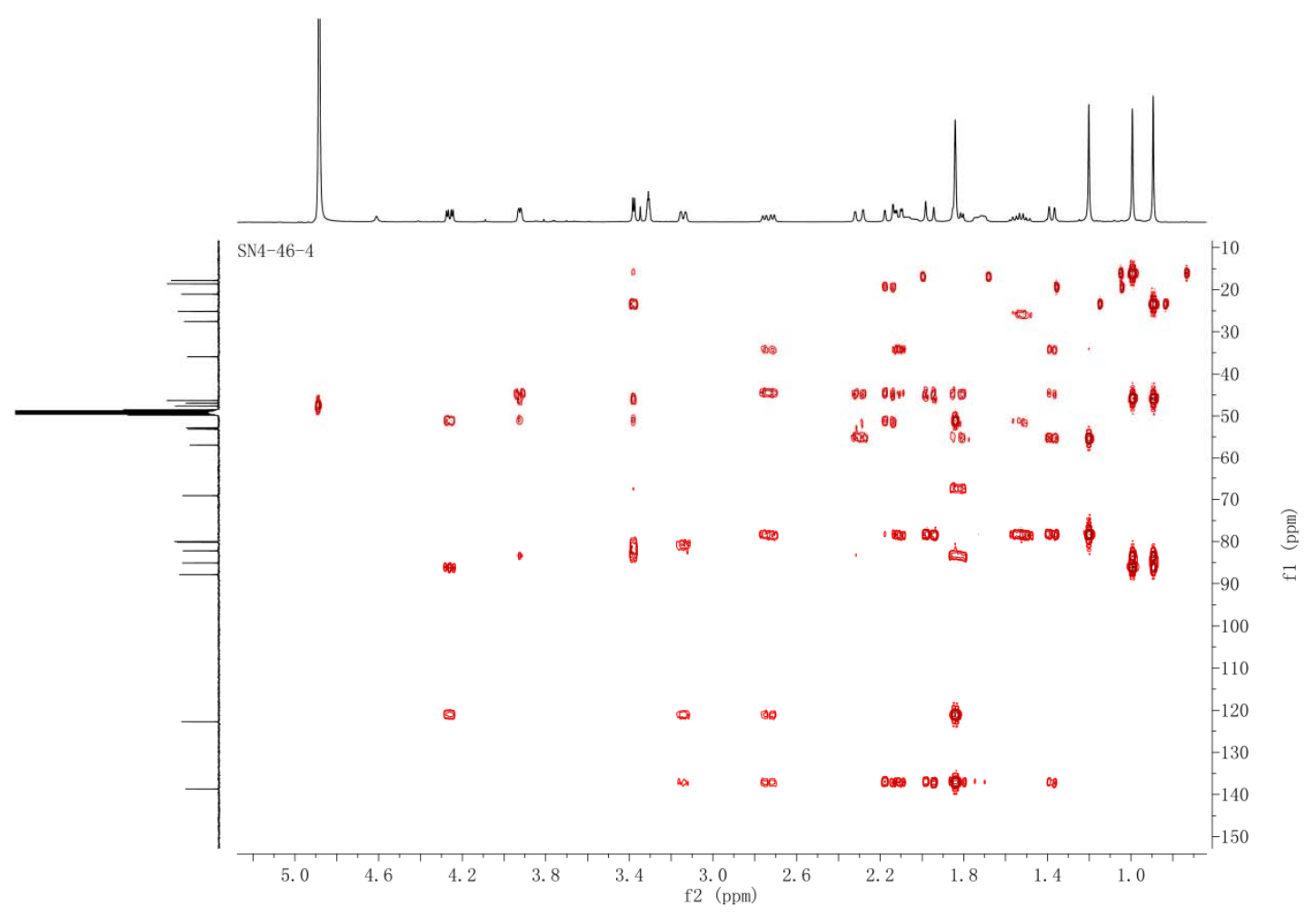

Figure S103. HMBC (methanol- $\left.d_{4}\right)$ spectrum of compound $11\left({ }^{1} \mathrm{H}: 400 \mathrm{MHz},{ }^{13} \mathrm{C}: 100 \mathrm{MHz}\right)$ 


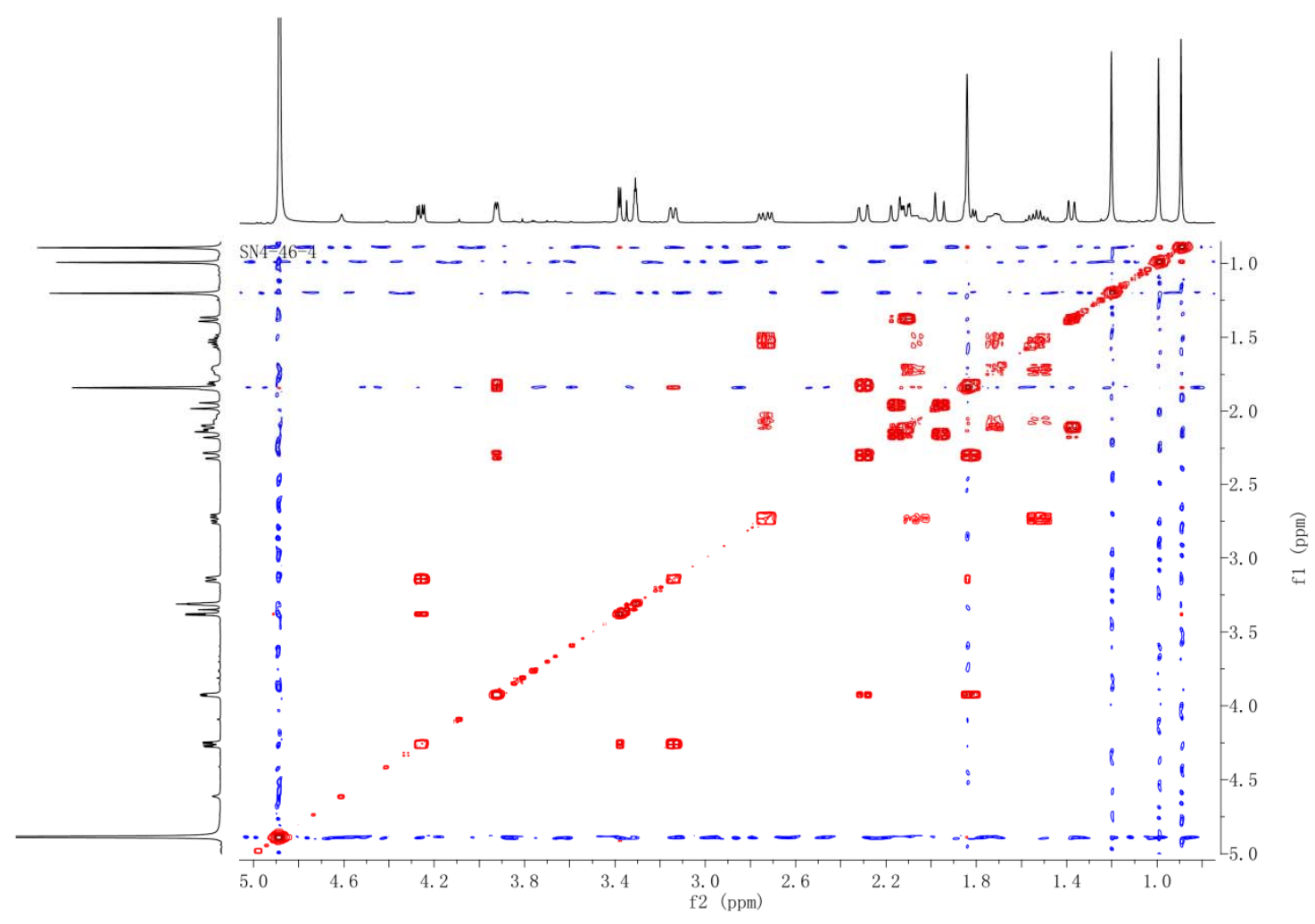

Figure S104. The ${ }^{1} \mathrm{H}^{-1} \mathrm{H}$ COSY (400 MHz, methanol- $d_{4}$ ) spectrum of compound $\mathbf{1 1}$

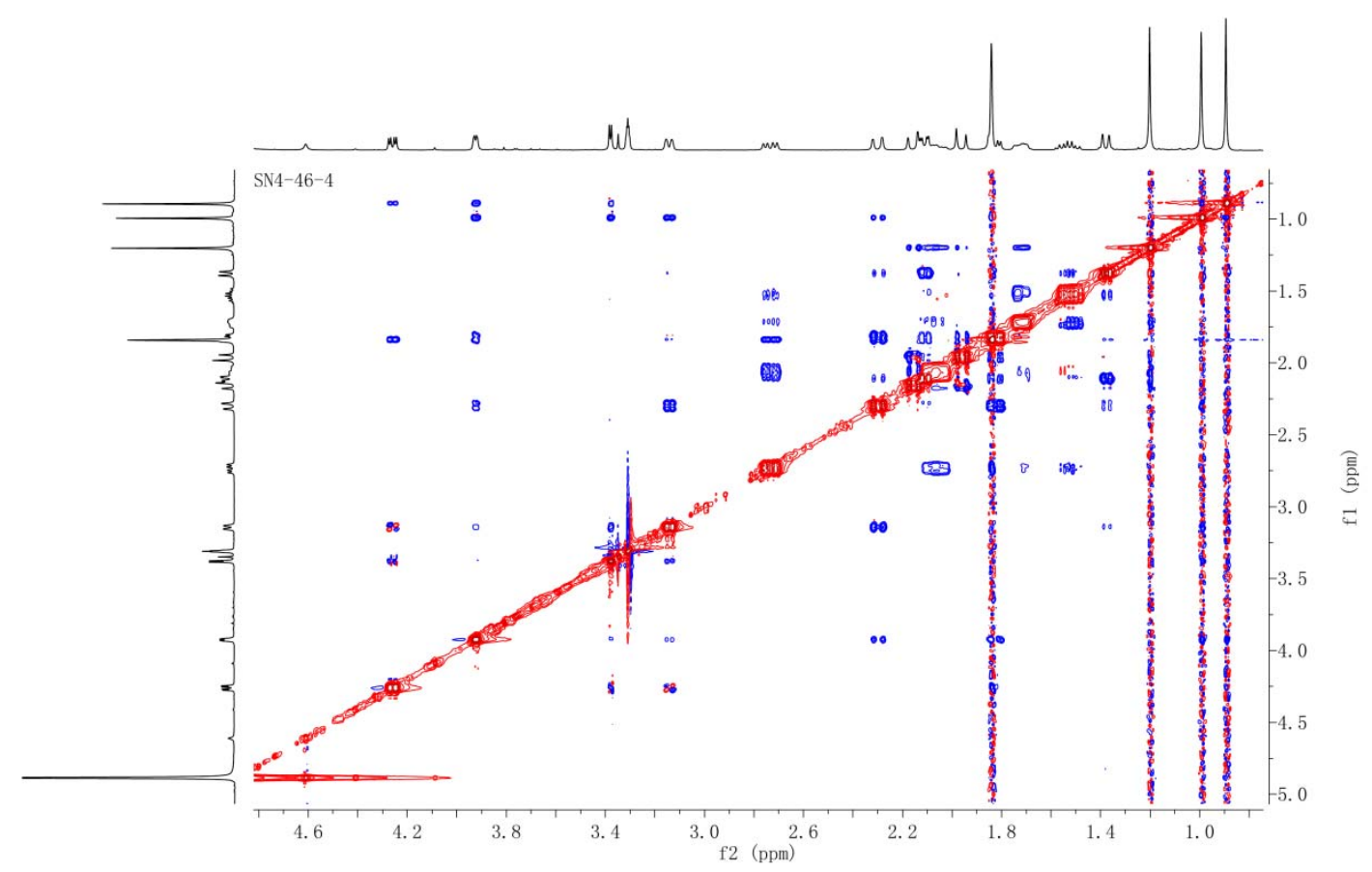

Figure S105. The NOESY (400 MHz, methanol- $d_{4}$ ) spectrum of compound 11 


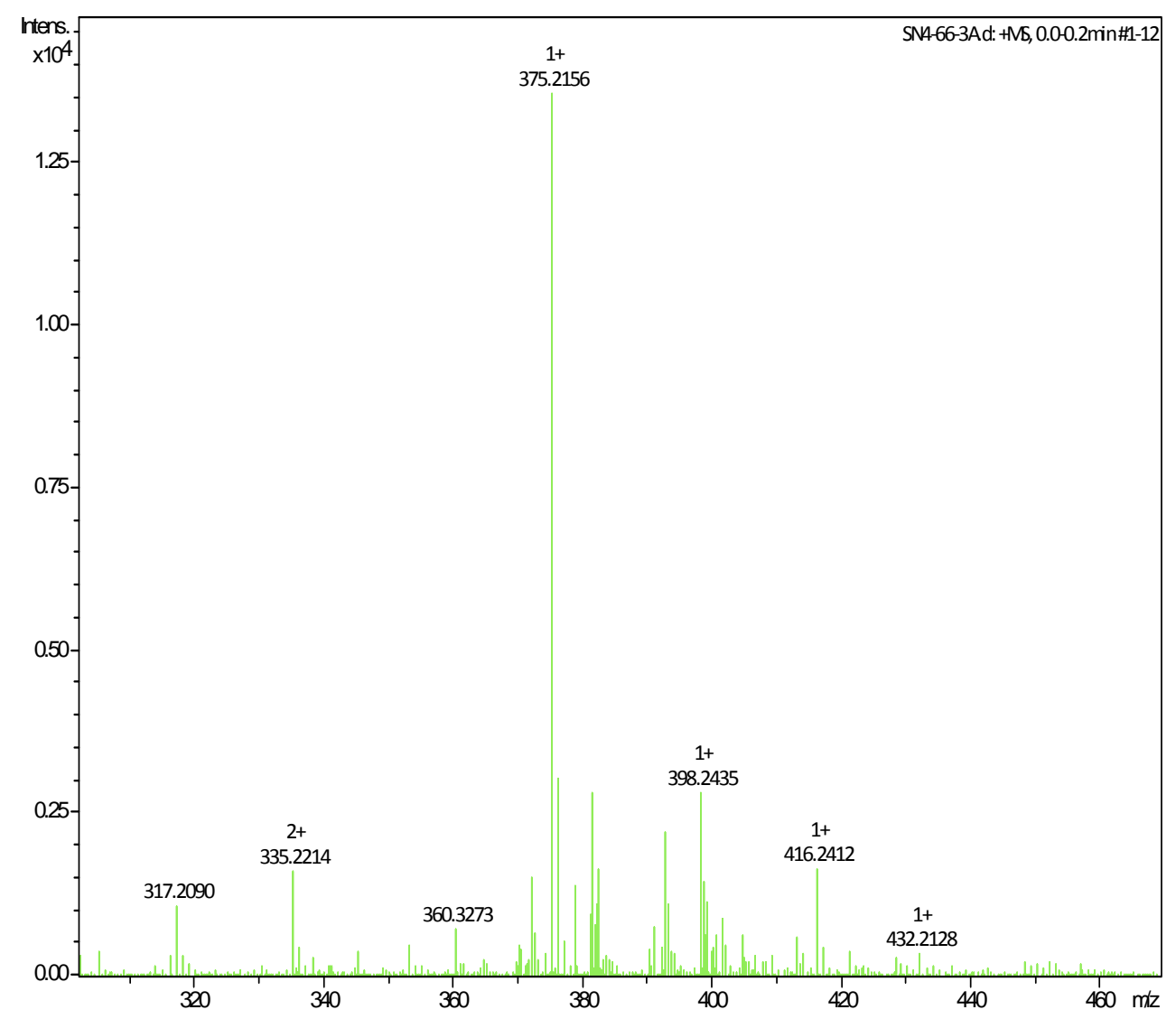

Figure S106. (+)-HRESIMS of compound 12

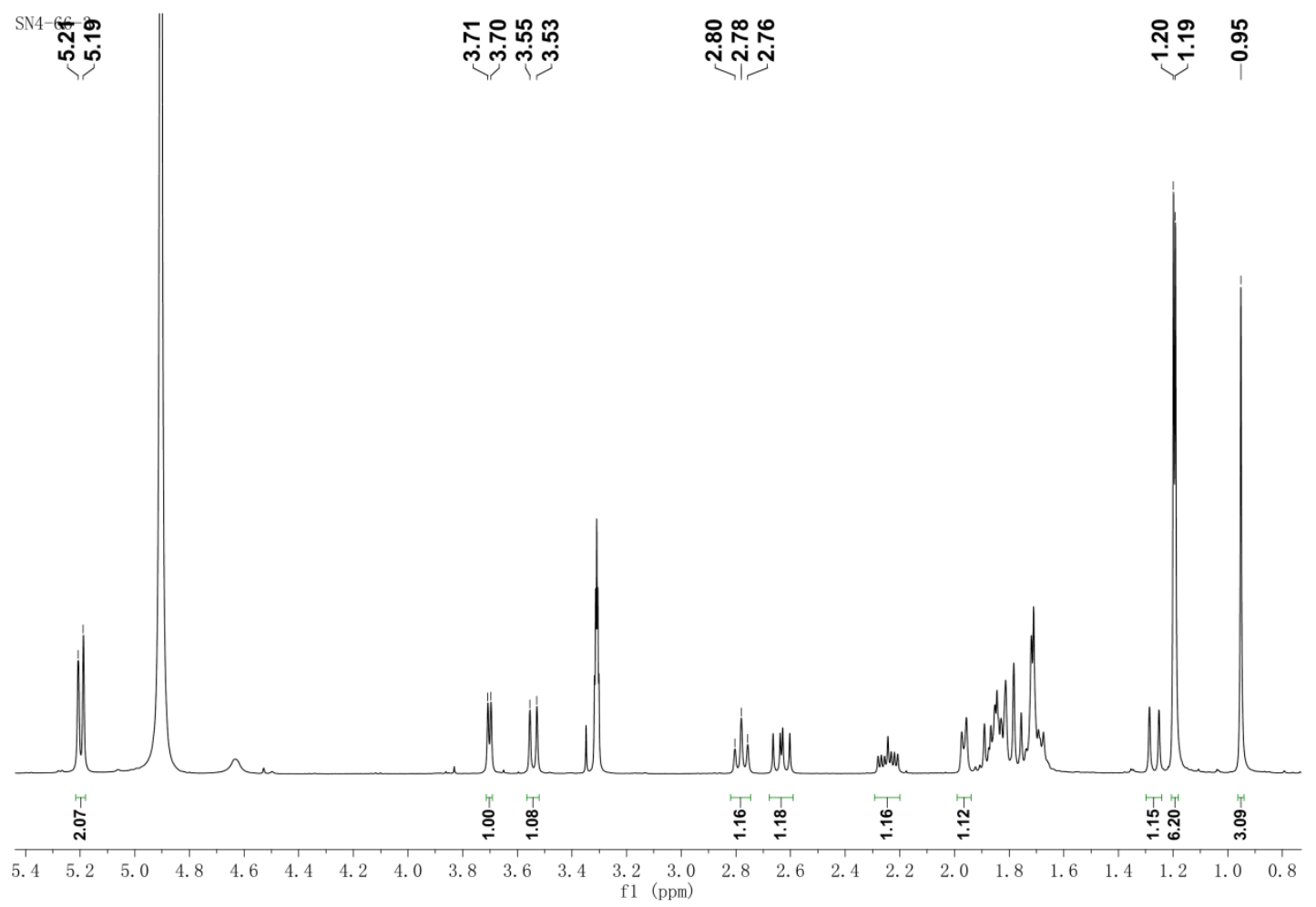

Figure S107. ${ }^{1} \mathrm{H}$ NMR (400 MHz, methanol- $\left.d_{4}\right)$ spectrum of compound 12 

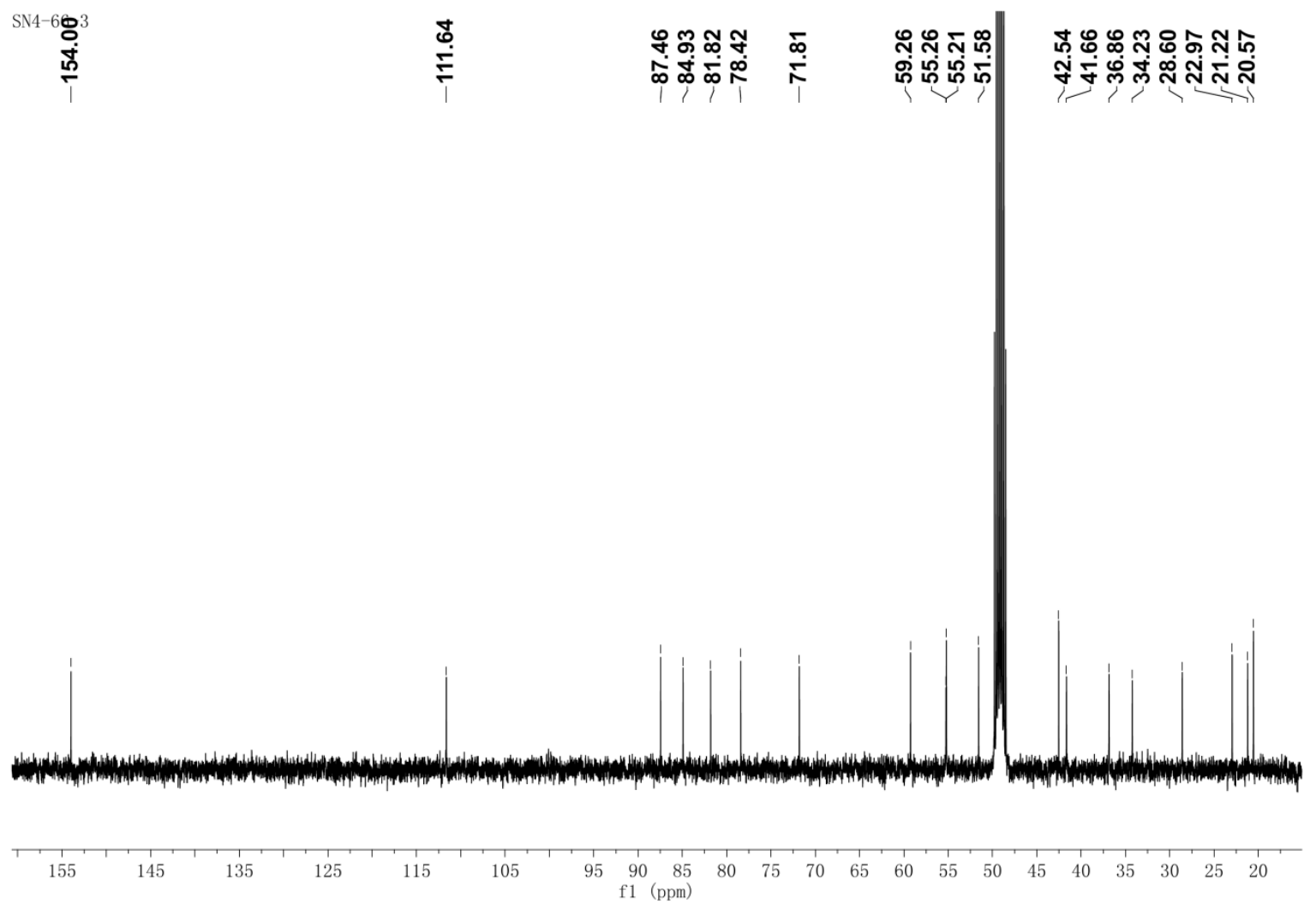

Figure S108. ${ }^{13} \mathrm{C}$ NMR (100 MHz, methanol- $\left.d_{4}\right)$ spectrum of compound 12

SN4-66 过
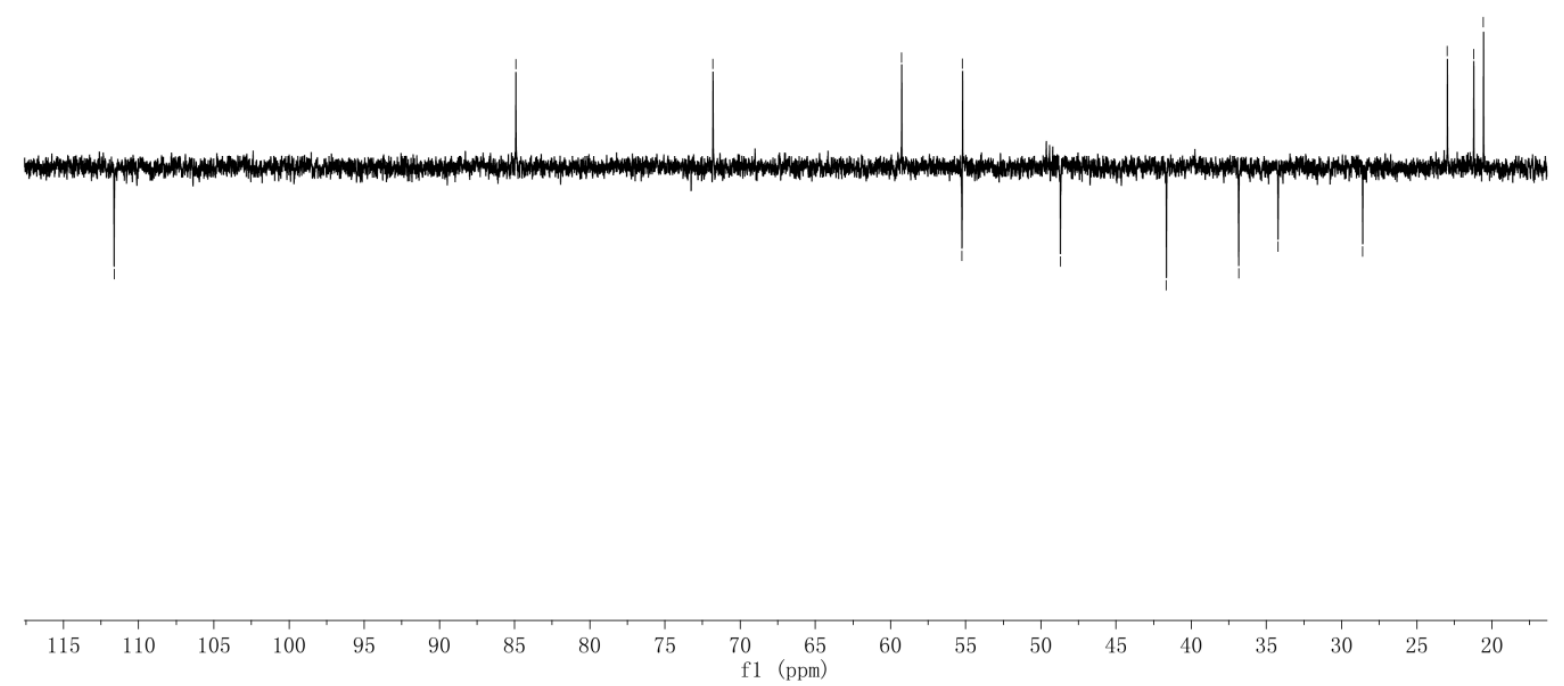

Figure S109. DEPT (100 MHz, methanol- $\left.d_{4}\right)$ spectrum of compound 12 


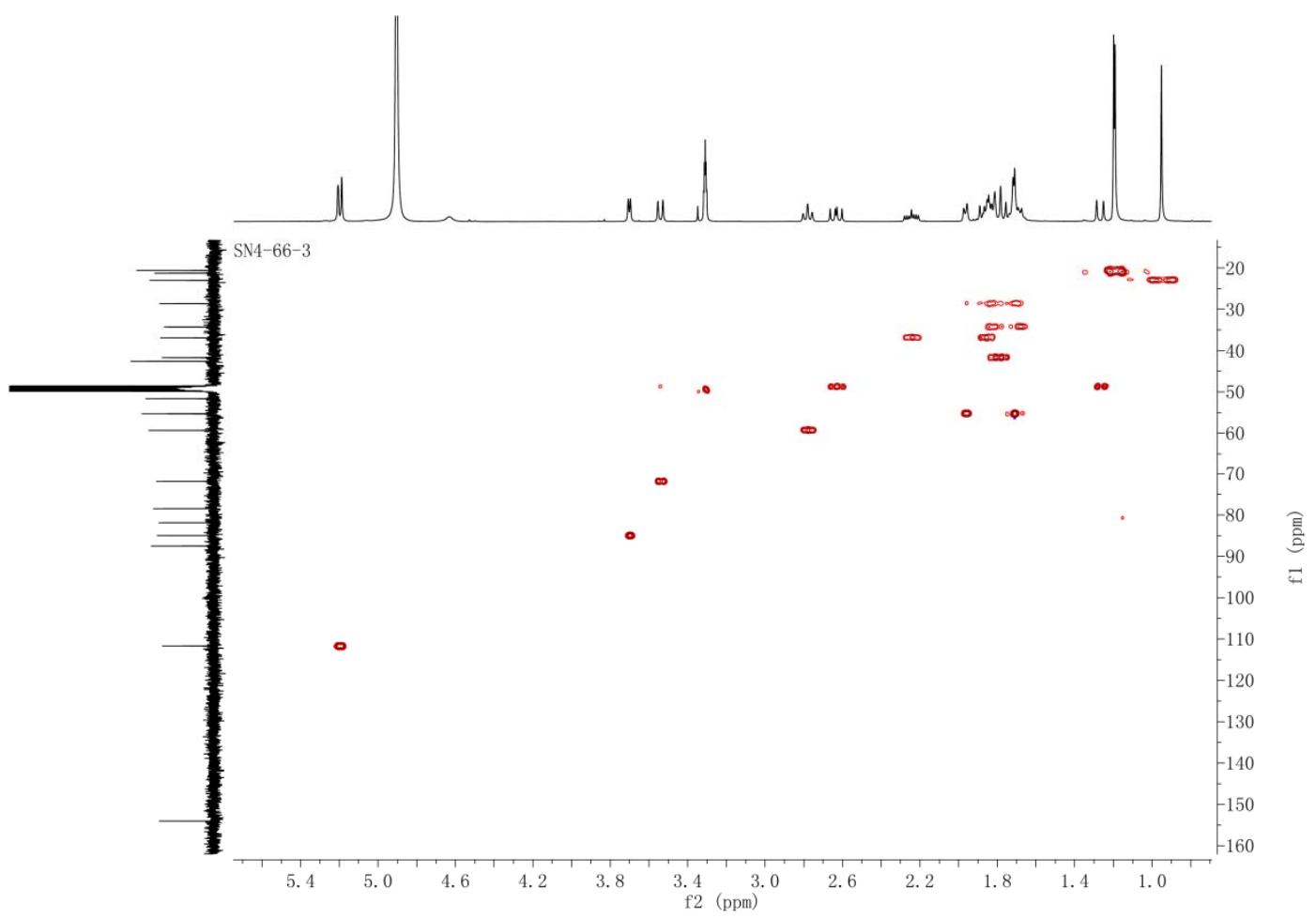

Figure S110. HSQC (methanol- $d_{4}$ ) spectrum of compound $12\left({ }^{1} \mathrm{H}: 400 \mathrm{MHz},{ }^{13} \mathrm{C}: 100 \mathrm{MHz}\right)$

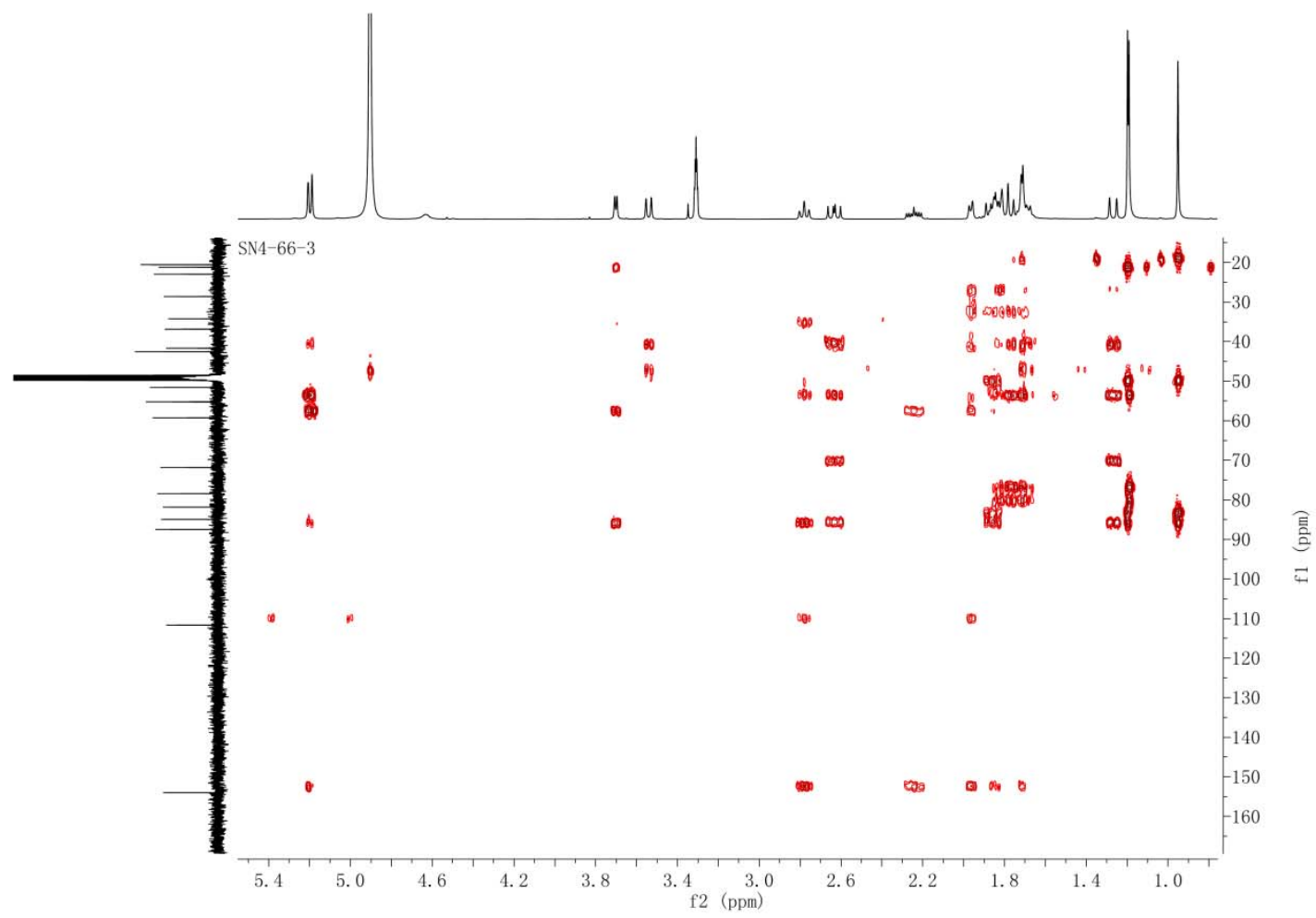

Figure S111. HMBC (methanol- $\left.d_{4}\right)$ spectrum of compound $12\left({ }^{1} \mathrm{H}: 400 \mathrm{MHz},{ }^{13} \mathrm{C}: 100 \mathrm{MHz}\right)$ 


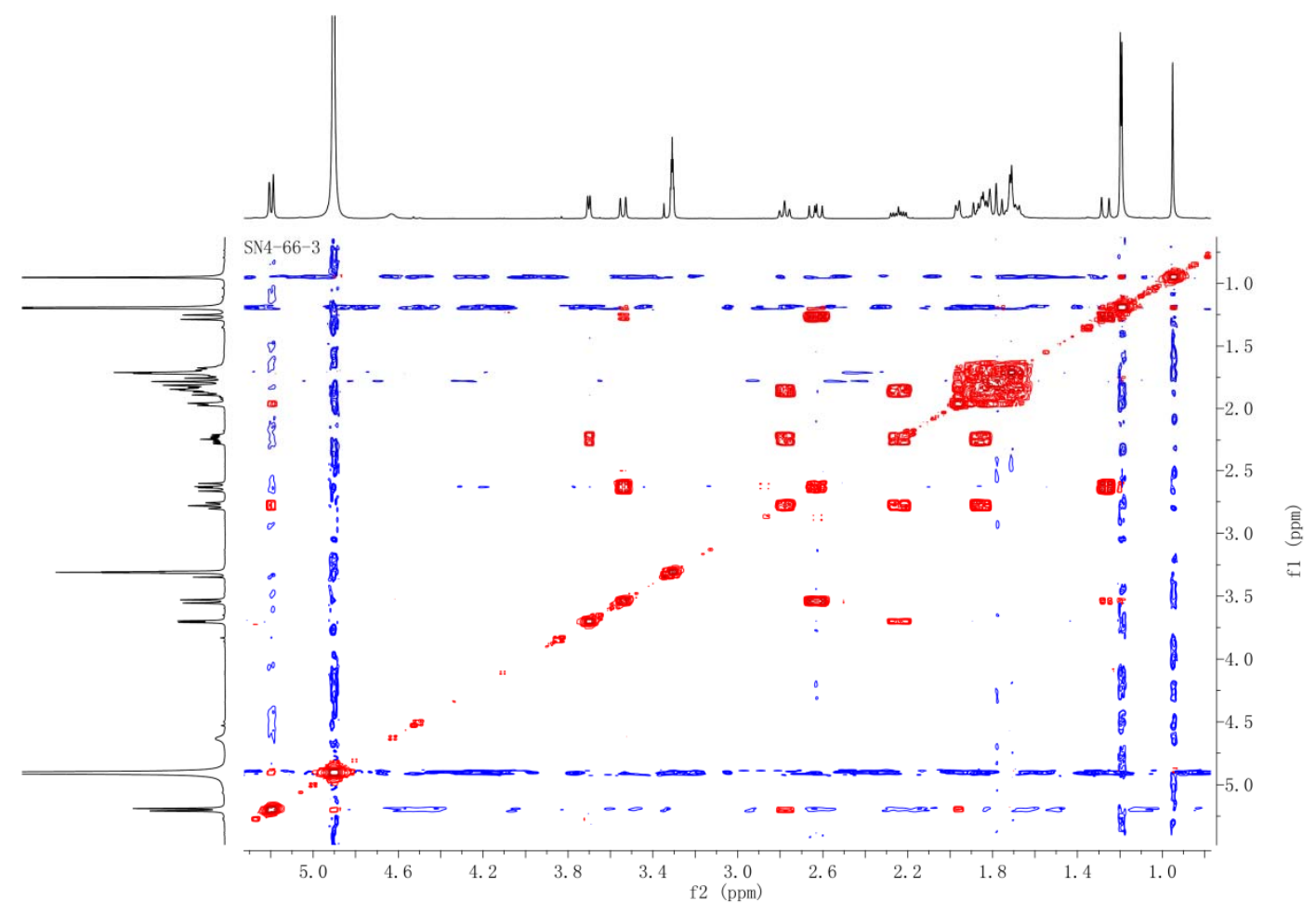

Figure S112. The ${ }^{1} \mathrm{H}^{-}{ }^{1} \mathrm{H}$ COSY (400 MHz, methanol- $d_{4}$ ) spectrum of compound 12

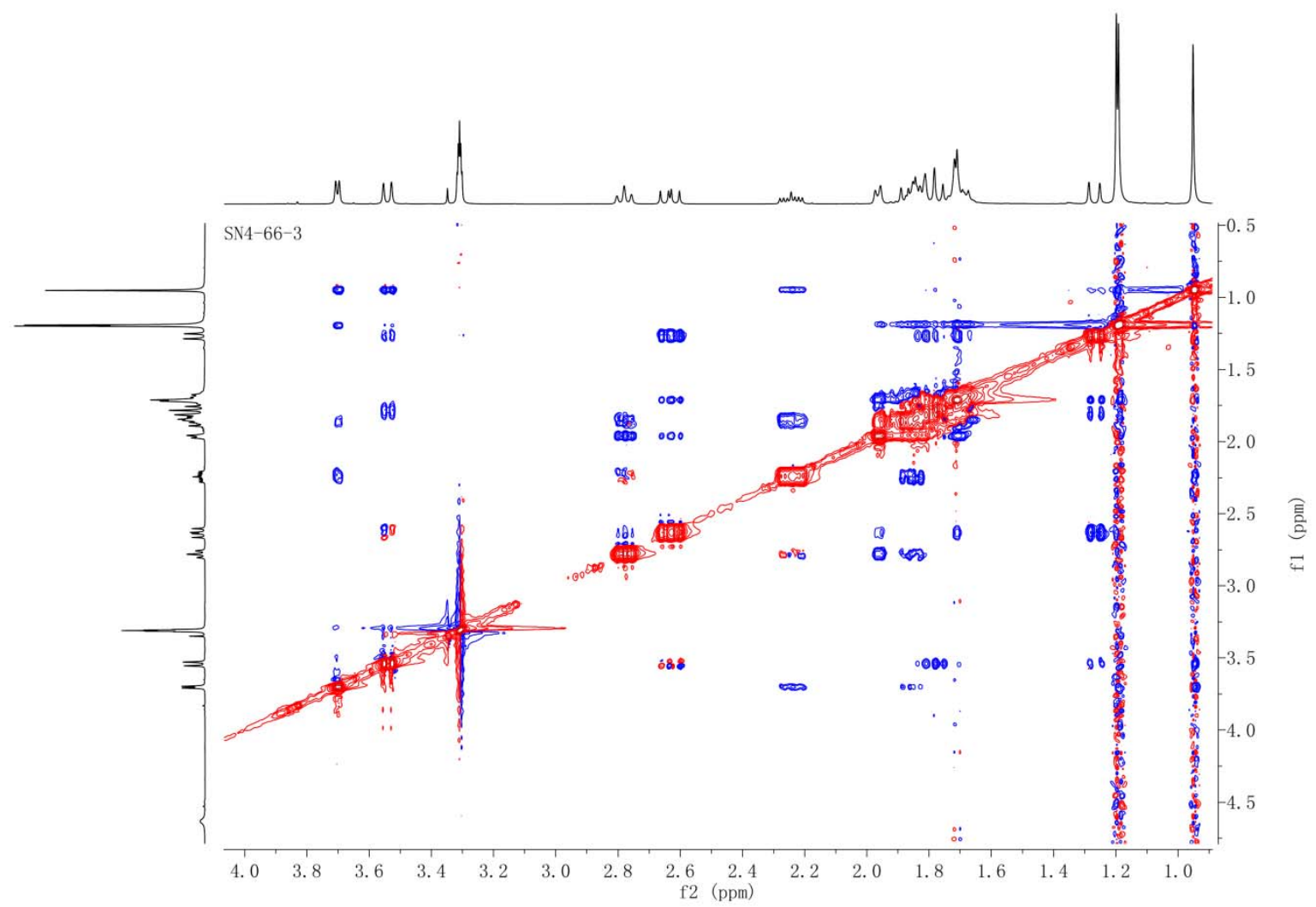

Figure S113. The NOESY (400 MHz, methanol- $d_{4}$ ) spectrum of compound 12 